\title{
Size-selective synthesis of nanometer- sized Palladium clusters and their hydrogen solvation behaviour
}

\author{
Dissertation \\ zur Erlangung des Doktorgrades \\ der mathematisch-naturwissenschatlichen Fakultäten \\ der Georg-August-Universität Göttingen \\ vorgelegt von \\ Mohammed A. M. Shtaya-Suleiman \\ aus Salfeet
}

Göttingen 2003 
D7

Referent: $\quad$ Prof. Dr. R. Kirchheim

Korreferent: $\quad$ Prof. Dr. H. Hofsäß

Tag der mündlichen Prüfung: Freitag, 25. Juli 2003 
الىىل حبة عرق ترقرقت من جبينه تعبا لاجلي والدي

الى من لهج لسانها بالدعاء لي واختطفها القدر مني روح والدتي الطاهره

الى حبيية القلب و رفيقة دربي وغربتي زوجتي

الى شعاع الامل ونور العين وامل المستقبل ابني الحبيب

الى من بحملون في عيونهم ذكريات الطفولة و الشباب اخواني واخواتي

الى من ضاقت السطور عن ذكرهم اصدقائي 


\section{Table of Contents}

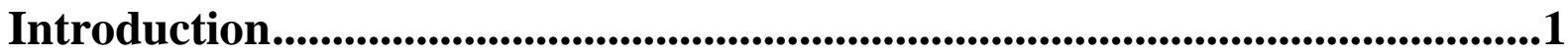

1. Hydrogen in Palladium ............................................................................................4

1.1 Thermodynamic basics ...............................................................................

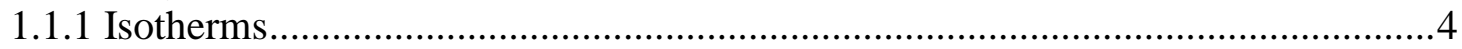

1.1.2 Hydrogen solubility in metals .........................................................................6

1.1.3 The Phase transition......................................................................................

1.1.4 Hysteresis ..............................................................................................

1.2 Hydrogen Metal Interaction ...................................................................................13

1.2.1 Physical and Chemical Adsorption.....................................................................13

1.2.2 Interaction with nanometer-sized Materials .........................................................15

2. Metal Clusters …...............................................................................................16

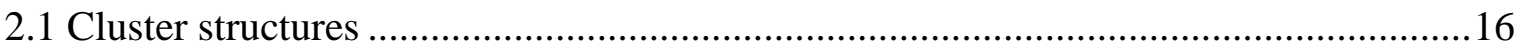

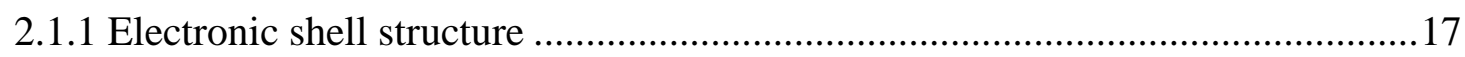

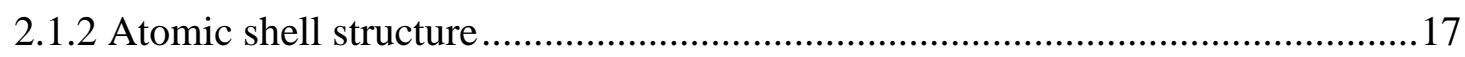

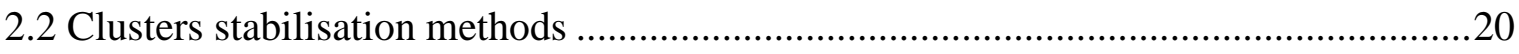

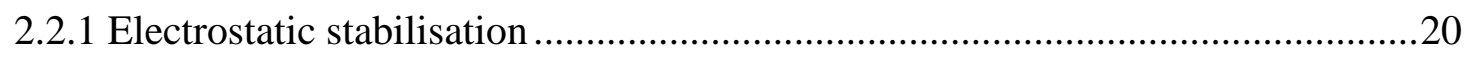

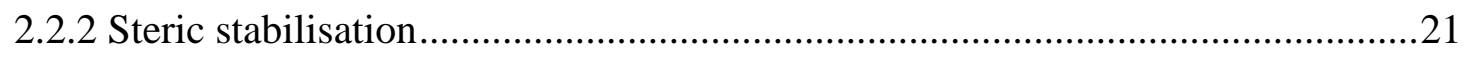

2.3 Clusters preparation methods .........................................................................23

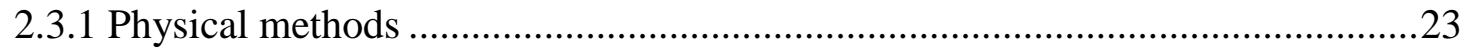

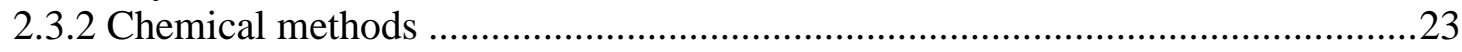

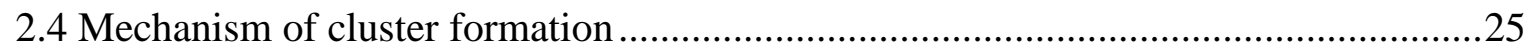

2.5 The Thermodynamics of Electrochemical Phase Formation .........................................27

3. Experimental Techniques and Methodology .............................................31

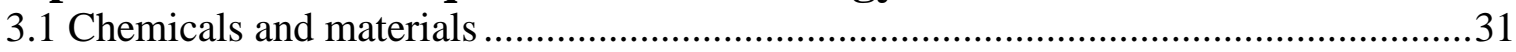

3.2 Electrochemical cluster preparation ………………………...................................

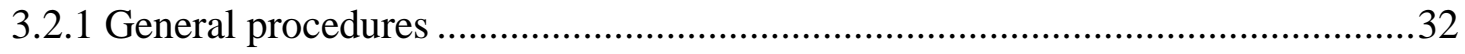

3.3 Experimental characterisation techniques ……………………………………......33

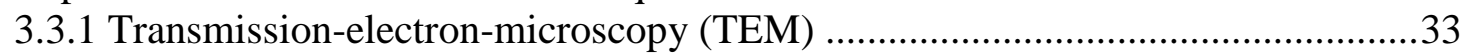

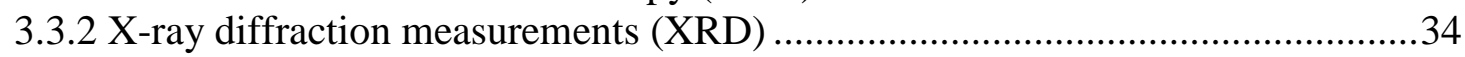

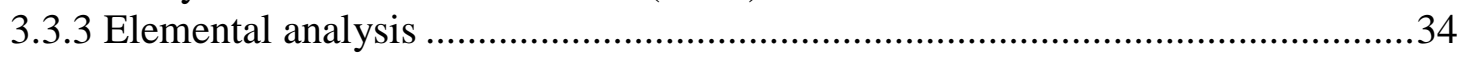

3.3.4 In-situ XRD during hydrogen loading unloading ................................................35

3.3.5 Gravimetric measurements .............................................................................36

4. Results: Pd Clusters Synthesis and Characterisation .......................................38

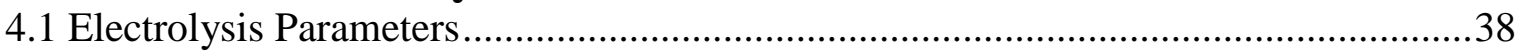

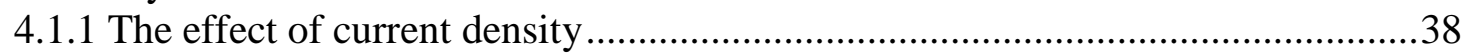

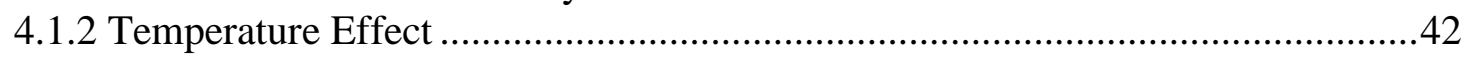

4.1.3 The Effect of the Distance between the Electrodes .............................................44

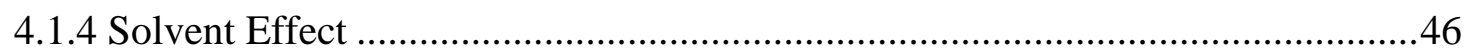

5. Discussion: Pd Clusters Preparation .............................................................49

5.1 Influence of the electrolysis parameters ..............................................................49

5.1.1 Current density effect ..................................................................................

5.1.2 Temperature effect.......................................................................................52

5.1.3 The effect of the distance between the electrodes $D_{E} \ldots \ldots \ldots \ldots \ldots \ldots \ldots \ldots \ldots \ldots \ldots \ldots \ldots \ldots \ldots . . . . . .53$

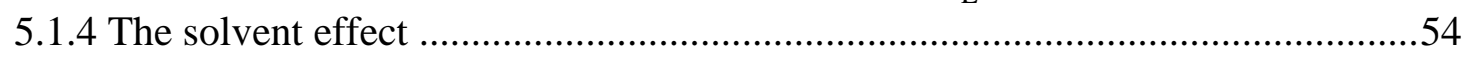

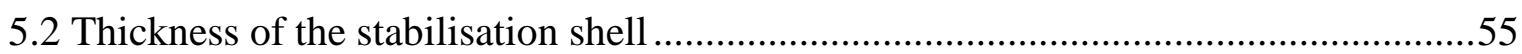

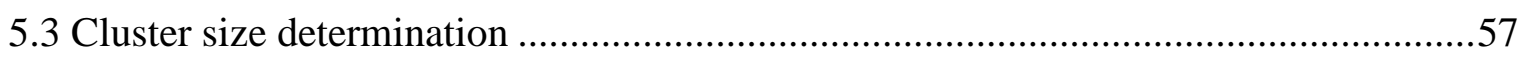

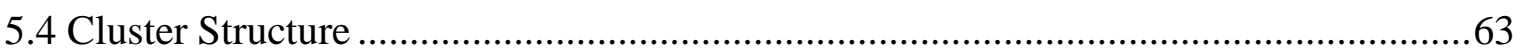

6. Results: Pd-H Clusters .........................................................................67 


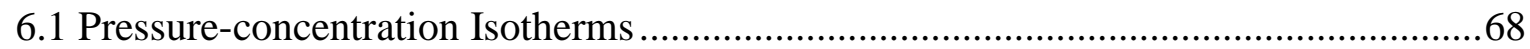

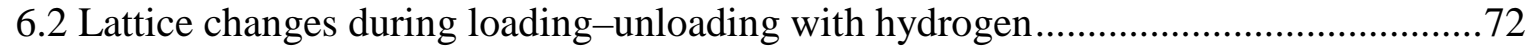

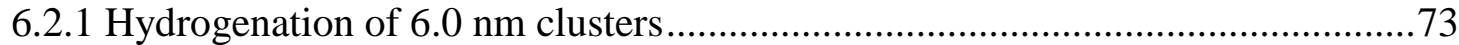

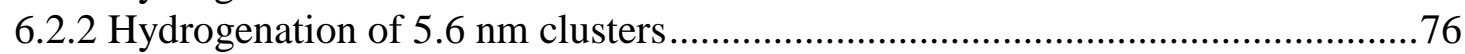

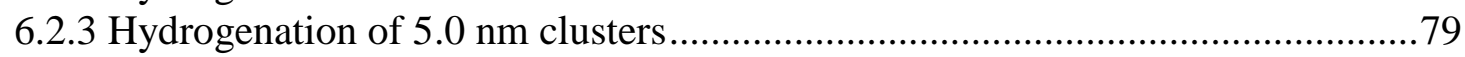

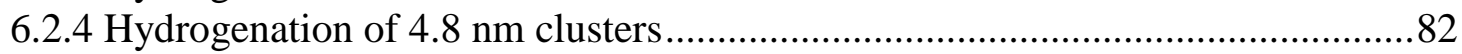

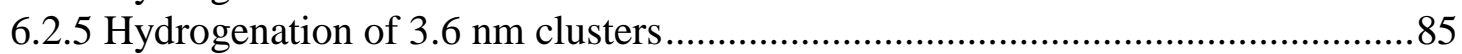

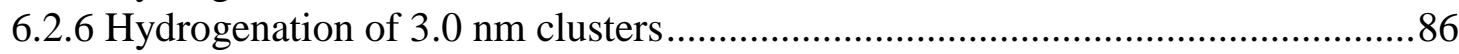

7. Discussion: Pd-H Clusters.........................................................................87

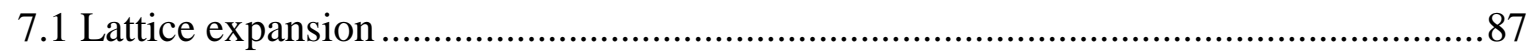

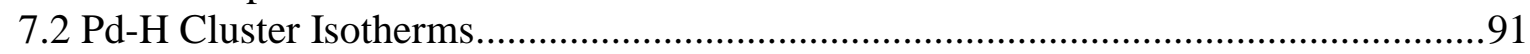

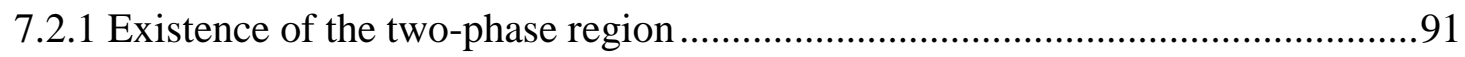

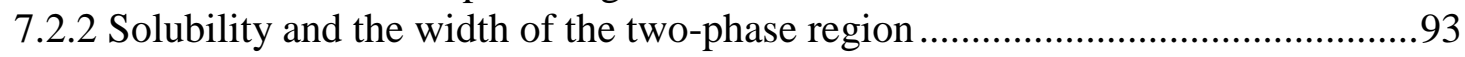

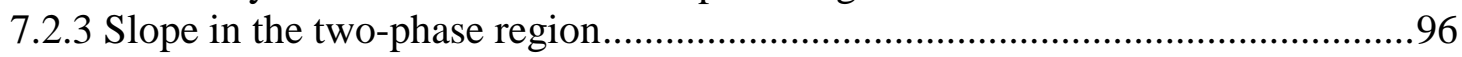

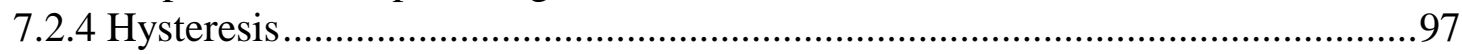

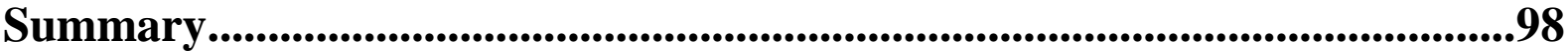

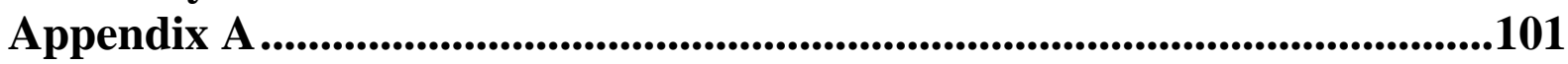

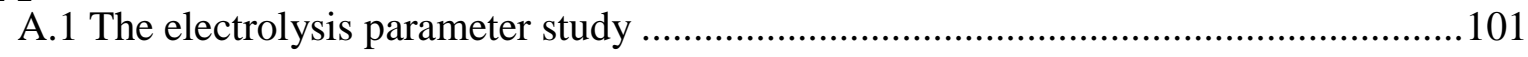

A.1.1 Electrolysis at different current density ........................................................101

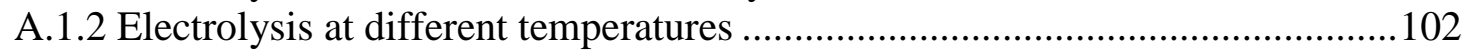

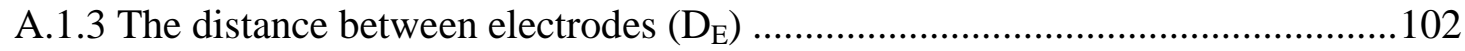

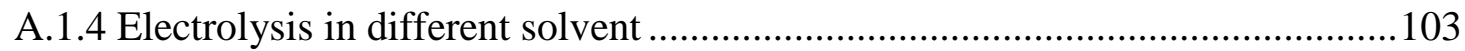

References....................................................................................................104 


\section{Introduction}

A silvery white metal named after the goddess of wisdom. It does not tarnish in air. Mixing it with gold produces the white gold used in jewelry. Closely related to platinum, it is used in dentistry, watchmaking, surgical instruments and electrical contacts. The metal is Palladium and it is most remarkable property, actually, is none of the above. Palladium can soaks up hydrogen like a sponge, that is, if you can imagine a sponge that soaks up hundreds of buckets of water. At room temperature and atmospheric pressure, Palladium can absorb up to 900 times it is own volume of hydrogen [1]. That means if you were to pump hydrogen into a bottle, it would take enormous pressure to store the same amount easily absorbed in a Palladium bed of the same volume.

Soon after the discovery of metal Palladium in 1803 by W.H. Wollaston it was observed that this metal could absorb large amounts of hydrogen. This was the starting point of an interesting area of research "hydrogen in metal" for both fundamental and technological aspects.

The Palladium hydrogen $(\mathrm{Pd}-\mathrm{H})$ system is the first of all metal hydrogen $(\mathrm{M}-\mathrm{H})$ systems that has attracted research activities, and been extensively investigated since the observation of Graham (1866) [2]. The broad interest is primly due to the relatively high hydrogen concentration for an elemental metal and the noble character of palladium.

With the progress in the preparation and the analysis of nanometer sized materials, such as nanocrystalline thin films and clusters, the behaviour of hydrogen in metallic system with reduced dimensions has become a very attractive research field. The physical and the chemical properties of nanometer sized materials, are often size dependent and are different to bulk properties [3-12].

For nanometer sized materials, the Pd-H system can be regarded as a model system. A nanocrystalline material was used to investigate the hydrogen palladium interaction in small grains. Mütschele and Kirchheim [4] found, that, in a nanocrystalline Pd-sample, there is an increase in the maximum $\alpha$-phase concentration, while the minimum $\alpha^{\prime}$-phase concentration decreases as compared to a polycrystalline sample. Similar results were obtained by other workers such as Natter et al. [5] and Stuhr et al. [6], in conformity with Mütschele and Kirchheim's interpretation, explaining the results in terms of the influence of grain boundaries which undergo no phase transition. Using neutron energy loss scattering, Stuhr et al. 
confirmed Kirchheim and Mütschele view that two types of hydrogen sites exist at low hydrogen concentrations: interstitial sites in the lattice and in the grain boundaries. The latter are occupied at a lower chemical potential and could therefore increase the $\alpha$-phase solubility. An increased solubility of hydrogen in the $\alpha$-phase and a narrowing of the miscibility gap in clusters were also observed [7-11], in good accordance with results on nanocrystalline materials. This behaviour was attributed to grain boundary-lattice sites that differ from bulk sites [4-6].

The preparation and properties of narrowly distributed nanometer sized metal colloids or metal clusters have been extensively studied because of the intense scientific interest in exploring the properties of small metal particles, and because of the anticipation in new technological applications [12]. They can be used as quantum dots for understanding the quantum size effects and for designing new optical and electronic materials [13-16]. The transition metal nanometer sized clusters also serve as a bridge between homogeneous and heterogeneous catalysis and provide new opportunities for catalysis [17]. They are not only active and selective catalysts for various organic reactions such as hydrogenation [18], hydrosylilation [19] and isomerisation, but also active catalysts for visible light-induced hydrogen evolution [20].

However, systematic studies on the hydrogenation behaviour of single-sized clusters are rare. Studying cluster in the size range of 2 to $6 \mathrm{~nm}$ is of particular interest because of the percentage of surface atoms varies strongly in this size range and the different structures that the cluster could have in this size range. Thus, it is of great importance to have single-sized clusters in performing such studies. Modification in the pressure-concentration $(p-x-T)$ isotherms found in nanometer sized Pd samples were attributed to the wide size distribution of the sample [21]. A slope in two-phase region of the $p-x$-T isotherms of $\mathrm{Pd}$ cluster samples found by Salomons et al. was explained by a broad size distribution $( \pm 10.7 \mathrm{~nm})$ of the clusters. To clarify if this wide size distribution really affects the isotherm in the described way, studies in single-size cluster are necessary. Another important aspect which should also be considered in studying clusters is the stabilisation method. Cluster syntheses involve the use of a stabilising agent which associates with the surface and prevent the nanoparticles from undesired aggregation. Stabilisation can be achieved by adhesion to a substrate or by embedding in a crystalline matrix [12]. The disadvantage of both methods is that the interfaces between the clusters and the substrates are not likely to participate in the catalytic reaction. Furthermore, large interface stresses may occur between the cluster and the substrate that are known to influence the clusters properties [22,23]. These disadvantages can be 
overcome by using elastically soft stabilisers such as polymers and surfactants [64]. Polymer stabilised clusters exhibit a slow kinetics of hydrogen absorption [8]. However, fast kinetics can be achieved by surfactant stabilised clusters that will be used in this work.

The aim of this work can be summarised in two main goals: First, preparation of different size of surfactant stabilised Pd clusters and studying the effect of the preparation parameters on the size and on the size distribution of the clusters. Second, studying the interaction of Pd-clusters with hydrogen from both kinetic and thermodynamic point of view.

This work will be divided into seven chapters. In chapter one a short overview on the thermodynamic basics of the $\mathrm{Pd}-\mathrm{H}$ system will be presented. Pressure-concentration isotherms and pressure-lattice parameter isotherms of bulk $\mathrm{Pd}$ will be provided. Hydrogen solubility in metals and the hydride formation including hyteresis will also be reviewed. This will be followed by discussing the hydrogen interaction with metal surface and with small grains.

In chapter two the metal clusters will be surveyed. First, results of the cluster structure based on the shell structure will be reviewed. This will be followed by discussing the stabilisation methods of clusters. An overview of the physical and chemical preparation methods of clusters will be given. In addition a mechanism and a thermodynamic approach of electrochemical phase formation will be presented.

Details about the experimental techniques and the experimental set-ups used in this work will be presented in chapter three.

In chapter four experimental results on the electrochemical preparation and characterisation of tetraoctylammonium bromide stabilised Pd clusters will be presented. The effect of the electrolysis parameter, such as the current density, the solvent, the temperature and the distance between the electrodes, on the size and the size distribution of the Pd cluster will be compiled. Determination of the cluster size and structure using different methods will be examined. Finally, the results of Pd clusters will be discussed in chapter five.

In chapter six results on the palladium-hydrogen clusters $(\mathrm{Pd}-\mathrm{H})$ with different cluster sizes will be presented including pressure-concentration isotherms for Pd clusters obtained by gravimetric measurements. Lattice expansion and phase transition of Pd clusters size with varying size will presented as they were obtained by in-situ X-ray diffraction (XRD) measurements during hydrogen loading-unloading cycles. The $\mathrm{Pd}-\mathrm{H}$ cluster results will be discussed in chapter seven. And details concerning the electrolysis parameters study will be provided in Appendix A. 


\section{Hydrogen in Palladium}

In the following the thermodynamic principles of the Palladium-Hydrogen system (Pd-H) will surveyed with detailled discussion of pressure-concentration isotherms and hydrogen solubility at low concentration. The phase transition and the hysteresis will be also viewed. This will be followed by discussing the hydrogen interaction with metal surfaces and nanometer-sized materials.

\subsection{Thermodynamic basics}

\subsubsection{Isotherms}

Hydrogen absorption in Pd metal, and most other metals, shows three distinguished parts in the pressure-concentration-isotherm $p-x-T$, Figure 1.1 (in logarithmic scale). The first part (I) is the solid solution ( $\alpha$-phase) where the pressure increases with increasing concentration according to Sieverts' law [24]. The second part (II) represents the two-phase region or the miscibility gap, between the saturated solid solution concentration and the concentration of the hydride phase $\left(\alpha^{\prime} \text {-phase }\right)^{1}$. The miscibility gap, at room temperature, is present between $\alpha$ $(0.008 \mathrm{H} / \mathrm{Pd})$ and $\alpha^{\prime}(0.607 \mathrm{H} / \mathrm{Pd})$. This part ideally exhibits no pressure dependence on the concentration (plateau). The third part (III) is metal hydride ( $\alpha^{\prime}$-phase). In this part, the pressure rises steeply with increasing concentration.

"Pressure-lattice parameter" isotherms can also be constructed. A correlation between changes in the lattice parameter and equilibrium hydrogen pressure was obtained by Owen and Jones in 1937. The study was performed on Pd powder at $100{ }^{\circ} \mathrm{C}$ without measuring the changes of hydrogen content. Plots of lattice parameter against the equilibrium pressure of hydrogen were clearly of similar form to those of pressure-concentration isotherms, see Figure 1.2.

1 The palladium hydride phase has an $f c c$ structure as the $\alpha$-phase and is designated as $\beta$-phase or $\alpha^{\prime}$-phase. In this work it will be designated as $\alpha^{\prime}$-phase. 


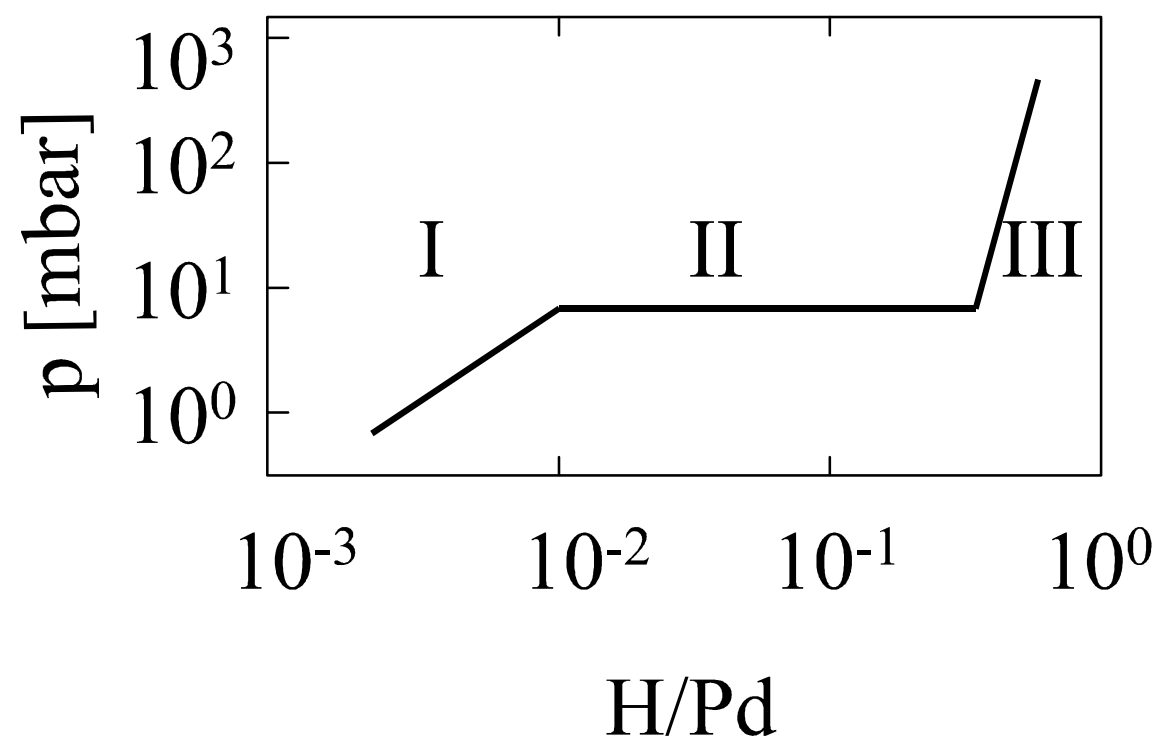

Figure 1. 1. Pressure-concentration-isotherm for the $\mathrm{Pd}-\mathrm{H}$ system at $20^{\circ} \mathrm{C}$ after [25].

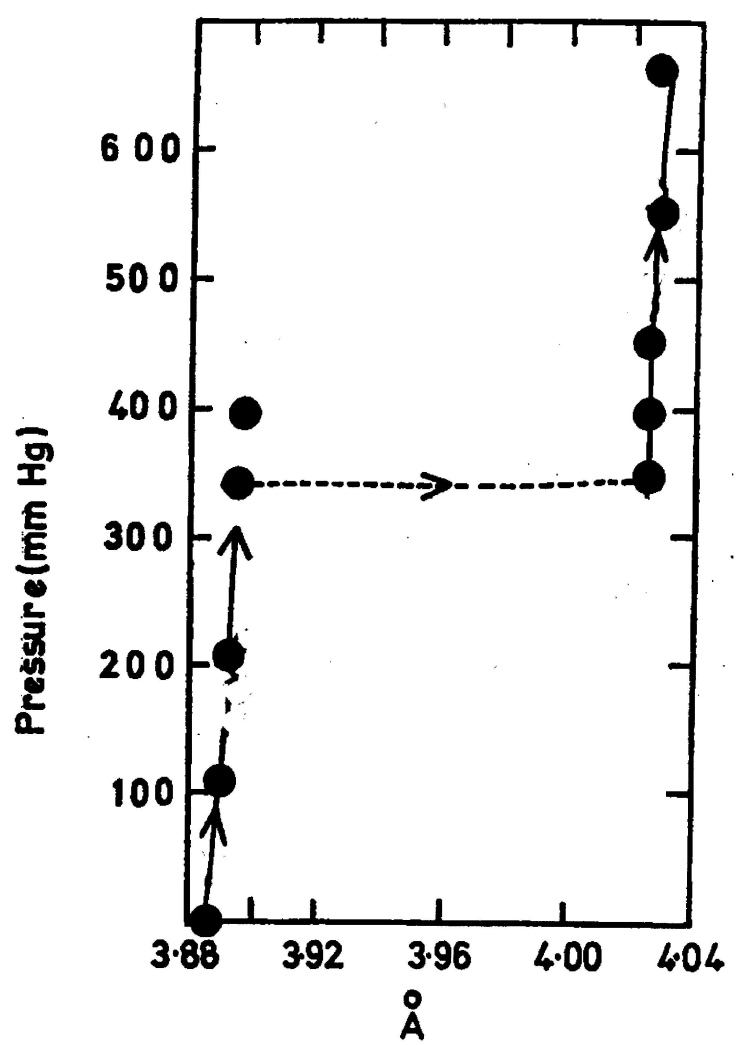

Figure 1. 2. Relationship between lattice constants and hydrogen pressure during absorption and desorption of hydrogen by $\mathrm{Pd}$ powder at $100^{\circ} \mathrm{C}$ after [ 26]. 


\subsubsection{Hydrogen solubility in metals}

The metal hydrogen system (M-H) with small hydrogen concentration in metal can be regarded as an ideal solution with no interaction between the solved particles. At constant temperature, one finds the following relationship between the outside hydrogen pressure $p$ and the hydrogen concentration $x$ in the metal:

$$
x=\frac{1}{k_{s}} \sqrt{p}
$$

This relation is called Sieverts' law where, $k_{s}$ is the Sieverts constant which depends on the temperature and $x$ is the ratio of the dissolved hydrogen atoms $\mathrm{H}$ to the number of metal atoms $\mathrm{M}, x=[\mathrm{H}] /[\mathrm{M}]$.

The position of the adsorbed $\mathrm{H}$ atoms in the metal lattice can be determined by neutron diffraction or by inelastic scattering. Measurements show that the $\mathrm{H}$ atom occupies only one of two types of interstitial sites: octahedral $\left(O_{h}\right)$ and tetrahedral $\left(T_{h}\right)$. For a face-centered-cubic $f c c$ lattice structure, e.g Pd metal, $\mathrm{H}$ occupies the $O_{h}$-sites. The number of $O_{h}$-sites are equal to the number of the Pd atoms.

At constant temperature and pressure, a thermodynamic equilibrium is established between the solid solution (the $\alpha$-phase) and the hydrogen gas:

$$
M+\frac{1}{2} x H_{2} \Leftrightarrow M H_{x}
$$

At equilibrium the chemical potential of the hydrogen gas per molecule and the chemical potential of the hydrogen per atom dissolved in the metal are equal [27]:

$$
\frac{1}{2} \mu_{H_{2}}^{g}=\mu_{H}^{M}
$$

At low hydrogen pressure the hydrogen gas can be treated as an ideal gas. The chemical potential of an ideal gas can be written as:

$$
\mu_{H_{2}}^{g}=R T \ln \frac{P_{H_{2}}}{P_{o}}+\mu_{H_{2}}^{o}
$$


or

$$
\mu_{H_{2}}^{g}=k_{B} \cdot T \cdot \ln \frac{p}{p_{o}(T)}-E_{d}
$$

with,

$$
p_{o}(T)=\frac{\left(4 \pi M k_{B} T\right)^{3 / 2} k T \cdot 8 \pi^{2} I_{r} k_{B} T}{h^{5}}
$$

where $R$ is the gas constant, $P_{o}$ is the standard pressure (1atm), $\mu_{H_{2}}^{o}$ is the standard values of the chemical potential for $P=P_{o}, M$ is the mass of an $\mathrm{H}$ atom, $I_{r}$ is the moment of inertia, $E_{d}$ is the heat of dissociation of an $\mathrm{H}_{2}$ molecule $(4.74 \mathrm{eV}), k_{B}$ is the Boltzmanns constant, and $h$ is the Planck's constant [28].

According to Wagner [29] and Brodowsky [30] the chemical potential of $\mathrm{H}$ atoms dissolved in Pd metal can be set to:

$$
\mu_{H}^{M}=\mu_{H}^{o}+R T \cdot \ln \frac{x}{1-x}+\Delta \mu_{H}
$$

where;

$\mu_{H}^{o} \quad$ is the standard potential. $R T \cdot \ln \frac{x}{1-x}$ is the configurational term for the ideal statistic distribution of the hydrogen
atoms among the $O_{h}$-sites.

$\Delta \mu_{H}=\Delta \mu_{H^{+}}+\Delta \mu_{e} \quad$ stand for the deviation from ideal solution behaviour.

\subsubsection{The Phase transition}

Considering a very low hydrogen concentration in the metal lattice. In this case as shown in Fig.1.1, part (I), there is a strong $\mathrm{M}-\mathrm{H}$ interaction between the $\mathrm{H}$ atoms and the metal lattice and a negligibly weak $\mathrm{H}-\mathrm{H}$ interaction between the individual $\mathrm{H}$ atoms.

As the $\mathrm{H}$ concentration in the metal increases, the lattice expands. In this case, part (II) Fig 1.1, the $\mathrm{H}-\mathrm{H}$ interaction between the individual $\mathrm{H}$ atoms is no longer negligible. This 
corresponds to concentration-dependent [31], positive exchange energy of regular solution model [32], if $O_{h}$-sites and the $\mathrm{H}$ atoms are regarded as binary mixture.

Similarly, a miscibility gap (two-phase region) exists at a temperature lower than the critical temperature $T_{c}$. Within the concentration range of the miscibility gap both phases $\alpha$ and $\alpha^{\prime}$ exists. In this region the chemical potential of $\mathrm{H}$ stays constant. The $\mathrm{H}$ pressure remains constant due to this equilibrium condition.

Increasing the $\mathrm{H}$ concentration leads to an increase of the portion of the $\alpha^{\prime}$-phase. When the electronic density of states (DOS) does not allow further growth of the $\alpha^{\prime}$-phase with the same chemical potential, the $p-x-T$ isotherm turn-up strongly as shown in part (III) Fig 1.1.

The method of primary importance for determining the phase limits in the $\mathrm{Pd}-\mathrm{H}$ system is the measurement of pressure-concentration isotherms. The hydrogen content of the solid under the actual hydrogen pressure used to be determined by means of gas volumetry, gravimetric methods, or by measuring electric resistivity which had been calibrated with respect to $\mathrm{H}$ concentration. Using bulk Pd the gas-solid equilibrium is difficult to obtain at room temperature. This is due to the impurity layers at the surface inhibiting the dissociation of the impinging $\mathrm{H}_{2}$ molecules into atoms [33], therefore the early investigations at low temperatures were performed on Pd black. The kinetic was improved due to the large surface area of the Pd black. Since clusters have large surface area H-loading from the gas phase at room temperature is possible as we will see in this work.

Another method to measure at low temperatures is the "hydrogen transfer catalysts". This application extended the temperature range of measurements of bulk $\mathrm{Pd}$ to below $0{ }^{\circ} \mathrm{C}$. Example of transfer catalysis are finely dispersed uranium hydride $\left(\mathrm{UH}_{3}\right)$ or copper powder [34].

A valuable technique which has been used for loading Pd is electrochemical charging [35, 36,4]. The hydrogen uptake or loss by the Pd electrode is obtained by Faraday's law or from the electrochemical potential which is a direct measurement of the chemical potential of hydrogen in the sample. 
The plateau pressure in the two-phase region increase with temperature according to [33]:

$$
\ln p=-\frac{\Delta \mathrm{H}}{R T}+\frac{\Delta S^{o}}{R}
$$

with $\Delta \mathrm{H}=41.1 \mathrm{~kJ} \cdot \mathrm{mol}^{-1}$ of $\mathrm{H}_{2} ; \Delta S^{o}=97.8 \mathrm{~J} \cdot \mathrm{mol}^{-1} \cdot \mathrm{K}^{-1}$ of $\mathrm{H}_{2}$ at room temperature.

The $\alpha^{\prime}$-phase has a bigger lattice parameter than the $\alpha$-phase. At room temperature the best values of the lattice constant for pure Pd and for the coexisting $\alpha$ and $\alpha$-phases $\left(c=\alpha_{\max }=\right.$ 0.008 , and $\left.c=\alpha^{\prime} \min =0.607\right)$ are, respectively, [33]

$$
a(0)=3.890 \stackrel{o}{\mathrm{~A}}, \quad a\left(\alpha_{\text {max }}\right)=3.894 \stackrel{o}{\mathrm{~A},} \quad a\left(\alpha_{\text {min }}^{\prime}\right)=4.025 \stackrel{o}{\mathrm{~A}}
$$

Which makes it possible, by performing in-situ X-ray diffraction measurements during $\mathrm{H}$ loading-unloading, to monitor the phase transition in different Pd clusters samples, as will be shown in chapter 6 in this work.

\subsubsection{Hysteresis}

In the $p-x$ - $T$ diagram, the plateau pressure for the absorption $p_{a b}$ curve is usually higher than the one for desorption $p_{a d}$. This difference in the absorption-desorption pressure is called hysteresis, Figure 1.3.

$$
\text { Hysteresis }=\ln \left(\frac{p_{a b}}{p_{d e s}}\right)
$$

Hysteresis is not a unique materials property and is dependent on the sample's history and the test procedure used, and it is usually referred to as a result of non-thermodynamic measurements. Hysteresis is usually larger when determined by the dynamic (continuous) method as opposed to the static (point by point) method. Dynamic methods are an example of nonequilibrium measurements which leads to an increase in the absorption pressure and a decrease in the adsorption pressure resulting in enlargement in the hysteresis. 
Hysteresis is a phenomena which should not be only attributed to non-thermodynamic effect. The hydride formation is not completely a reversible process. Hydrogen expands elastically the metal lattice during absorption and the lattice relaxed during the adsorption. Although this is a reversible process the elastic contribution during the hydride formation, as a result of the phase formation, leads to the hysteresis.

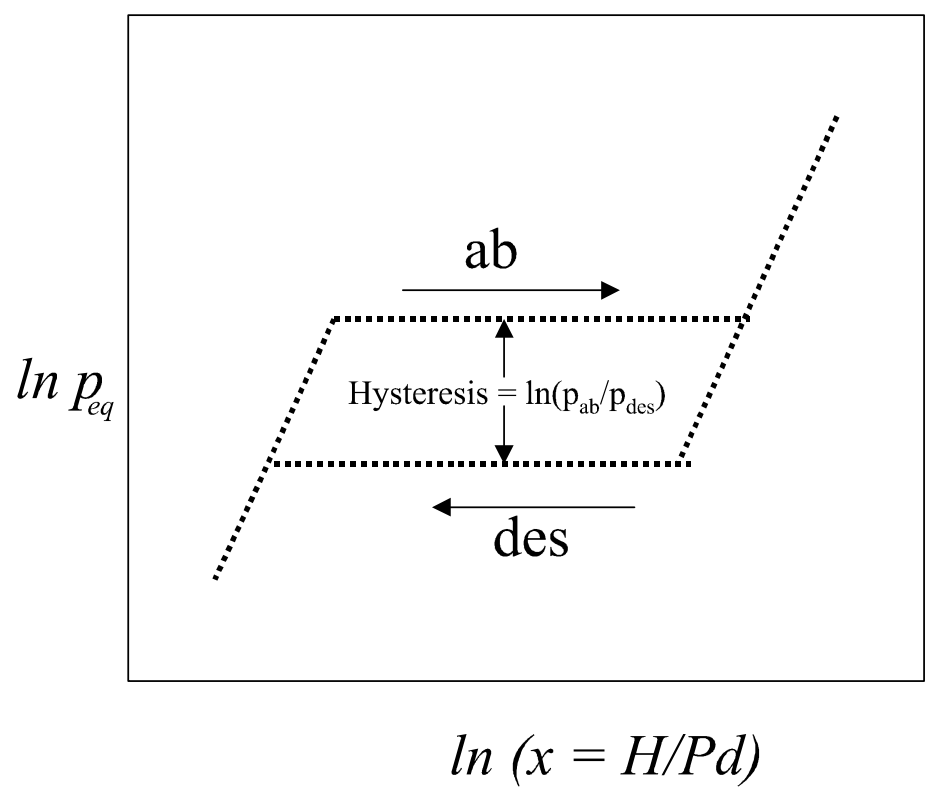

Figure 1. 3. Hypothetical pressure-concentration isotherm showing a hysteresis.

At the boundaries between two phases with different lattice parameter elastic stress will build up generating defects and dislocations. Thus there are two cases conceivable: coherent state and incoherent state. In the coherent case, the stress caused by the $\mathrm{H}$ atoms depends on the $\mathrm{H}$ concentration. The $\mathrm{H}$ concentration is non-homogeneously distributed over the sample resulting in stress-free condition on the sample surface. In this case the shape of the sample will affect the phases distribution. In the incoherent case, the stress is relaxed in the boundaries between the phases, accordingly, there is no sample shape dependence of the phases distribution. This case is the most common one in the M-H systems.

Flanagen et al. [37] and Makenas et al. [38] explained the existence of a hysteresis in bulk metal in terms of misfit dislocations. According to Brook's criterion [39] no misfit dislocations are expected to appear in Pd for hydride-precipitates that are smaller than $4 \mathrm{~nm}$. 
Brook estimated the critical size of precipitates in bulk material. In clusters even higher critical size is expected. Therefore the formation of misfit dislocation is impossible in clusters. Rather an elastic strain is expected. The explanation of a hyteresis must be different in the clusters case.

Based on the theory of Cahn and Larché [40], Schwarz and Khachaturyan [41] showed that a thermodynamic treatment of an open two-phase system, like the hydrogen-metal system, with coherent interfaces, already leads to the existence of a hysteresis. This thermodynamic approach leads to a multiplicity of effects which do not arise in the classical Gibbs treatment of two-phase system. For example, the total free energy of the coherent system depends nonlinearly on the volume fraction of the coexisting phases [42]. The strain energy depends on the spatial pattern (microstructure) formed by the coherent domains of the coexisting phases and therefore the microstructure becomes a thermodynamic parameter [42]. For such a system, the conventional common tangent construction of the additive Gibbs thermodynamic used to find the equilibrium composition of the coexisting phases ceases to be valid.

The graphical solution of this approach is shown in Figure 1.4. The stability limit of the phase is determined by common tangent construction applied to the chemical free energy curves. The corresponding chemical potential are taken from the total energy curves which includes the strain contributions. The characteristic relation between the absorption and the desorption pressure $p_{a b}$ and $p_{d e s}$ is:

$$
\ln \left(\frac{p_{a b}}{p_{d e s}}\right)=\frac{4 \Omega G_{s} \frac{1+v}{1-v} \varepsilon_{o}^{2}\left(c_{\alpha^{\prime}}-c_{\alpha}\right)}{k T}
$$

where $\Omega=2.607^{3}$ the volume of one hydrogen atom in $\mathrm{Pd}$, the shear modulus of $\mathrm{Pd} G_{s}=$ $47.4 \times 10^{9} \mathrm{~Pa}$, the Poisson number $v=0.385$, the change in the lattice constant $\varepsilon_{o}=0.063$, and the boundaries of the two-phase region $\mathrm{c}_{\alpha}=0.008, \mathrm{c}_{\alpha^{\prime}}=0.607$.

This thermodynamic approach was found to be in good agreement with the experimental results obtained from Pd-clusters by our group [7]. Generally, the existence of a hysteresis always implies the transition between two phases. 


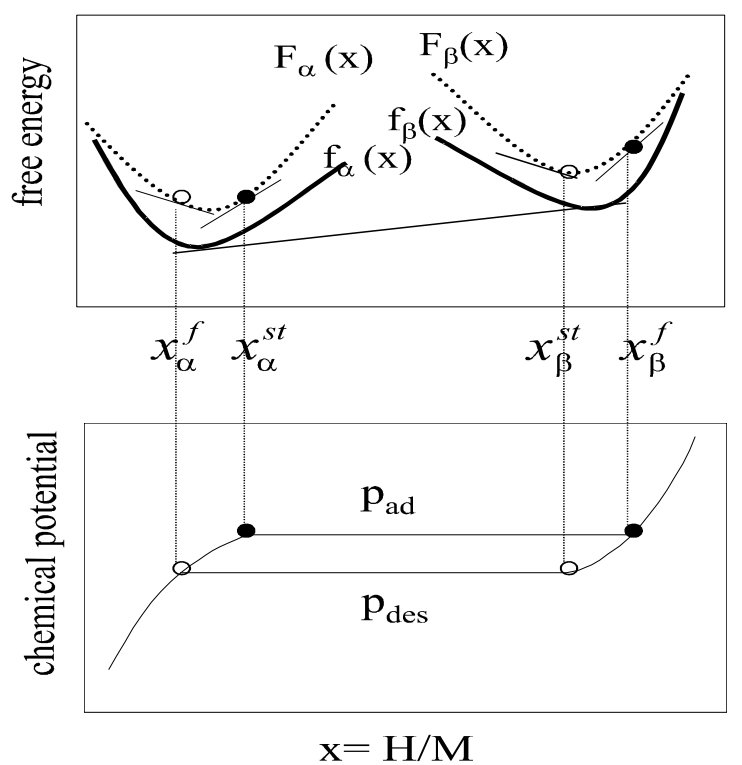

Figure 1. 4. Top: schematic free energy curves as a function of composition of interstitial atoms for the homogeneous $\alpha$ and $\beta$ phases of a two-phase system with coherent interfaces. The solid curve $f_{\alpha}(x)$ and $f_{\beta}(x)$ represent the chemical contribution to the free energies. Bottom: chemical potential of the interstitial hydrogen atoms in the gas phase (depends on the logarithm of gas pressure according to eqn. 1.3) as a function of the composition H-concentration after [41]. 


\subsection{Hydrogen Metal Interaction}

Coming to small size systems one has to take the surfaces into account. In this section the hydrogen metal interaction with metal surface using a one-dimensional energy curve will be discussed. This will be followed by examining the hydrogen interaction with nanometer sized materials. Because of similarities between clusters and nanocrystalline materials results obtained from nanocrystalline materials will be also reviewed in the following section.

\subsubsection{Physical and Chemical Adsorption}

The adsorption of $\mathrm{H}$ can be described using a simplified one-dimensional potential energy curve for an $\mathrm{H}_{2}$ molecule and two $\mathrm{H}$ atoms on a metal surface [43], Fig 1.5. A $\mathrm{H}_{2}$ molecule approaching the metal surface can be physisorbed by the attractive van der Waals interaction between the $\mathrm{H}_{2}$ and the metal. At the interface between the $\mathrm{H}_{2}$ gas and the metal surface, the $\mathrm{H}_{2}$ is dissociated (heat of dissociation $\mathrm{E}_{\mathrm{D}}=218 \mathrm{~kJ} / \mathrm{mol} \mathrm{H}$ ) and chemisorbed at the surface and in the near surface sites. The flat minimum in $\left(\mathrm{H}_{2}+\mathrm{M}\right.$ curve $)$ curve represent the physisorbed $\mathrm{H}_{2}$ (heat of physisorption $E_{P} \approx 10 \mathrm{~kJ} / \mathrm{mol} \mathrm{H}$ ), and the deep minimum in the chemisorbed curve $\left(2 \mathrm{H}+\mathrm{M}\right.$ curve) describes chemisorbed, dissociated $\mathrm{H}$ (heat of chemisorption $E_{C} \approx 50 \mathrm{~kJ} / \mathrm{mol}$ $\mathrm{H})$. Chemisorption is a process in which the atoms or molecules of the reacting substrate are held to the surface atoms of the host by electrostatic forces of about the same strength as the chemical bonds. If the physisorbed and the chemisorbed potential energy curve intersect above the zero energy level, the chemisorption requires an activation energy $E_{A}$, which slows down the kinetics of dissociative adsorption and recombinative desorption.

The adsorbed $\mathrm{H}$ atoms have a high surface mobility. They interact with each other at sufficiently high coverage and form surface phases. Many surface properties such as $E_{C}$ and sticking probability becomes coverage dependent. Temperature-programmed thermal desorption (TPD) analysis done by Christman [44] on $\operatorname{Pd}(110)$ surfaces shows that at room temperature the surface phase is stable and has a coverage degree of one. Only above $325 \mathrm{~K}$ desorption from the surface occurs.

In further steps the chemisorbed $\mathrm{H}$ atoms penetrate the surface and are dissolves exothermically or endothermically in the bulk where a hydride phase may nucleate and grow. 
Near-surface region (subsurface) exist between the surface sites and the inner bulk sites. The fact that the distance between the first and the second metal atom planes can be adjusted more easily than between planes in the bulk, generally leads to an enhanced hydrogen content in this subsurface region. $\mathrm{Nb}$ and $\mathrm{Pd}$, for example are known to exhibit strongly enhanced nearsurface solubility for $\mathrm{H}[45-47]$.

To summarise, the hydrogen solubility is enhanced at the surface region.

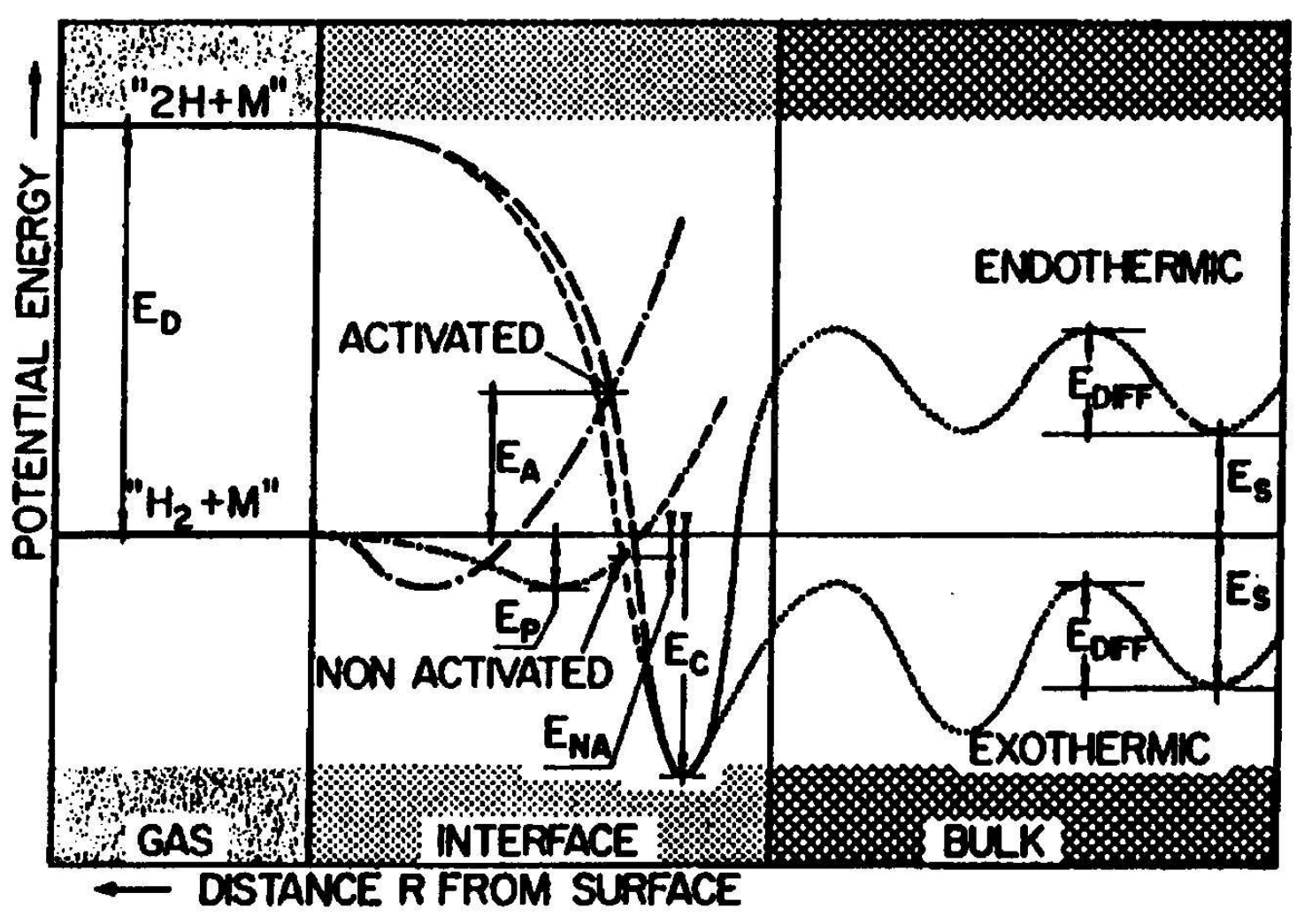

Figure 1. 5. Potential energy curves for activation and chemisorption of hydrogen on metal surface and solution in bulk after [43]. 


\subsubsection{Interaction with nanometer-sized Materials}

The notable features of hydrogen interaction with nanometer-sized materials, such as nanocrystallites, multi metallic thin layers and clusters, are: the enhanced hydrogen solubility at low concentration and the narrowing of the miscibility gap when compared to bulk system. In complex materials, such as nanocrystalline or multi metallic thin layers, disturbances of lattice by structural and chemical defects can represent interfacial traps for hydrogen. Vacancies and grain boundaries represent low energy sites (traps) [48]. The distribution of hydrogen among the different sites governed by the laws of statistical thermodynamics, where the interstitial sites are divided into different ensembles.

Nanocrystalline material was used to investigate the hydrogen palladium interaction of small grains $[4,36]$. At low $\mathrm{H}$ concentration the solubility of hydrogen is enhanced remarkably. The plateau in the $p-x-T$ isotherm occurs at the same equilibrium hydrogen pressure or chemical potential as in the ploycrystalline sample. However, the miscibility gap is narrower in nanocrystalline material. Mütschele and Kirchheim attributed this to the presence of two type of hydrogen site: normal interstitial sites in the grains and grain boundaries sites. The grain boundary sites have lower energy [6] and are occupied at lower chemical potential first. Thus the nanocrystalline sample has higher solubility. The narrowing of the miscibility gap was explained by the great number of Pd atoms residing in the grain boundaries, which do not take part in the hydride formation at higher chemical potentials.

In clusters the surface partition is very large. The percentage of surface atoms varies from $75 \%$ to about $30 \%$ in the cluster range of 1 to $4 \mathrm{~nm}$, see section (2.1.2). This surface volume, surface and subsurface atoms, will affect the sample behaviour. Pundt et al. and Sachs et al. $[7,8]$ have shown that this can be treated like the grain boundary volume. The narrowing of the miscibility gap in clusters can be explained based on the assumption that the surface and subsurface sites do not participate in the hydride formation. From the measured phase boundaries the number of subsurface atoms can be obtained. The fraction $c_{t}$ of surface sites $N_{s s}$ using the boundaries of the two-phase field is [7,8]:

$$
c_{t}=\frac{N_{s s}}{N}=1-\frac{c_{\alpha^{\prime}}^{c l}-c_{\alpha}^{c l}}{c_{\alpha^{\prime}}^{\text {poly }}-c_{\alpha}^{\text {poly }}}
$$

with $c_{\alpha^{\prime}}^{\text {poly }}=0.607 \mathrm{H} / \mathrm{Pd}$ and $c_{\alpha}^{\text {poly }}=0.008 \mathrm{H} / \mathrm{Pd}$ are the concentration at the phase boundaries of hydrogen phase diagram of polycrystalline $\mathrm{Pd} . c_{\alpha^{\prime}}^{c l}$ and $c_{\alpha}^{c l}$ are the concentrations at the phase boundaries of the Pd cluster hydrogen system. 


\section{Metal Clusters}

Clusters are aggregated atoms or molecules, generally intermediate in size between individual atoms and aggregates large enough to be called bulk matter [49]. In temptation to offer distinction between clusters and colloids, scientists defined clusters as aggregates of atoms in the range of 1-10 $\mathrm{nm}$. Whereas, colloids ${ }^{2}$ have a size range of 1-20 $\mathrm{nm}$ [50]. In 1861 Graham [51] use the term "colloid or glue-like" to describe the slow diffusion rates and noncrystallisation in aqueous solutions of insoluble materials, such as silver chloride and gold. In the following chapter the cluster structures in terms of closed shell model and the magic numbers will be viewed. The preparation methods of nanometer-sized metallic cluster and the clusters stabilisation methods will be also examined. Both physical and chemical preparation methods will be surveyed. This will be followed by discussing the mechanism and a thermodynamic approach of electrochemical phase formation.

\subsection{Cluster structures}

The structure of clusters can be interpreted in terms of the shell models. It was observed that mass spectra of alkali and noble metal clusters have anomalies of abundance in the intensity which occurs at a specific sizes $N$, called magic numbers. This indicates that nanoclusters of these sizes are relatively stable as compared with those of the neighbouring sizes [52].

The magic numbers can arises from "electronic shell structure" or "atomic shell structure".

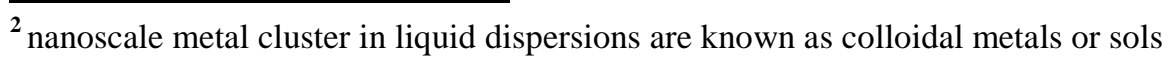




\subsubsection{Electronic shell structure}

The idea that clusters which exhibit high stability correspond to a particular "closed shell" electronic configuration is basic for the use of the jellium model [12]. This model describes the cluster electronic structure by considering only the valence electrons which are assumed to move "freely" in a smooth attractive mean field potential. The jellium model been has successfully applied to the cluster ion distributions observed in alkali and s-bonded metal clusters such as those of $\mathrm{Cu}, \mathrm{Ag}$ and $\mathrm{Au}[53]$.

\subsubsection{Atomic shell structure}

This model is based on the assumption that clusters of particular size have structures that correspond to closed shells of component particles. In the following two possible geometries the icosahedral and cubic structure will be discussed.

According to this model the number of atoms $N$ in a cluster can be calculated according to:

$$
N=1+\sum_{1}^{i}\left(10 n^{2}+2\right)
$$

where $n(n \geq 1)$ is the shell number of the cluster. The number of atoms at the surface of the cluster $N_{s}$ is equal to the number of atoms in the latest shell:

$$
N_{s}=10 n^{2}+2
$$

Assuming that the cluster is approximately spherical, the diameter of a cluster of $\mathrm{N}$ atoms is given by [54]:

$$
d(N)=2 r_{w s} \cdot N^{1 / 3}
$$

where $r_{w s}$ is the Wigner-Seitz radius of the element under consideration (i.e. the radius of a sphere whose volume is equal to the volume per atom in the solid), see Table 2.1. 
Table 2. 1. The relation between the number of atoms in a cluster of $n$ shell

with the surface atoms $N_{s}$ and the diameter $d$.

\begin{tabular}{|c|c|c|c|}
\hline$n$ & $N$ & $N_{s}$ & $\mathrm{~d}[\mathrm{~nm}]$ \\
\hline 1 & 13 & 12 & 0.70 \\
\hline 2 & 55 & 42 & 1.13 \\
\hline 3 & 147 & 92 & 1.56 \\
\hline 4 & 309 & 162 & 2.00 \\
\hline 5 & 561 & 252 & 2.44 \\
\hline 6 & 923 & 362 & 2.88 \\
\hline 7 & 1415 & 492 & 3.33 \\
\hline 8 & 2057 & 812 & 3.77 \\
\hline 9 & 2869 & 1002 & 4.21 \\
\hline 10 & 3871 & 1212 & 4.65 \\
\hline 11 & 5083 & 1442 & 5.09 \\
\hline 12 & 6525 & 1692 & 5.53 \\
\hline
\end{tabular}

In agreement with this model of shell structure mass spectroscopic measurements have shown that clusters are more stable if the number of atoms is a magic number $(13,55,147,309,561$ ..... ) $[55]$.

The smallest possible icosahedral cluster consists of 13 atoms, one at the center and 12 others on each of the 12 equivalent corners of the icosahedron. The icosahedron can be constructed from 20 slightly distorted $f c c$ unit cells, whose faces are (111) planes and which share common vertices.

Icosahedral packing arrangements have many favourable attributes [56]. The icoshedron has the highest symmetry of all discrete groups. The number of nearest neighbours in an icosahedron is larger than in an $f c c$ lattice and the surface energy is smaller [10]. Each of the 20 triangular faces in the icosahedron can be constructed with close-packed spheres. On the other hand, the icosahedron is a highly strained structure. The interatomic distances between shells is smaller than within a shell. For this reason, icosahedral packing is found only in relatively small clusters having a high surface-to-volume ratio, so that for larger clusters the cubic lattice becomes favoured. 
During cluster growth both structures can be obtained depending on the growth path. So, experimentally both structures can appear. Duff [57] proposed that nucleation based on a ploytetrahedral nucleus will lead to iscoahedral geometry. That means the nucleation process goes as far as Figure 2.1 (g) or even further before normal metallic growth commences. On the other hand, if the metallic growth takes place once the structure in Figure 2.1 (b) is reached, a normal $f c c$ is obtained.

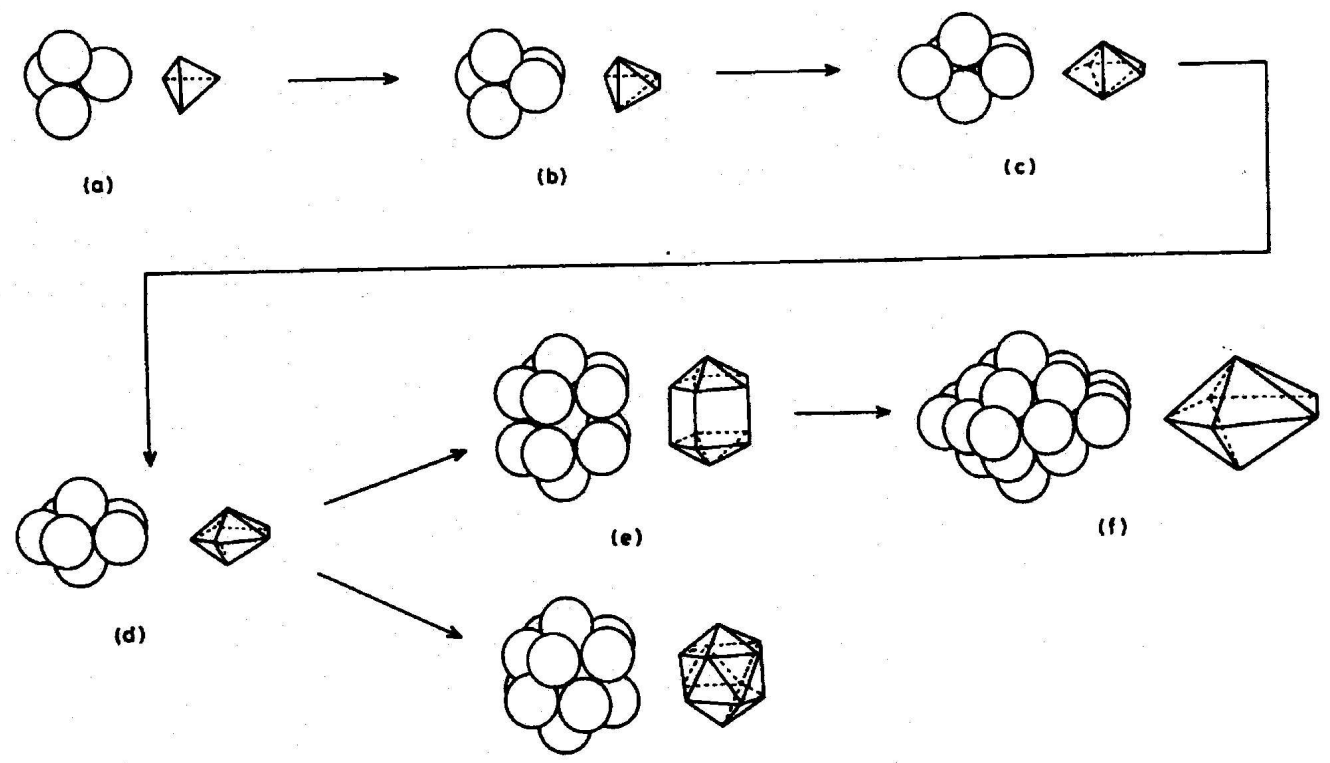

(g)

Figure 2. 1. Successive addition of single atoms to a tetrahedral cluster (a) leads to a triognal bipyramid (b), a capped trigonal bipyramid (c), and a simple decahedron or pentagonal bipyramid (d). Further addition of atoms in normal metallic packing leads to development of decahedron $(e)$ and (f) or if tetrahedral packing is maintained, to the icosahedron $(g)$. 


\subsection{Clusters stabilisation methods}

Nanoparticles tend to be fairly unstable, in solution, with respect to agglomeration to the bulk. So special precautions have to be taken to avoid their aggregation. All clusters preparation methods involve the use of stabilising agents, which are adsorbed at the surface of the particle. The charge or solubility properties keep the clusters suspended ${ }^{3}$, and thereby prevent their aggregation. Stabilisation can be achieved by two methods: electrostatic stabilisation and steric stabilisation.

\subsubsection{Electrostatic stabilisation}

Anions and cations from the starting materials remain in solution, and associate with the metal particles. The metal particles are surrounded by an electrical double layer, due to adsorbed negatively charged ions and cations which are attracted to them. This result in a Coulombic repulsion between particles that varies approximately exponentially with the interparticle distance as shown schematically in Figure 2.2. The week minimum in potential energy at moderate interparticle distance defines a stable arrangement of metal particles that is easily disrupted by medium effect and, at normal temperature, by the thermal motion of the particles. Thus if the electric potential associated with the double layer is sufficiently high, electrostatic repulsion will prevent particle agglomeration.

A dispersion of electrostatically stabilised metal clusters can be coagulated if the ionic strength of the dispersing medium is increased sufficiently, since this compresses the double layer and shortens the range of the repulsion. The stabilising effect of the surface ions dependents on their concentration, and if the surface charge is reduced by the displacement of adsorbed anions by a more strongly binding neutral adsorbate the cluster particles can now collide and agglomerate under the influence of the van der Waals forces.

Even in less polar organic media, in which electrostatic effects would not normally be considered important, a charge will develop on a metal surface in contact with organic phases

\footnotetext{
${ }^{3}$ At short interparticle distances two particles would attracted to each other by van der Waals forces and, in the absence of repulsive forces to counteract this attraction, unprotected clusters would coalesce.
} 


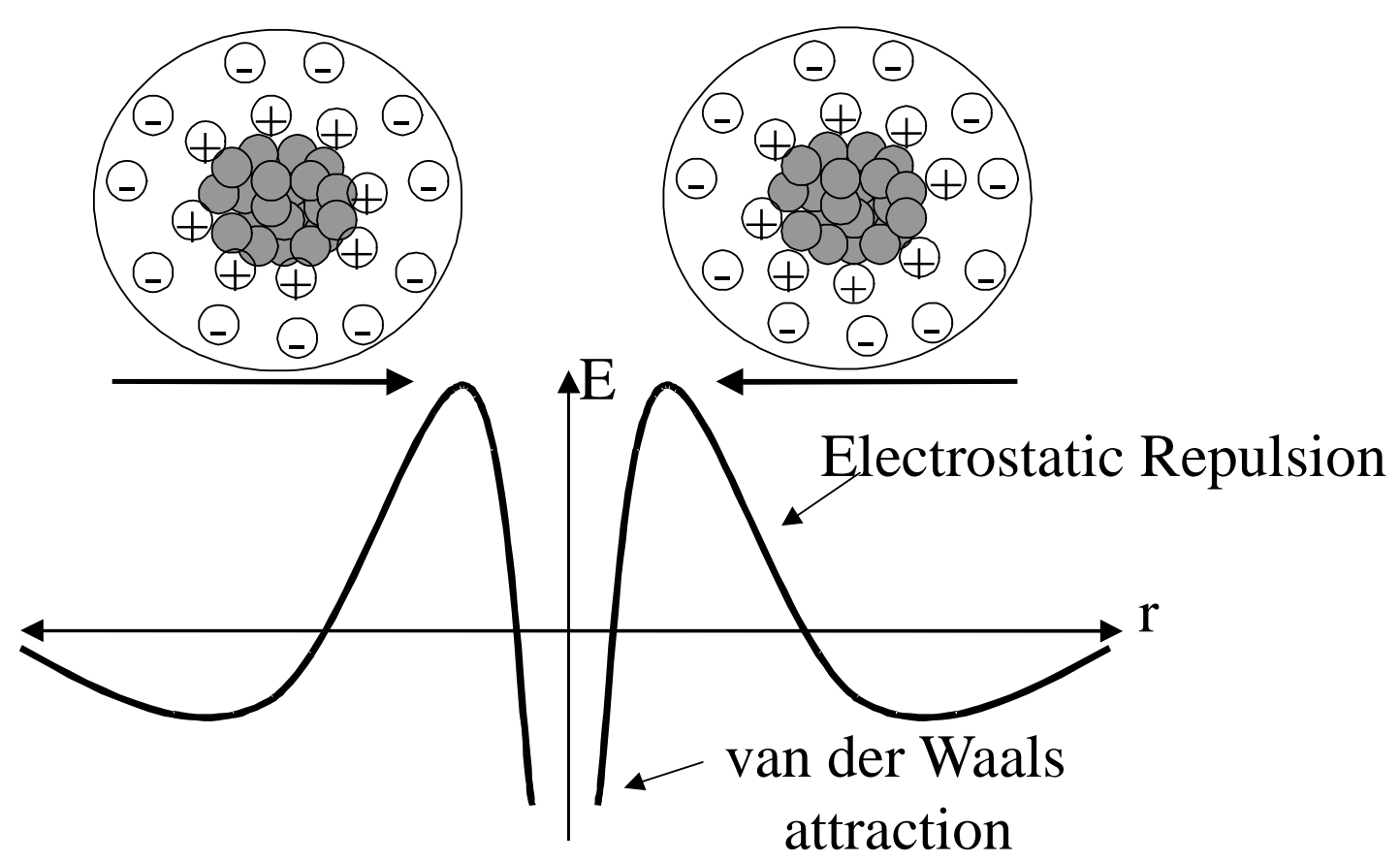

Figure 2. 2. Electrostatic stabilisation of metal nano-clusters. The attractive van der Waals forces are outweighted by the repulsive electrostatic forces between the electrical double layer.

such as solvent and polymers [58]. For example the acquisition of charge by gold particles has been measured, and the sign and magnitude of the charge is found to vary as a function of the donor properties of the liquid. Thus, even for particles in suspension in relatively non-polar liquids the possibility cannot be excluded that electrostatic stabilisation is present.

\subsubsection{Steric stabilisation}

A second means by which metal particles can be prevented from aggregating is by the adsorption of large molecules such as polymers or surfactants at the surface of the particles, thus providing a protective layer. The way in which adsorbed large molecules prevent aggregation can be seen in a simplified manner by visualising the close approach of two particles, each with long chain molecules adsorbed at their surfaces as shown in Figure 2.3. In the inter-particle space the adsorbed molecules would be restricted in motion, fewer conformation will be accessible, causing a decrease in entropy and thus an increase in free energy. A second effect is caused by the local increase in concentration of adsorbed molecules as the protective sheaths on each particle begin to interpenetrate. This causes an osmotic repulsion as the solvent reestablishes equilibrium diluting the stabiliser molecules, separating 
the particles. It can be seen that the stabiliser molecule in order to function effectively, must not only coordinate to the particle surface, but also be adequately solvated by the dispersing fluid.

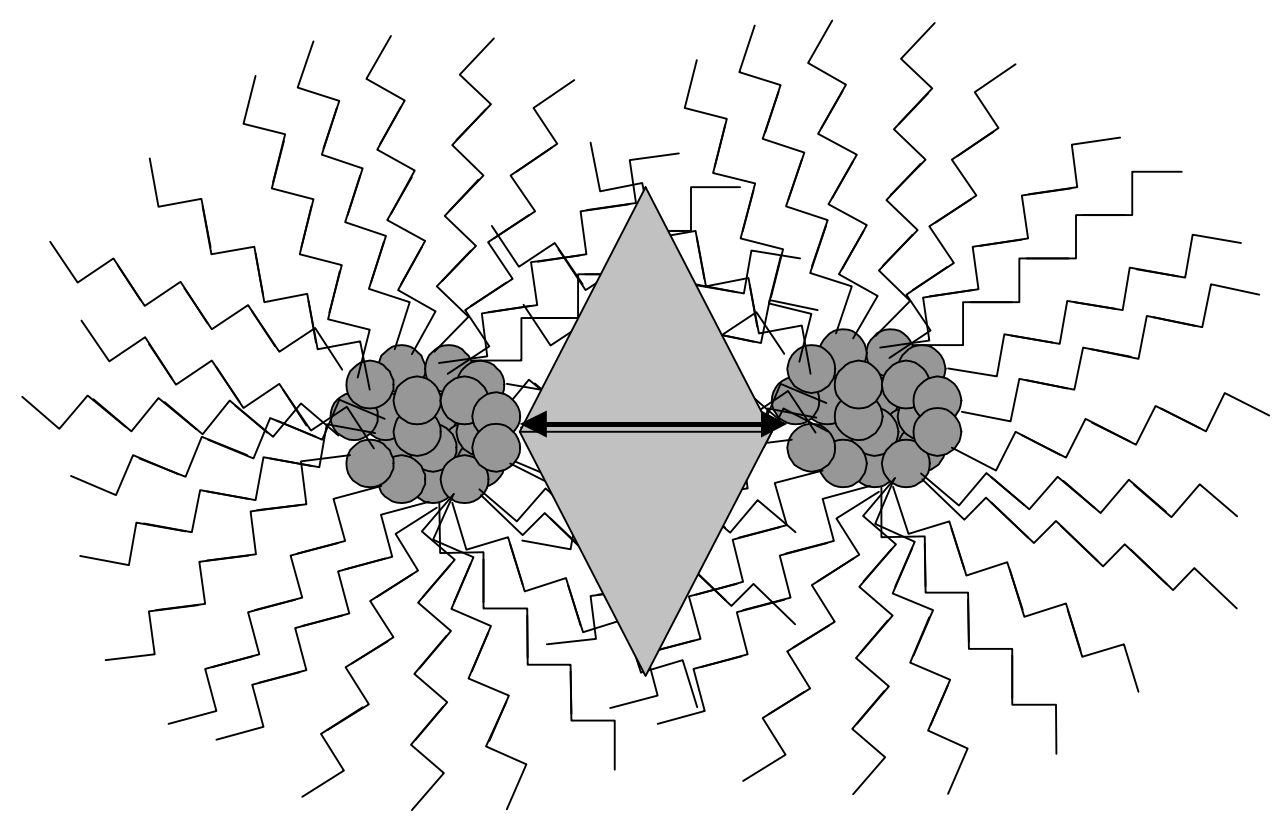

Figure 2. 3. Steric stabilisation of metal nano-clusters. The adsorbed large molecule on the nanoparticles surface provide a protective layer and prevent them from aggregation.

In this work we are using tetraoctylammonium bromide $\mathrm{Oc}_{4} \mathrm{~N}^{+} \mathrm{Br}^{-}$as a stabiliser. Thus we expect that the Pd clusters are mainly stabilised by steric stabilisation. The Pd clusters are prepared in polar solvent acetonitrle or tetrahydrofuran or a mixture of both. Thus, a charge can develop on the metal nanoparticle surface and therefore electrostatic stabilisation can not be excluded. 


\subsection{Clusters preparation methods}

In principle the clusters preparation methods could be classified in two main methods: physical methods and chemical methods.

\subsubsection{Physical methods}

The most popular physical methods are those using metal-vapour-co-condensation technique, where zero-valent metal atoms are allowed to interact and form clusters in a liquid dispersion. Clusters can be made by a vapour of the elementary component particles which are allowed to aggregate, or by knocking them directly out of a solid. However most of the work since late 1960's has dealt with gaseous clusters. The beam source of free clusters can be: i) supersonic jet, ii) gas aggregation source, a less common source is iii) sputtering.

\subsubsection{Chemical methods}

The standard method for colloid formation are based on the chemical reduction of transition metal salts using such different reducing agents as hydrogen, alcohol [59], hydrazine or borohydrides [60,61] in the presence of stabilisers such as special ligands [62], polymers [63] or surfactants [64] of the type tetraalkylammonium halide $\left(\mathrm{R}_{4} \mathrm{~N}^{+} \mathrm{X}^{-}\right)$which prevent undesired agglomeration and formation of metal powders. Generally, the actual size of the nanoparticles obtained varies from system to system. An observation which is not easy to explain, especially in view of the fact that not only the reducing agent and the nature of the metal varied but also other parameters such as solvent, concentration, temperature and reaction time. 


\subsubsection{Reduction of metal salts}

$$
x M^{n+}+n x e^{-} \stackrel{\text { stabiliser }}{\longrightarrow} M_{n(\text { cluster })}^{0}
$$

The chemical reduction of transition metal salts in the presence of stabilising agents to generate zero-valent metal colloids in aqueous or organic media was first published in 1857 by Faraday [65] and this approach has become one of the most common and powerful synthetic methods in this field [50,66,67]. Turkevich and co-workers [61] were the first to establish reliable procedures for the preparation of metal colloid ( e.g. $20 \mathrm{~nm}$ gold by reduction of $\left[\mathrm{AuCl}_{4}\right]^{-}$with sodium citrate). They also proposed a mechanism for the stepwise formation of nano-clusters based on nucleation, growth and agglomeration, which in essence is still valid.

\subsubsection{Thermal or photochemical decomposition of organometallic precursors}

$$
\begin{gathered}
n M^{0}(L) x \stackrel{\text { stabiliser }}{\longrightarrow} M_{n_{(\text {cluster })}}^{0}+x L \\
n F e(C O)_{5} \stackrel{\text { polymer }}{\longrightarrow} F_{n(\text { cluster })}^{0}+5 n C O
\end{gathered}
$$

A wide variety of organometallic complexes can be decomposed thermally as in the above equations [68]. Photolysis is used much less frequently as a cluster preparatory method.

\subsubsection{Reduction of organic ligands in organometallic precursors}

$$
\begin{gathered}
n M^{0}(L) x+x H_{2} \stackrel{\text { stabiliser }}{\longrightarrow} M_{n(\text { cluster })}^{0}+x L H_{2} \\
n N i\left(C_{8} H_{10}\right)_{2}+2 H_{2} \stackrel{\text { polymer }}{\longrightarrow} N i_{n(\text { cluster })}^{0}+2 n C_{8} H_{14}
\end{gathered}
$$

In this method the ligands are reduced in a way that they will no longer bind to the zerovalent metal atom. Then the free metal atom can combine with other metal atoms to form metal nano-clusters [69]. 


\subsubsection{Metal vapour chemistry}

In this method the atomic vapour of a metal is condensed into a cold liquid which contains a stabiliser. In the case that the liquid itself functions as a stabiliser the metal vapour can cocondense with the solvent vapour to give a solid frozen matrix. On warming the dissolved metal atoms aggregate to form metal clusters [70].

\subsubsection{Electrochemical method}

An electrochemical method for the preparation of tetraalkyammonium halide $\left(\mathrm{R}_{4} \mathrm{~N}^{+} \mathrm{X}^{-}\right)$ stabilised transition metal clusters has been developed [64] which allows the control of the particle size simply by selection of the current density. This technique has many advantages as: high yield, absence of side products, the cluster can easily be isolated and different cluster sizes can be prepared in selective manner simply by the variation of the current density. Furthermore, the clusters are stabilised in elastically soft stabiliser.

Because of the given advantages the electrochemical technique will be used in this work to produce Pd clusters of different size. Details are given in following sections.

\subsection{Mechanism of cluster formation}

Many electrode reactions involve the formation of a solid phase, either as the result of the reduction of ions, as in the case of metal deposition or electrochemical cluster preparations, or by oxidation of the electrode and subsequent anion to form an anodic film [73].

The electrochemical preparation of Pd clusters, as well as other electrode reactions, involves the following distinct steps:

1) Oxidative dissolution of the Pd anode.

2) Migration of the $\mathrm{Pd}^{2+}$ ions to the cathode.

3) Electron transfer and partial or complete loss of the solvation sheath, resulting in the formation of ad-atoms.

4) Formation of metal nano-particle by nucleation and growth. 
5) Arrest of growth process and stabilisation of the nano-particles by cluster protecting agents $\mathrm{Oc}_{4} \mathrm{~N}^{+} \mathrm{Br}^{-}$.

6) Precipitation of the clusters.

These steps can be schematically represented as shown in Figure 2.4.

The overall process can be summarised in the following two steps of an oxidation-reduction reaction:

$$
\begin{aligned}
& P d_{\text {Anode }} \rightarrow P d^{2+}+2 e^{-} \\
& P d^{2+}+2 e^{-}+\text {stabiliser } \rightarrow P d_{\text {cluster/stabiliser }} \\
& -------------------- \\
& P d_{\text {Anode }}+\text { stabiliser } \rightarrow P d_{\text {cluster/stabiliser }}
\end{aligned}
$$

Electrode processes have been employed in the preparation of insoluble metal powders, usually in aqueous acidic medium [71]. In contrast, in this work we use a Pd anode as the metal source in a simple electrolysis cell, where $\mathrm{Oc}_{4} \mathrm{~N}^{+} \mathrm{Br}^{-}$serving as the electrolyte and as the stabiliser in an organic solvent. The $\mathrm{Oc}_{4} \mathrm{~N}^{+} \mathrm{Br}^{-}$prevents the reduced $\mathrm{Pd}$ nano-particles from aggregation into bulk. This results in stabilised Pd clusters rather than an insoluble metal powders.

Pd-Anode

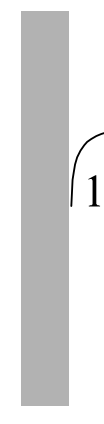

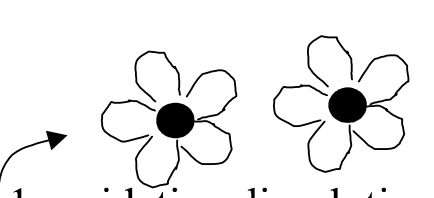

1 oxidative dissolution
2. migration
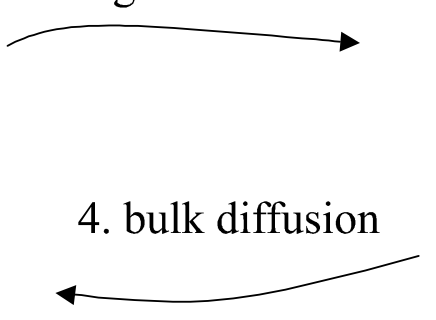

4. nucleation and growth
Cathode

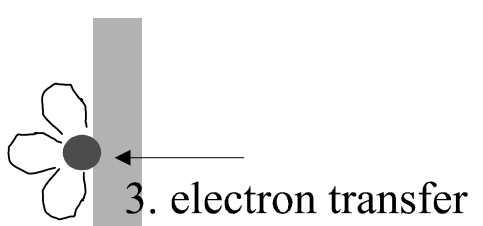

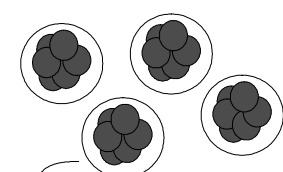

5. arrest of growth and stabilisation

6. precipitation

Figure 2. 4. Schematic representation of the electrochemical preparation of $\mathrm{Oc}_{4} \mathrm{~N}^{+} \mathrm{Br}-$-stabilised $\mathrm{Pd}$ cluster. 


\subsection{The Thermodynamics of Electrochemical Phase}

\section{Formation}

It is well known that the size of a colloidal particle obtained by metal vapour method depends on the metal evaporation rate [72]. This can be described using the Kelvin equation. Where the particles size is affected by the saturation of the gas phase with the metal atoms. The higher the saturation, the smaller the expected particles.

$$
\ln \left(\frac{p_{r}^{*}}{p_{\infty}^{*}}\right)=\left(\frac{2 \gamma \bar{V}}{R T}\right) \cdot \frac{1}{r}
$$

where:

$\gamma=$ the molar surface free energy (surface tension)

$p_{r=\text { the equilibrium vapour pressure at experimental conditions }}^{*}$

$p_{\infty}^{*}=$ the equilibrium vapour pressure as $1 / \mathrm{r} \rightarrow 0$

$V=$ is the molar volume of the condensed phase

$r=$ is the particles size

In the case of electrochemical phase formation, equilibrium is established due to electron transfer ${ }^{4}$. If the electrode process of the type, $M^{n+}+n e^{-} \Leftrightarrow M$

occurs, the equilibrium electrode potential $E_{e q}$ is given by the Nernst equation:

Under these equilibrium conditions, the surface activity of ad-atoms has it is equilibrium value $a_{M_{a d s}}^{*}$.

\footnotetext{
${ }^{4}$ Since the growth of the new phase occurs at the boundary between the electrode material and the solution, the nucleation process must be heterogeneous. Although it is necessary to take the properties of the substrate into account, it is also instructive to pursue the analogy with homogeneous nucleation from the vapour by assuming that the contact area between nuclei and the electrode is vanishingly small; i.e that small spherical droplet are formed on a foreign electrode material.
} 
If the electrode potential is changed to some new value $\left(E_{e q}+\eta\right)$, where $\eta$ is the overpotential, the surface activity of ad-atoms will be determined by the relation [73]:

$$
\frac{a_{M_{\text {ads }}}}{a_{M_{\text {ads }}}^{*}}=\exp \left(-\frac{n F \eta}{R T}\right)
$$

where, $n$ is the valency, $F$ is the Faraday constant $\left(9.6485309 \times 10^{4} \mathrm{C} / \mathrm{mol}\right), \quad \eta$ is the overpotential., $R$ is the gas constant $(8.314510 \mathrm{~J} / \mathrm{mol} \mathrm{K})$ and $T$ is the temperature.

The specific free energy of formation of the condensed phase is:

$$
\Delta g_{v}=\frac{n F \eta}{\bar{V}}
$$

sitting $\quad \bar{V}=\frac{M}{\rho}$ where $M$ is the molecular weight and $\rho$ is the density of the deposit:

$$
\Delta g_{v}=\frac{n F \rho \eta}{M}
$$

The growth of a metal cluster is determined by two factors. On the one hand the growth of a metal particle is favoured by the increase of the molar volume energy $\Delta G_{b u l k}$, and on the other hand it is not favoured by the surface energy. The free energy of a cluster is thus determined by it molar volume energy and its opposite surface energy. The surface energy $\Delta G_{\text {surf }}$ can be defined simply as a product of the surface tension (the specific surface free energy) $\gamma$ and the surface area of a sphere

$$
\Delta G_{\text {surf }}=4 \pi r^{2} \gamma
$$


The molar volume energy increases with increasing cluster volume as follows:

$$
\Delta G_{b u l k}=\Delta g_{v} \cdot \frac{4}{3} \pi r^{3}=\frac{4 \pi \rho \eta n F}{3 M} r^{3}
$$

where $\rho$ is the density and $M$ is the mass. The overpotential $\eta$ is defined as the deviation from the standard potential: $\eta=E^{*}-E$. The free enthalpy of a metal cluster $\Delta G_{\text {cluster }}$ can therefore described as:

$$
\Delta G_{\text {cluster }}=\frac{4 \pi \rho \eta n F}{3 M} r^{3}+4 \pi r^{2} \gamma
$$

It becomes clear that with radii $(r<<1)$ the surface term outweighs. To grow very small particles is thus difficult. By contrast, with larger radii, the fast increasing volume enthalpy determines growth.

$\Delta G_{\text {cluster }}$ of a growing cluster goes through a maximum, Figure 2.5. This point is called the critical radius $r_{\text {crit }}$. The critical radius can be obtained by equating the derivative of equation (2.10) to zero.

$$
r_{c r i t}=\frac{2 M \gamma}{n F \rho \eta}
$$

Particles with a radius smaller than $r_{c r i t}$ are unstable. To grow these particles energy is needed, because they must overcome the surface energy necessary for the enlargement of the surface. Particles can be prevented within this size range from growing further by using a stabilisers. In contrast to this, particles which already crossed the critical radius grow very fast. 
With high overpotentials, the critical radius decreases and small cluster should be stabilised. Low overpotentials, however, will increase the critical radius, and particles with a larger radius should be obtained. By varying the current density, i.e the overpotential, and using a stabiliser it is possible to perform size-selective synthesis of nanometer sized clusters.

Helbig [74] succeeded by using this concept to synthesis palladium and nickel cluster in the size range of 1.3-4.5 $\mathrm{nm}$.

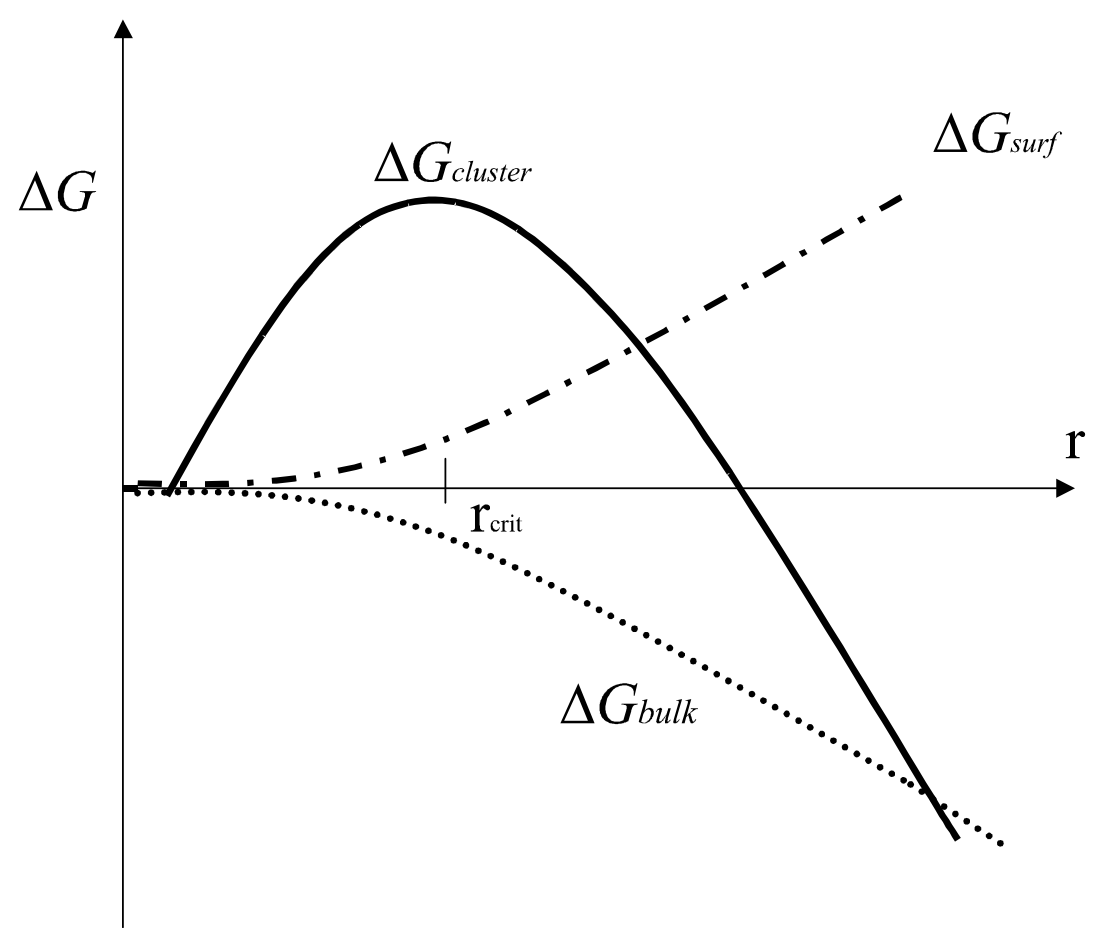

Figure 2. 5. Free energy curves of a nano-cluster as a function of its radius where the contribution to $\Delta G_{\text {cluster }}$ made by the $\Delta G_{\text {surf }}$ and $\Delta G_{\text {bulk }}$ are shown on a reduced scale by a factor of ten [73]. 


\section{Experimental Techniques and Methodology}

In this chapter the applied experimental techniques will be shown. First, a general procedures for the electrochemical cluster preparation will be presented. This will be followed by describing the set-ups used in this study. The experimental characterisation techniques used in this study will be also presented.

\subsection{Chemicals and materials}

All chemicals were purchased in it is dry pure form, or dried using the standard drying procedure. Acetonnitril $\left(\mathrm{CH}_{3} \mathrm{CN}\right)$ and terhydrofuran (THF) were purchased from Aldrich company in it is pure dry form. Tetraoctylammonium bromide $\left(\mathrm{Oc}_{4} \mathrm{~N}^{+} \mathrm{Br}^{-}\right)$was purchased from Fulka company and dried prior to use. Palladium (Pd) was purchased from Goodfellow company.

\subsection{Electrochemical cluster preparation}

The electrochemical preparation process was done in a two-electrode setup (electrolysis cell) consisting of two geometrically equal Pd electrodes Figure 3.1 . The cell is a $150 \mathrm{ml}$ standard electrolysis cell (\#6.1415.250) purchased from Metrohum, which was then redesigned in our mechanic work shop. The electrolysis cell has a four-outlet Teflon cover that closes it tightly. Two to make the electrical contact with the electrodes, one to charge (or discharge) the cell with the reaction mixture, and one for the argon gas inlet. 


\subsubsection{General procedures}

In a typical experiment, the electrolytic cell was charged with $90 \mathrm{ml}$ of $0.1 \mathrm{M}$ tetraoctylammonium bromide in $\mathrm{CH}_{3} \mathrm{CN}$ or THF. All operations were performed under inert gas atmosphere (argon). Two sheets of pure palladium $\left(2.5 \times 2.7 \mathrm{~cm}^{2}\right.$ geometric electrode surface area and $0.25 \mathrm{~mm}$ thickness) at a specific distance were used as the electrodes. A constant current was then passed between the palladium electrodes. By means of a thermostatic water bath, the electrolytic cell is maintained at the desired temperature. After running the reaction for a certain period of time, the electrolysis is stopped and the electrolyte is pressed to a $250 \mathrm{ml}$ nitrogen flask, Fig. 3.2(C). The electrolyte is allowed to set for 24 hours. Afterwards the clear supernatant is siphoned off, Fig.3.2 (D). The precipitate is then dried under high vacuum for about 3 hours.

Figure 3.1 shows a schematic representation of the tank in which the electrolysis is carried out.

All the electrolysis steps mentioned above were carried out either under argon atmosphere or vacuum for this purpose an argon-vacuum system was constructed, see Figure 3.2.

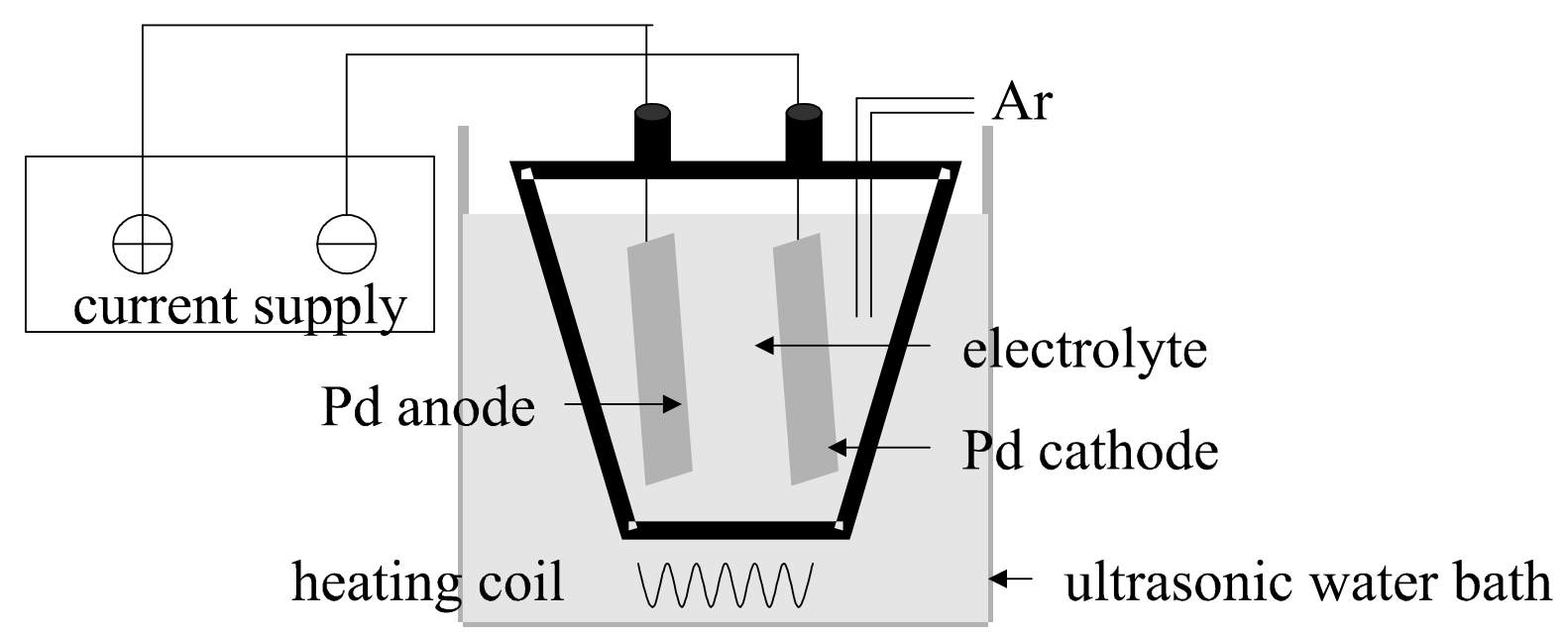

Figure 3. 1. Schematic representation of electrochemical electrolysis cell used in palladium clusters preparation. 


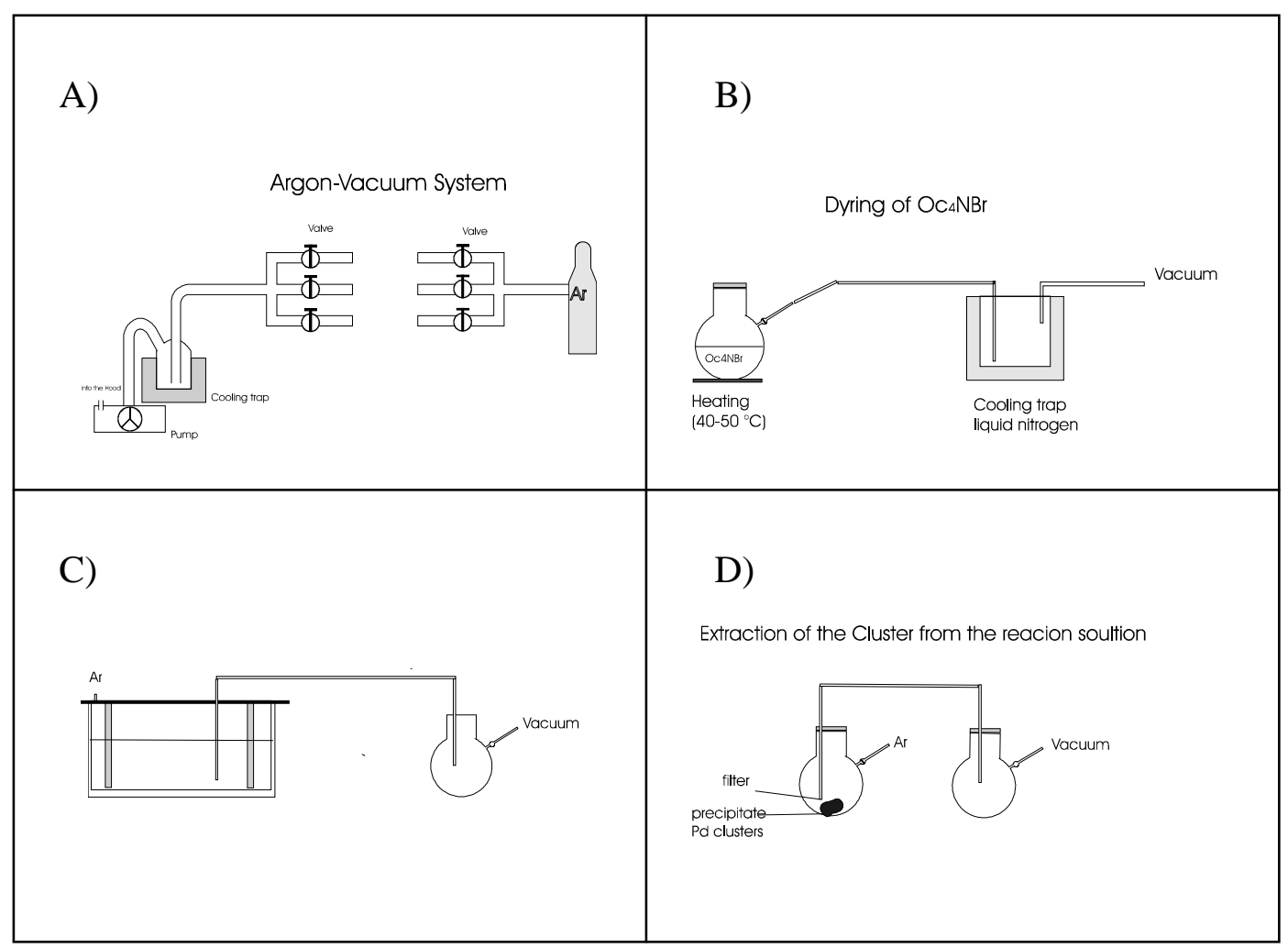

Figure 3. 2. Schematic draws of: the gas-vacuum handling system (A). drying procedure of $\mathrm{Oc}_{4} \mathrm{~N}^{+} \mathrm{Br}$ $(B)$,pressing the electrolyte into nitrogen flask and siphoning the electrolyte $(C)$, extraction of clusters from the reaction solution $(D)$.

\subsection{Experimental characterisation techniques}

\subsubsection{Transmission-electron-microscopy (TEM)}

TEM was performed with a Philips EM 420 ST microscope (resolution limit $0.3 \mathrm{~nm}$, information limit $0.2 \mathrm{~nm}$, accelerating voltage $120 \mathrm{kV}$ ). Samples were prepared by scooping them from the solution on a TEM grid $(\phi=3 \mathrm{~mm}, 400 \mathrm{mesh})$ that is coated with carbon film. The method of preparation included dipping the grid in a solution of a tetraoctylammonium bromide stabilised palladium clusters (about $10^{-4} \mathrm{M}$ ) for a few seconds. This was followed by the removal from the solution. Afterwards, the grid was dried at room temperature for several hours.

HREM was then performed using Philips CM200 FEG (FEG= field emission gun) with an accelerating voltage of $200 \mathrm{kV}$. 


\subsubsection{X-ray diffraction measurements (XRD)}

Using X-ray diffraction, the lattice structure of a solid can be investigated. In this work this method was also used to determine the cluster size.

$\mathrm{X}$-ray diffraction was performed at beam line B2 at the Hamburger synchrotron laboratory HASYLAB at DESY in Hamburg. The wavelength was selected by a Ge(111) double-crystal monochromator.

All the measurements were done in the $\theta / 2 \theta$ geometry. In this geometry, the $\theta / 2 \theta$ angles were scanned as one measured the transmitted X-ray intensity.

The geometry of the sample was adjusted that only the measured scatted intensity is from the lattice plane $\{$ hkl $\}$, which fulfils the Bragg condition $2 d_{h k l} \sin \theta=n \lambda \quad$ (where $\lambda$ is the wavelength of $\mathrm{X}$-ray beam and $d_{h k l}$ is the lattice constant). For cubic structure the lattice parameter $a$ is then:

$$
d_{h k l}=\frac{a}{\sqrt{h^{2}+k^{2}+l^{2}}}
$$

From the full width at half the maximum (FWHM) of the appropriate reflection, the cluster size (d) can be obtained using the Scherrer formula:

$$
d=\frac{0.9 \lambda}{B \cos \theta_{B}}
$$

where d: the cluster size, $B$ : the FWHM in radial, $\theta_{B}$ : is the position of the intensity maximum, and $\lambda$ : is the wavelength of the $\mathrm{X}$-ray beam.

\subsubsection{Elemental analysis}

The amount of Pd in the cluster sample was determined by Atomic absorption spectroscopy AAS. The measurements were performed with Perkin-Elmer, Flame AAS 5000 (Pd Hallocathode lamp, wavelength $244.8 \mathrm{~nm}$ ) at the elemental analysis department of PhilippsUniversity-Marburg. 


\subsubsection{In-situ XRD during hydrogen loading unloading}

In-situ X-ray diffraction measurements were performed at beam line B2 at the Hamburg synchrotron laboratory HASYLAB at DESY. A special high vacuum gas-loading cell was used that allows stepwise and controlled hydrogen loading as well as unloading between $10^{2}$ and $10^{5} \mathrm{~Pa}$, see Figure 3.3. Each loading cycle starts at a base pressure of about $10^{-3} \mathrm{~Pa}$, the hydrogen pressure was increased stepwise to $10^{5} \mathrm{~Pa}$, the unloading cycle was done by decreasing the hydrogen pressure stepwise to ca. $10^{-3} \mathrm{~Pa}$, Figure 3.4. All samples were pretreated with hydrogen to remove the oxide layer. The hydrogen pressure was measured using MKS pressure gauges with $0.01 \%$ precision. The purity of hydrogen gas was $99.9999 \%$, all measurements were performed at room temperature.

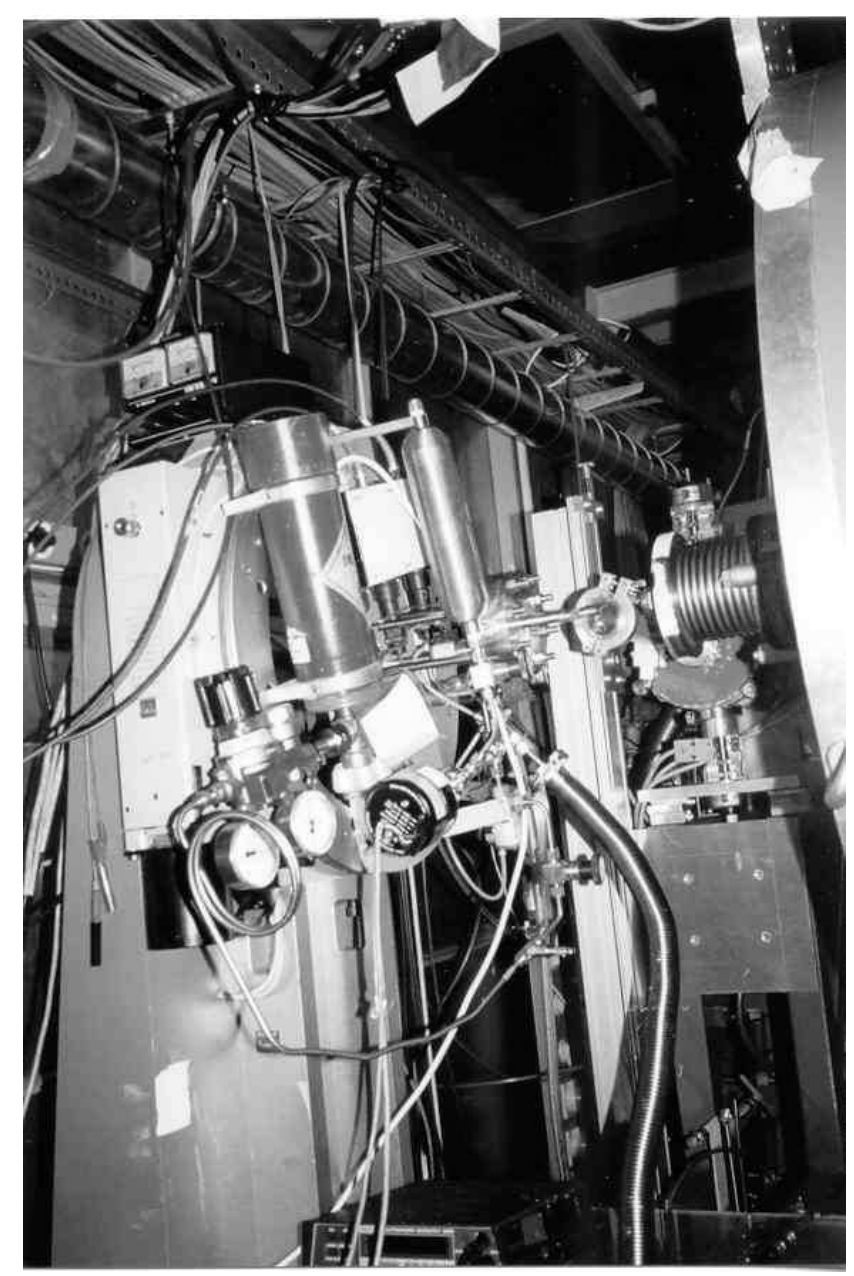

Figure 3. 3. Gas-loading cell used in the in-situ XRD measurements during hydrogen loadingunloading. 


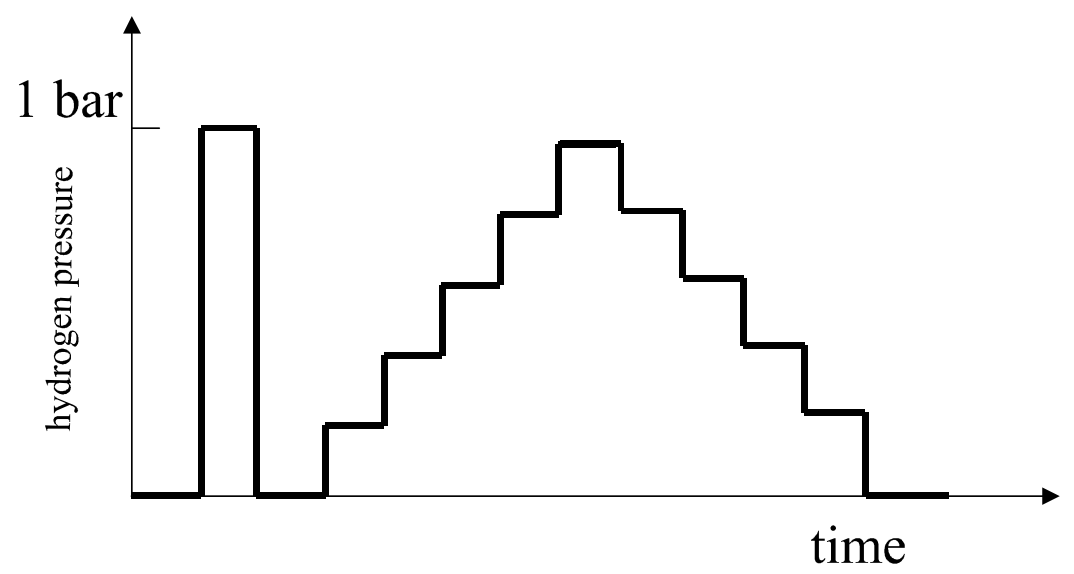

Figure 3. 4. Schematic representation of the loading-unloading cycles showing the pretreatment step.

\subsubsection{Gravimetric measurements}

The gravimetric measurements were performed using a high vacuum electronic-microbalance M-25-DV from the Sartorius company, Göttingen. The microbalance consists of two parts: measuring part (where the sample to be hanged) and a counter part, see Figure 3.5.

In a typical experiment the measuring part was charged with ca. $0.5 \mathrm{mg}$ (about $2.3 \cdot 10^{-3} \mathrm{~mole}$ palladium) palladium cluster sample were stacked into a tesa-film $\left(2.5 \times 15 \mathrm{~cm}^{2}\right)$, the counter part was also charged with an equal weight of non-adsorbing material (non-magnetic steel). The tesa-film as well as the steel solve hydrogen in a negligible amount and so do not influence the equilibrium results of the garvimetric measurements. This was checked by the weight increase of steel and tesa-film.

To reduce the oxide surface layer the cluster sample was loaded at $10^{5} \mathrm{~Pa}$ for 24 hours and subsequent evacuation for about 36 hours. This procedure was repeated at least twice.

All measurements were performed by using high purity hydrogen gas $(99.9999 \%)$ at constant temperature $\left(22 \pm 0.5^{\circ} \mathrm{C}\right)$ inside a thermostatic-controlled room. Precision gauge from MKS with the range between $10^{1}-10^{5} \mathrm{~Pa}$ was used. The sensitivity is within $0.01 \%$ of the maximum value.

The adsorption-desorption cycles were performed by loading-unloading the Pd cluster sample with hydrogen in stepwise manner, Figure 3.6. Each loading-unloading cycle consist of ca. 20 steps, where in each step the sample is loaded-unloaded with known amount of hydrogen, the weight increase and the pressure changes are monitored by time with the aid of computer program. 
Absorption of hydrogen by the sample result in an increase in the weight, which after a time interval reaches a constant value (equilibrium: constant weight at constant hydrogen pressure). The weight change is equivalent to the change in the hydrogen concentration in the sample.

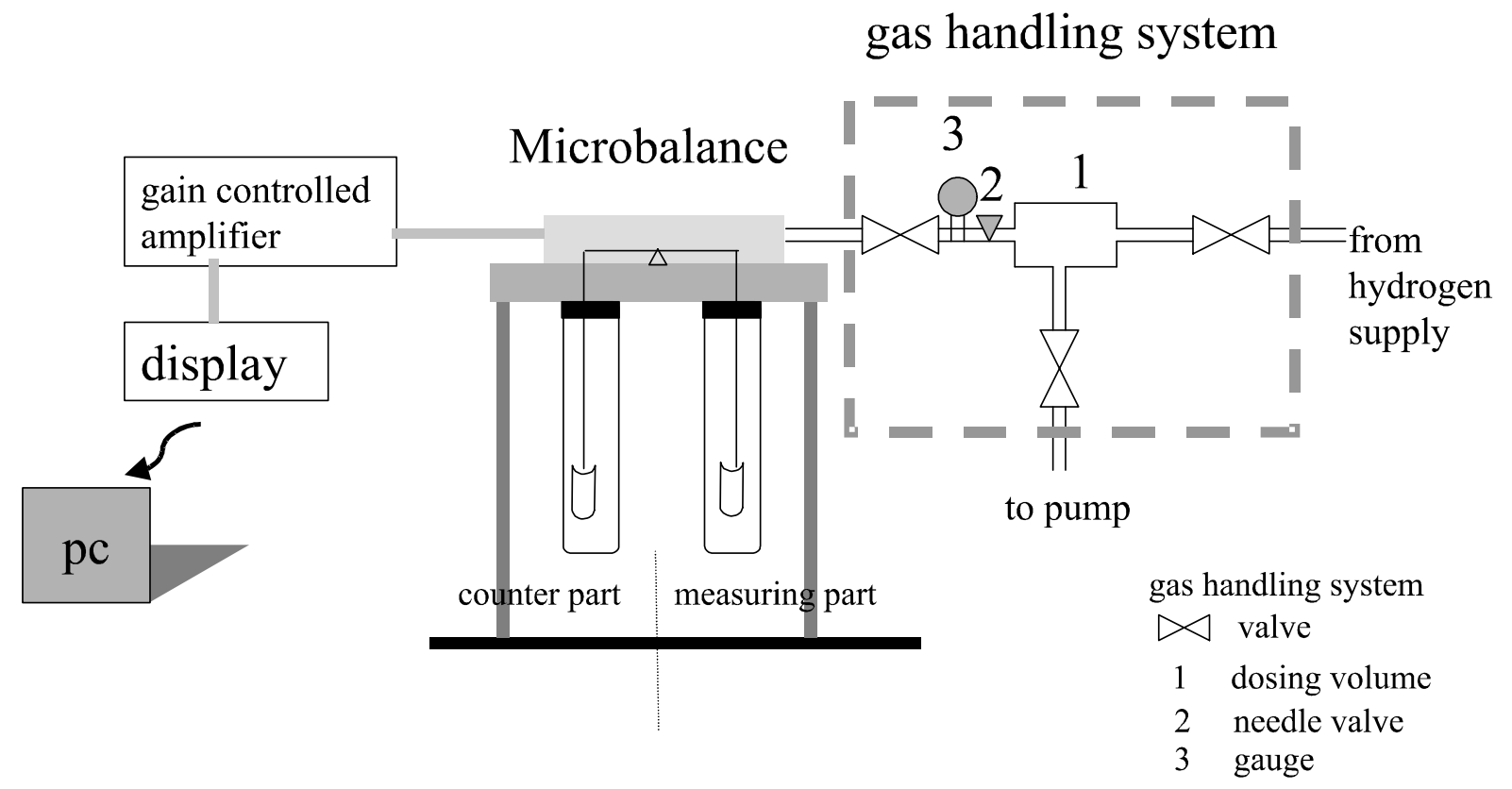

Figure 3. 5. Schematic drawing of microbalance used in the garvimetric measurements.

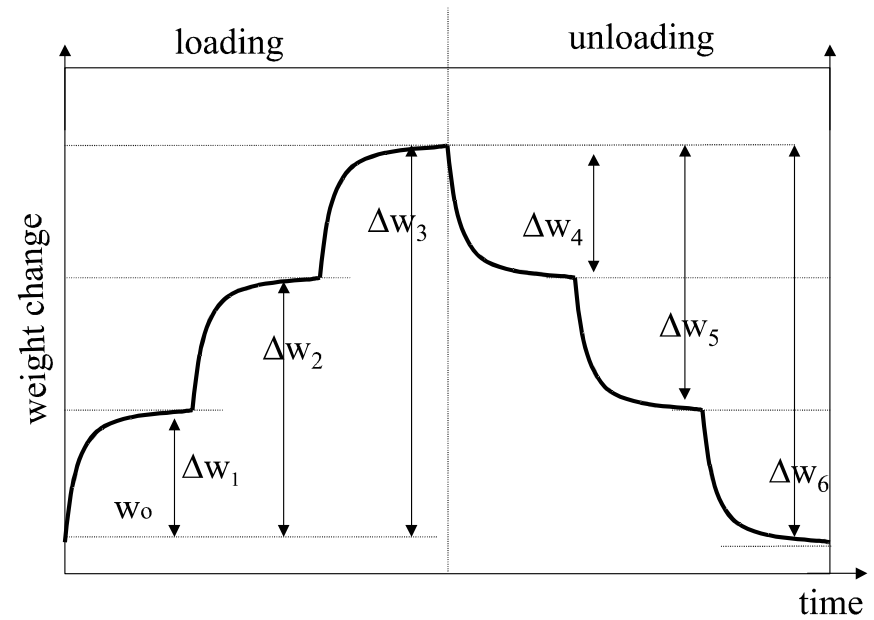

Figure 3. 6. Schematic drawing of gravimetric loading-unloading cycle. 


\section{Results: Pd Clusters Synthesis and}

\section{Characterisation}

In this chapter the results of Pd cluster preparation will be discussed. Optimisation of the electrolysis preparation parameters will be presented where the effect of different electrolysis parameters on the size as well as on the size distribution of the cluster will be studied. The cluster size, d, will be estimated by two independent methods: TEM and X-ray data analysis. The size distribution of the clusters will be determined from TEM analysis. The TEM analysis was performed with the Depp program, where more than 120 particles were considered for each cluster size. Estimation of the cluster size from X-ray diffraction data was performed utilising the full width at half the maximum (FWHM) of the main diffraction peak ((111) peak) which is inversely proportional to the size of the cluster according to the Scherrer formula.

\subsection{Electrolysis Parameters}

In this section the effect of different electrolysis parameters on the size and on the size distribution of the Pd cluster will be shown.

\subsubsection{The effect of current density}

To determine the effect of the current density $\left(I_{d}\right)$ on the cluster size $(d)$, the electrochemical preparations of Pd clusters were performed at different $I_{d}$ in $(90 \mathrm{ml}) 0.1 \mathrm{M} \mathrm{Oc}_{4} \mathrm{~N}^{+} \mathrm{Br}^{-}$in $\mathrm{CH}_{3} \mathrm{CN}$, the distance between the electrode $\mathrm{D}_{\mathrm{E}}$ was fixed to $3 \mathrm{~mm}$ and all runs were performed at $22^{\circ} \mathrm{C}$. Each run was done at a constant current $(50 \mathrm{~mA}, 25 \mathrm{~mA}, 13 \mathrm{~mA}, 5 \mathrm{~mA}$ 
and $1 \mathrm{~mA})$ corresponding to a current density ${ }^{5}:\left(5.71 \mathrm{~mA} / \mathrm{cm}^{2}, 2.86 \mathrm{~mA} / \mathrm{cm}^{2}, 1.49 \mathrm{~mA} / \mathrm{cm}^{2}\right.$, $0.57 \mathrm{~mA} / \mathrm{cm}^{2}$ and $0.11 \mathrm{~mA} / \mathrm{cm}^{2}$ ). The time allowed for electrolysis was also fixed to a charge flow (Q) of $720 \mathrm{~A} \cdot \mathrm{s}$ (except in the electrolysis at $I_{d}=0.11 \mathrm{~mA} / \mathrm{cm}^{2}$ the electrolysis was stopped at $\mathrm{Q}=116 \mathrm{~A} \cdot \mathrm{s})$.

Figure 4.1 (a-d), shows the diffraction patterns of as-prepared clusters at $I_{d}=2.86 \mathrm{~mA} / \mathrm{cm}^{2}$, $1.49 \mathrm{~mA} / \mathrm{cm}^{2}, 0.57 \mathrm{~mA} / \mathrm{cm}^{2}$ and $0.11 \mathrm{~mA} / \mathrm{cm}^{2}$, respectively. The cluster size was estimation by using the Scherrer formula, eqn. (3.2). These results show that cluster sizes in the range of 8.0 to $2.8 \mathrm{~nm}$ can be selectively prepared depending on the current density. The higher the current density the smaller the obtained cluster size see Table 4.1.

The cluster size and the size distribution was also studied by TEM, where more than 120 particles were considered for each cluster size. These results are shown in Figure 4.2 (a-e), corresponding to $I_{d}$ of: ((a) $5.71 \mathrm{~mA} / \mathrm{cm}^{2}$, (b) $2.86 \mathrm{~mA} / \mathrm{cm}^{2}$, (c) $1.49 \mathrm{~mA} / \mathrm{cm}^{2}$, (d) $0.57 \mathrm{~mA} / \mathrm{cm}^{2}$ and (e) $0.11 \mathrm{~mA} / \mathrm{cm}^{2}$. A remarkable result is that not only the cluster size is affected by the $I_{d}$, but also the size distribution. In Figure 4.2 (a) through (e), we see that the cluster size increases with decreasing the $I_{d}$. Moreover the size distribution become wider with decreasing $I_{d}$.

Table 4.1 summarises these results. It is remarkable to mention that different values are obtained from XRD and TEM for the same cluster sample. This difference in the cluster size obtained by different size estimation methods will by discussed in section (5.3).

In most of these electrolysis runs a precipitate was observed directly after the reaction was stopped. The cluster was recovered after the electrolyte to stand for 24 hour and dried under high vacuum. Only electrolysis at $5.17 \mathrm{~mA} / \mathrm{cm}^{2}$ do not form a precipitate although the $\mathrm{Pd}$ anode shows a loss of weight (308mg) after the reaction. In this case cluster size was determined from TEM where the sample was prepared from the electrolyte.

\footnotetext{
${ }^{5}$ The current density $I_{d}$ is equal to the applied current divided by the electrode area
} 

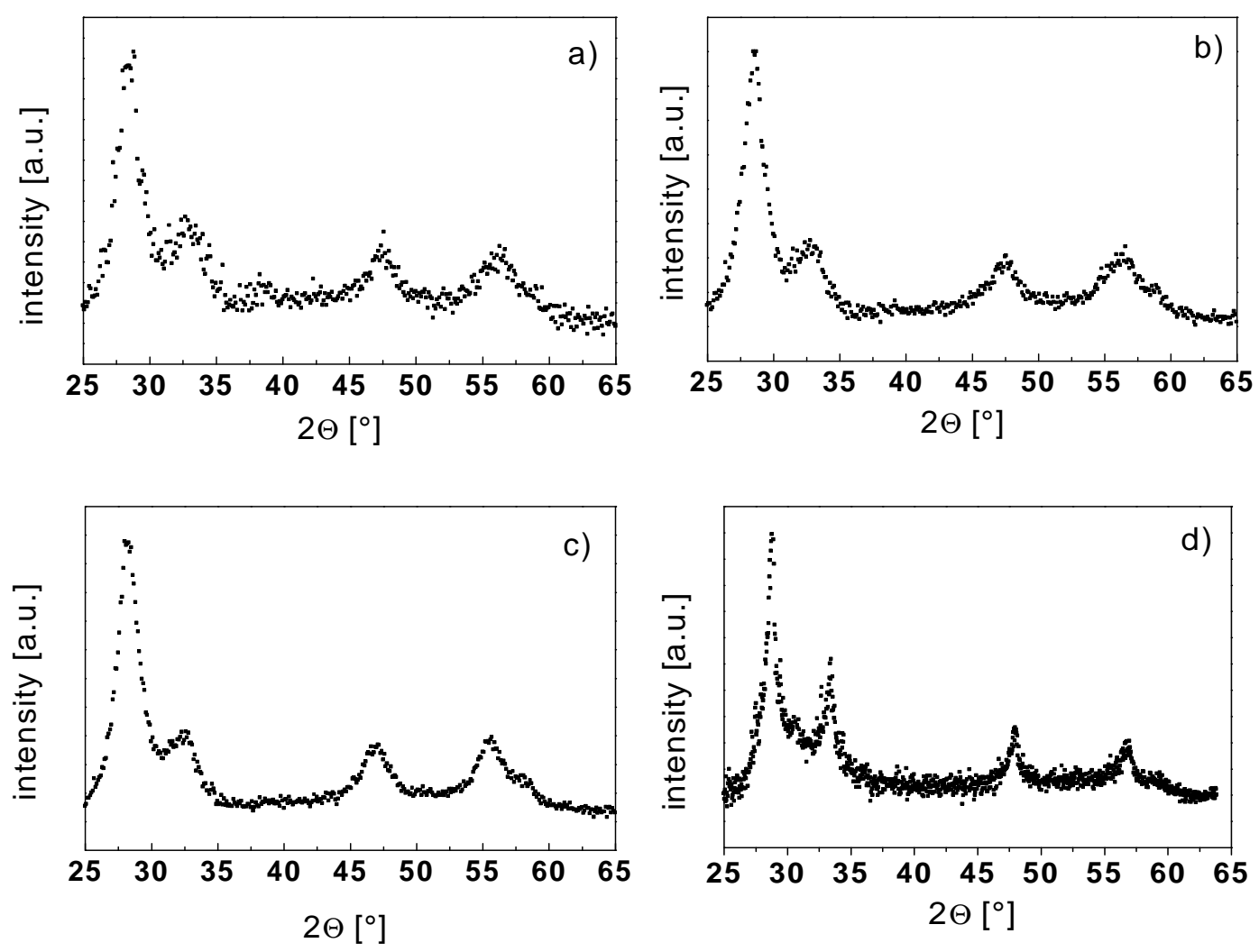

Figure 4. 1. Diffraction patterns of as-prepared Pd cluster samples at different current density $\left(I_{d}\right)$ prepared in $(90 \mathrm{ml}) 0.1 \mathrm{M} \mathrm{Oc}_{4} \mathrm{~N}^{+} \mathrm{Br}^{-}$in $\mathrm{CH}_{3} \mathrm{CN}$ at $D_{E}=3 \mathrm{~mm}, Q=720 \mathrm{~A} \cdot \mathrm{s}$ and $\mathrm{T}=22^{\circ} \mathrm{C}: \mathrm{I}_{d}=2.86$ $\mathrm{mA} / \mathrm{cm}^{2}(a), I_{d}=1.49 \mathrm{~mA} / \mathrm{cm}^{2}(b), I_{d}=0.57 \mathrm{~mA} / \mathrm{cm}^{2}(c)$ and $I_{d}=0.11 \mathrm{~mA} / \mathrm{cm}^{2}(d) . \lambda=1.120599 \AA$.

Table 4. 1. The effect of current density $I_{d}$ on the cluster size. The cluster size was determined by TEM and from XRD data using Scherrer formula.

\begin{tabular}{c|cc}
\hline$I_{d}$ & \multicolumn{2}{|c}{ cluster size $[\mathrm{nm}]$} \\
\cline { 2 - 3 }$\left[\mathrm{mA} / \mathrm{cm}^{2}\right]$ & $\mathrm{XRD}$ & TEM \\
5.71 & & $3.42 \pm 0.37$ \\
2.86 & 2.8 & $3.10 \pm 0.48$ \\
1.49 & 3.1 & $3.98 \pm 0.53$ \\
0.57 & 3.2 & $4.01 \pm 0.53$ \\
0.11 & 8.0 & $6.0 \pm 1.3$ \\
\hline
\end{tabular}



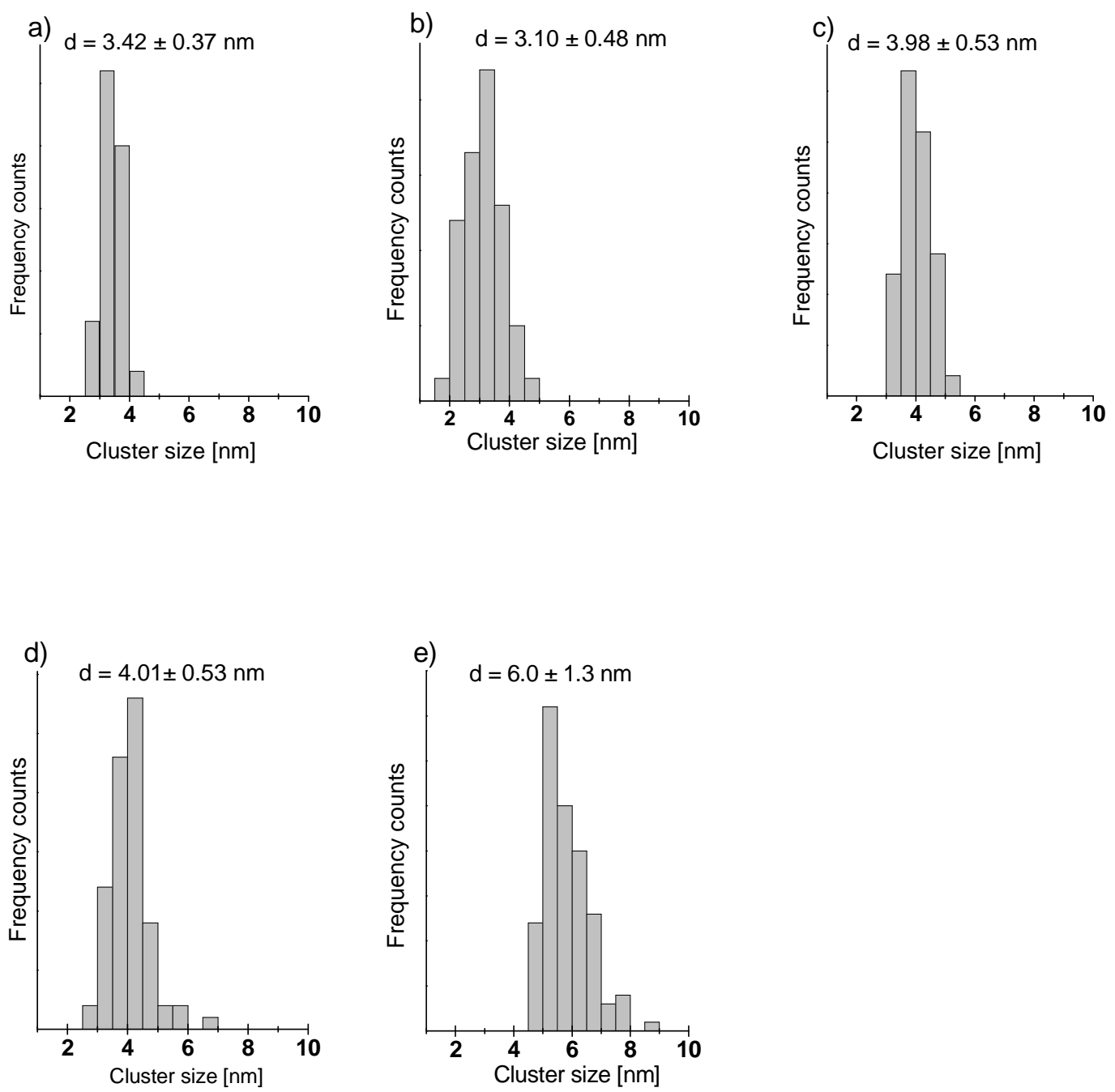

Figure 4. 2. Size distribution (TEM) of Pd clusters as a function of the current density $I_{d}\left(D_{E}=3 \mathrm{~mm}\right.$, $T=22^{\circ} \mathrm{C}, Q=720 \mathrm{~A} \cdot \mathrm{s}$, prepared in $(90 \mathrm{ml}) 0.1 \mathrm{M}$ of $\mathrm{Oc}_{4} \mathrm{~N}^{+} \mathrm{Br}^{-}$in $\left.\mathrm{CH}_{3} \mathrm{CN}\right): I_{d}=5.71 \mathrm{~mA} / \mathrm{cm}^{2}(a), I_{d}=$ $2.86 \mathrm{~mA} / \mathrm{cm}^{2}(b), I_{d}=1.49 \mathrm{~mA} / \mathrm{cm}^{2}(c), I_{d}=0.57 \mathrm{~mA} / \mathrm{cm}^{2}(d)$ and $I_{d}=0.11 \mathrm{~mA} / \mathrm{cm}^{2}(e)$. 


\subsubsection{Temperature Effect}

To study the effect of temperature, $\mathrm{T}$, on the size of $\mathrm{Pd}$ clusters the electrolyses were performed at $60{ }^{\circ} \mathrm{C}, 40{ }^{\circ} \mathrm{C}, 22{ }^{\circ} \mathrm{C}$ and $16{ }^{\circ} \mathrm{C}$. The distance between the electrodes was kept at $\mathrm{D}_{\mathrm{E}}=3 \mathrm{~mm}$, the current density was $2.86 \mathrm{~mA} / \mathrm{cm}^{2}$. All electrolysis runs were done in $(90 \mathrm{ml})$ $0.1 \mathrm{M} \mathrm{Oc}_{4} \mathrm{~N}^{+} \mathrm{Br}^{-}$and each run was stopped at $720 \mathrm{~A} \cdot \mathrm{s}$.

Isolation of the Pd clusters in solid form was performed by allowing the electrolyte to set for about 24 hours. A black precipitate was observed which was then recovered by siphoning off the clear solution. The precipitate was dried under high vacuum for ca. 3 hours. In the case of electrolysis at $16{ }^{\circ} \mathrm{C}$ no precipitate was formed although the $\mathrm{Pd}$ anode shows a loss of weight of $289 \mathrm{mg}$. Addition of de-aerated water forces the Pd-cluster to precipitate ${ }^{6}$ but the amount of the recovered cluster was very small (about 15\%).

The X-ray diffraction patterns of the as-prepared Pd cluster samples are shown in Figure 4.3 (a-c). Estimation of the cluster size $d$ from the full width at half the maximum (FWHM) of the main diffraction peak ((111) peak) shows that: $\mathrm{d}=2.58 \mathrm{~nm}$ at $60{ }^{\circ} \mathrm{C}(\mathrm{a}), \mathrm{d}=3.96 \mathrm{~nm}$ at $40^{\circ} \mathrm{C}$ (b), $\mathrm{d}=3.00 \mathrm{~nm}$ at $22^{\circ} \mathrm{C}$ (c) and $\mathrm{d}=2.17 \mathrm{~nm}$ at $16{ }^{\circ} \mathrm{C}$. The cluster size shows a maximum at $40{ }^{\circ} \mathrm{C}$. But, TEM analysis shows that the cluster size increases with increasing the temperature, Figure $4.4(\mathrm{a}-\mathrm{d}): \mathrm{d}=5.45 \pm 0.57 \mathrm{~nm}$ at $60{ }^{\circ} \mathrm{C}(\mathrm{a}), \mathrm{d}=4.23 \pm 0.36 \mathrm{~nm}$ at $40^{\circ} \mathrm{C}$ (b), $\mathrm{d}=3.10 \pm 0.48 \mathrm{~nm}$ at $22^{\circ} \mathrm{C}$ (c) and $\mathrm{d}=2.41 \pm 0.36 \mathrm{~nm}$ at $16{ }^{\circ} \mathrm{C}$ (e). One the other hand, the size distribution $\Delta \mathrm{d}$ is narrower with decreasing the Temperature: $\Delta \mathrm{d}= \pm 0.57 \mathrm{~nm}$ at $60{ }^{\circ} \mathrm{C}$ and $\Delta \mathrm{d}= \pm 0.36 \mathrm{~nm}$ at $16^{\circ} \mathrm{C}$.

The difference in the cluster size estimated from XRD data and TEM for the cluster obtained at $60{ }^{\circ} \mathrm{C}$ could not be due only to the difference in the estimation methods. Most probably this is due to a different cluster structure. This will be discussed in sections (5.3) \& (5.4).

\footnotetext{
${ }^{6}$ In this case a TEM sample of this electrolysis run was prepared by dipping the grid in the electrolyte.
} 

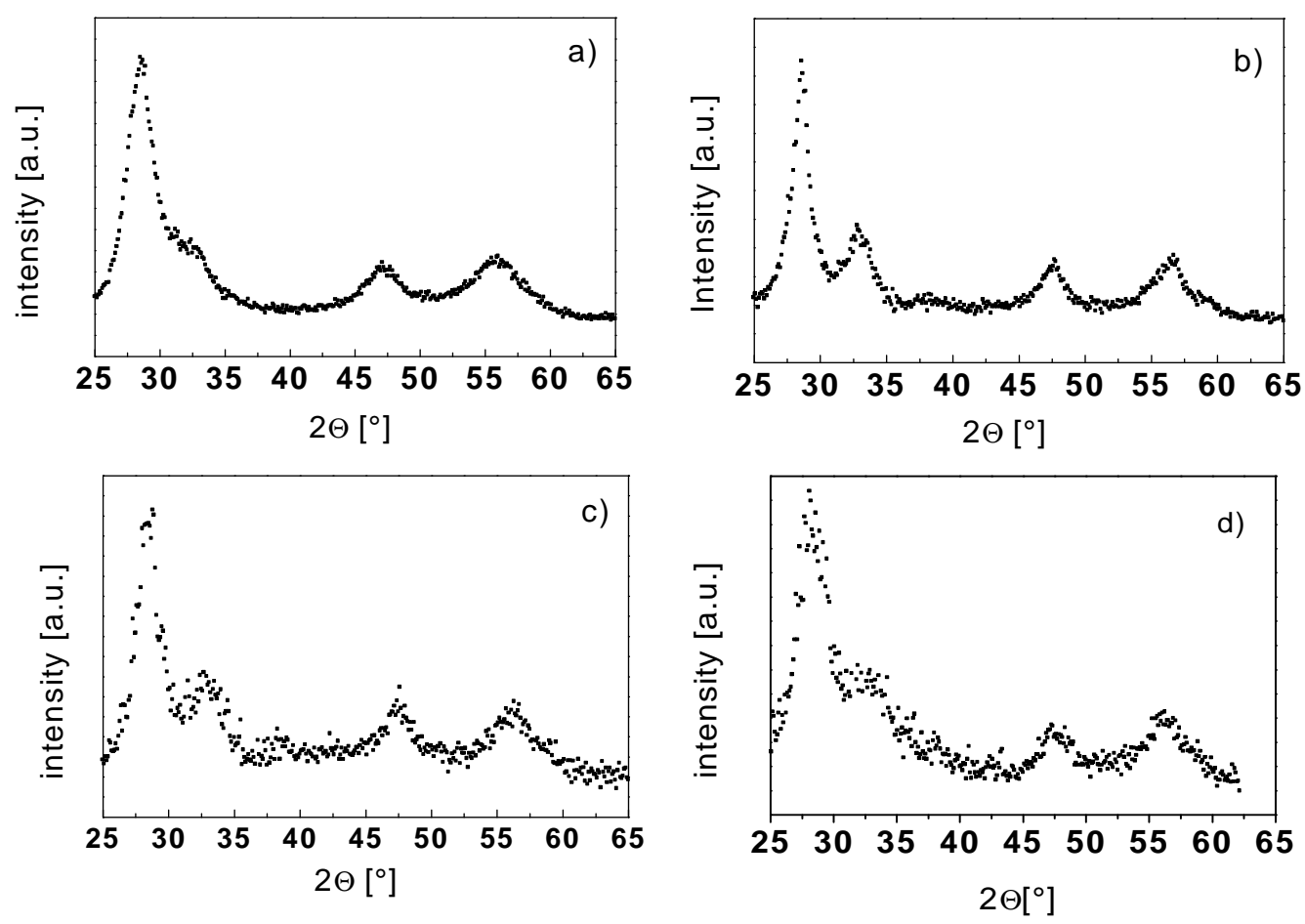

Figure 4. 3. Diffraction patterns of as-prepared Pd cluster samples prepared at different $T\left(D_{E}=3\right.$ $\mathrm{mm}, I_{d}=2.86 \mathrm{~mA} / \mathrm{cm}^{2}, Q=720 \mathrm{~A} \cdot \mathrm{s}$, prepared in $(90 \mathrm{ml}) 0.1 \mathrm{M}$ of $\mathrm{Oc}_{4} \mathrm{~N}^{+} \mathrm{Br}^{-}$in $\left.\mathrm{CH}_{3} \mathrm{CN}\right):($ a $) \mathrm{T}=60^{\circ} \mathrm{C}$, (b) $T=40^{\circ} \mathrm{C}$, (c) $\mathrm{T}=22^{\circ} \mathrm{C}$ and $(d) \mathrm{T}=16^{\circ} \mathrm{C} .(\lambda=1.120599 \AA)$.
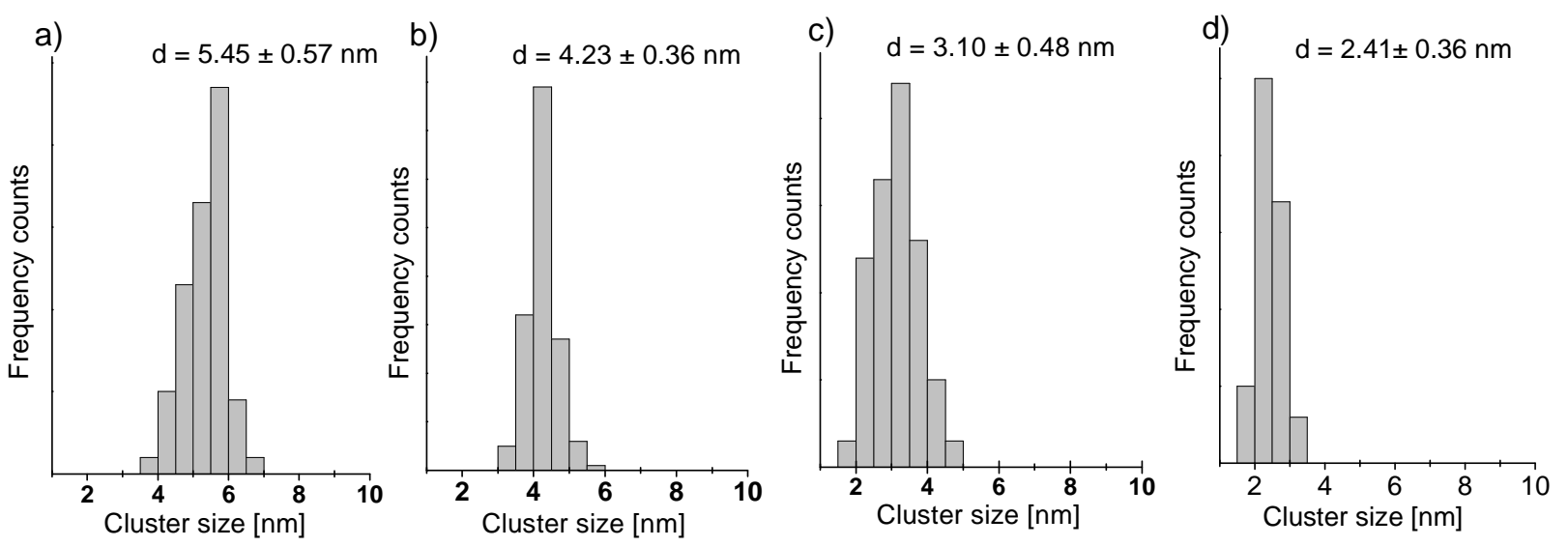

Figure 4. 4. Size distribution (TEM) of Pd clusters as a function of the $T\left(D_{E}=3 \mathrm{~mm}, I_{d}=2.86\right.$ $\mathrm{mA} / \mathrm{cm}^{2}, Q=720 \mathrm{~A} \cdot \mathrm{s}$, prepared in $(90 \mathrm{ml}) 0.1 \mathrm{M}$ of $\mathrm{Oc}_{4} \mathrm{~N}^{+} \mathrm{Br}^{-}$in $\left.\mathrm{CH}_{3} \mathrm{CN}\right): \mathrm{T}=60^{\circ} \mathrm{C}(\mathrm{a}), \mathrm{T}=40^{\circ} \mathrm{C}$ (b), $T=22{ }^{\circ} \mathrm{C}(c)$ and $T=16^{\circ} \mathrm{C}(d)$. 


\subsubsection{The Effect of the Distance between the Electrodes}

The effect of the distance between the anode and the cathode, $\mathrm{D}_{\mathrm{E}}$, was studied in four runs of electrolysis where the Pd anode and the cathode were placed $10.0 \mathrm{~mm}, 5.0 \mathrm{~mm}, 3.0 \mathrm{~mm}$ and $1.0 \mathrm{~mm}$ from each other. A current destiny of $2.86 \mathrm{~mA} / \mathrm{cm}^{2}$ was applied. The electrolysis was performed in (90ml) $0.1 \mathrm{M} \mathrm{Oc}_{4} \mathrm{~N}^{+} \mathrm{Br}^{-}$at $22{ }^{\circ} \mathrm{C}$ and stopped at $\mathrm{Q}=720 \mathrm{~A} \cdot \mathrm{s}$.

The X-ray diffraction patterns of as-prepared Pd cluster samples at different $\mathrm{D}_{\mathrm{E}}$ is shown in Figure 4.5 (a-d). Peak width analysis utilising the Scherrer formula shows that the cluster sizes are: $d=2.94 \mathrm{~nm}$ at $10 \mathrm{~mm}(\mathrm{a}), \mathrm{d}=3.23 \mathrm{~nm}$ at $5 \mathrm{~mm}(\mathrm{~b}), \mathrm{d}=3.00 \mathrm{~nm}$ at $3 \mathrm{~mm}$ (c) and $\mathrm{d}$ $=2.69 \mathrm{~nm}$ at $1 \mathrm{~mm}(\mathrm{~d})$. A maximum cluster size is observed at $5 \mathrm{~mm}$.

Figure 4.6 (a-d) shows TEM analysis of Pd clusters prepared at different $\mathrm{D}_{\mathrm{E}}$. Histograms (a) through (c) show that the cluster size decreases with decreasing the distance between the electrodes in the $D_{E}$ range of 10-3 mm, Figure $4.6(a-c): d=5.22 \mathrm{~nm}$ at $10 \mathrm{~mm}(\mathrm{a}), d=4.27$ $\mathrm{nm}$ at $5 \mathrm{~mm}$ (b) and $\mathrm{d}=3.10 \mathrm{~nm}$ at $3 \mathrm{~mm}(\mathrm{c})$, while electrolysis at $1 \mathrm{~mm}$ gives a cluster size of $4.15 \mathrm{~nm}$ (Fig.4.6(d)). Again, the difference between the TEM and the XRD results is occurring.

The size distribution is almost not affected by changing the distance between the electrodes. Electrolysis at different electrodes distances gives a constant size distribution width of about $\pm 0.50 \mathrm{~nm}$.

In these runs the clusters were isolated in solid form by allowing the electrolyte to stand for about 24 hour. The precipitate was then recovered and dried. 

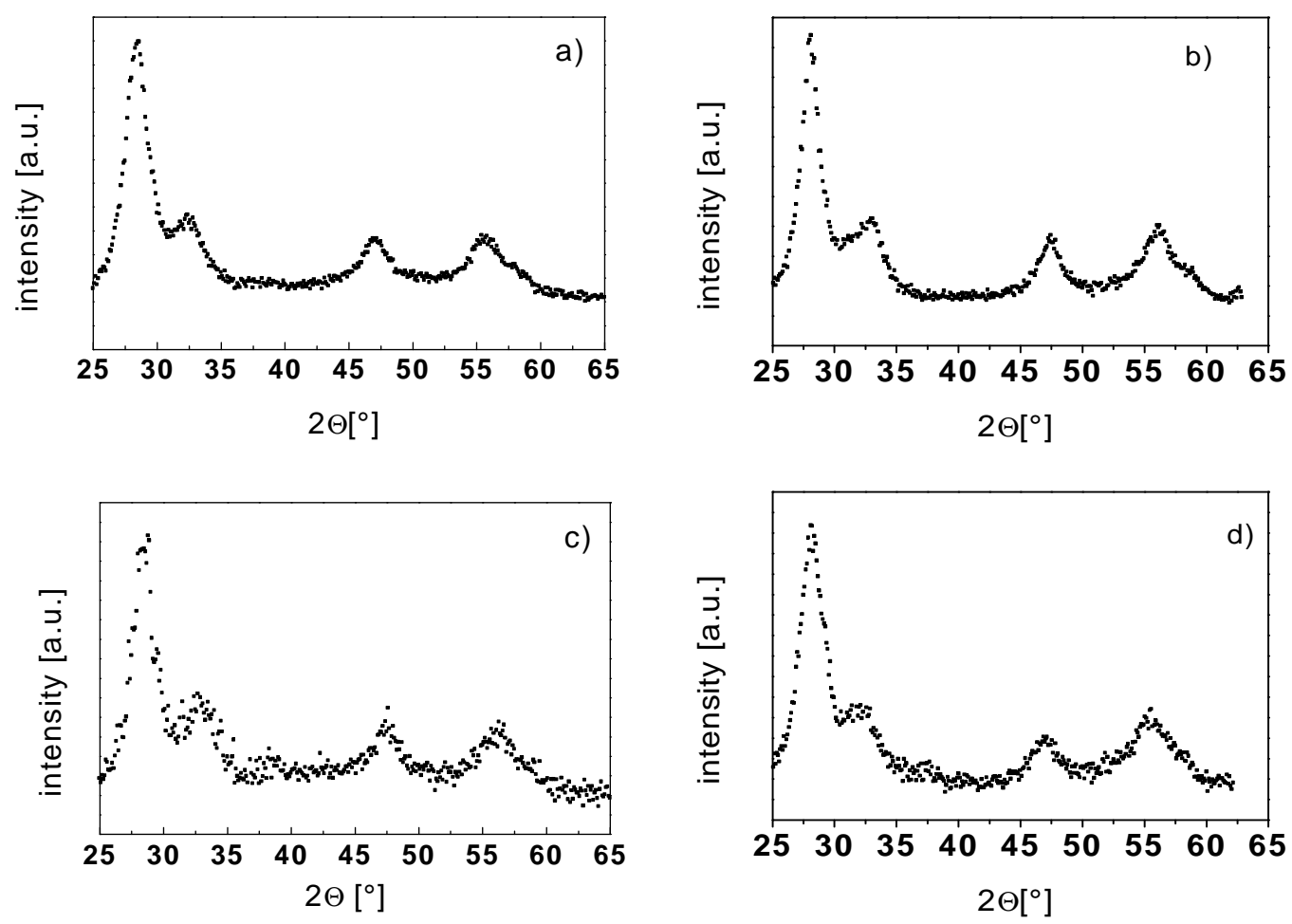

Figure 4. 5. Diffraction patterns of as-prepared $P d$ cluster samples prepared at different $D_{E}(T=$ $22^{\circ} \mathrm{C}, I_{d}=2.86 \mathrm{~mA} / \mathrm{cm}^{2}, Q=720 \mathrm{~A} \cdot \mathrm{s}$, prepared in $(90 \mathrm{ml}) 0.1 \mathrm{M} \mathrm{of} \mathrm{Oc}_{4} \mathrm{~N}^{+} \mathrm{Br}^{-}$in $\left.\mathrm{CH}_{3} \mathrm{CN}\right): D_{E}=10.0$ $\mathrm{mm}(\mathrm{a}), D_{E}=5.0 \mathrm{~mm}(b), D_{E}=3.0 \mathrm{~mm}(\mathrm{c})$ and $D_{E}=1.0 \mathrm{~mm}(\mathrm{~d}) .(\lambda=1.120599 \AA$. $)$
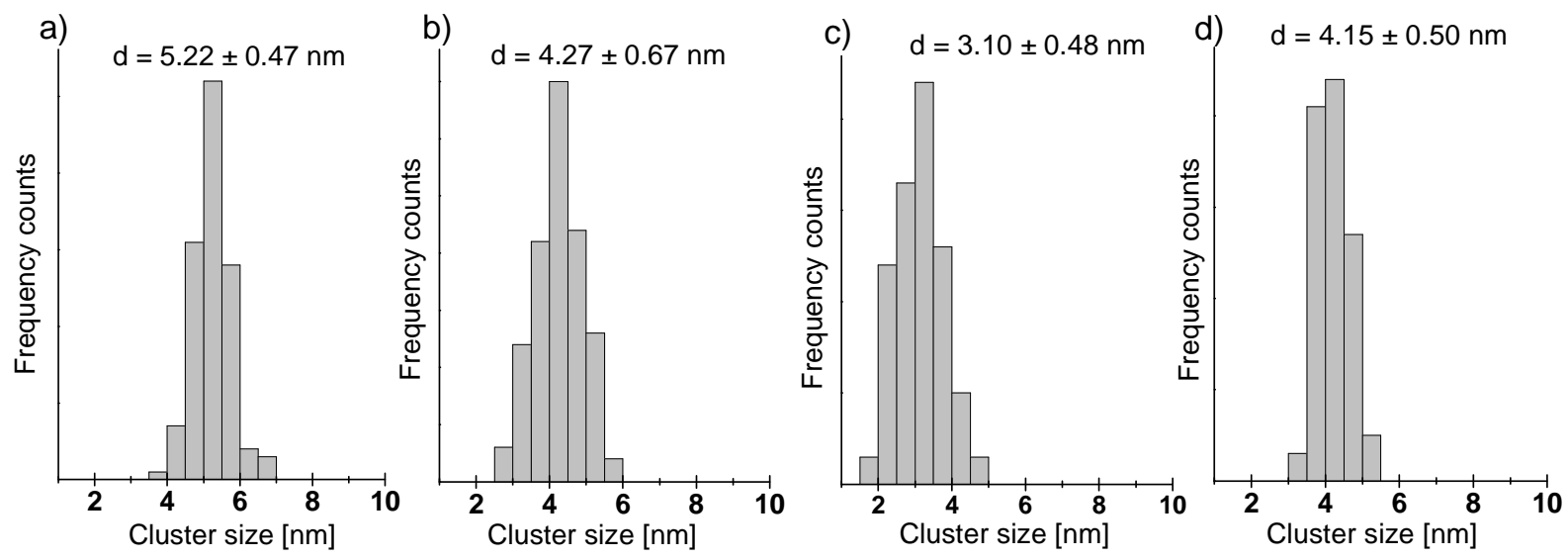

Figure 4. 6. Size distribution (TEM) of Pd clusters as a function of the $D_{E}\left(T=22{ }^{\circ} C, I_{d}=2.86\right.$ $\mathrm{mA} / \mathrm{cm}^{2}, Q=720 \mathrm{~A} \cdot \mathrm{s}$, prepared in $(90 \mathrm{ml}) 0.1 \mathrm{M} \mathrm{of} \mathrm{Oc} 4 \mathrm{~N}^{+} \mathrm{Br}^{-}$in $\left.\mathrm{CH}_{3} \mathrm{CN}\right): D_{E}=10.0 \mathrm{~mm}(\mathrm{a}), D_{E}=5.0$ $\mathrm{mm}(b), D_{E}=3.0 \mathrm{~mm}(c)$ and $D_{E}=1.0 \mathrm{~mm}(d)$. 


\subsubsection{Solvent Effect}

The possible influence of the solvent polarity was studied by using acetonitrle $\left(\mathrm{CH}_{3} \mathrm{CN}\right)$ tetrahydrofuran (THF) mixtures of precisely defined composition in which the percentage of $\mathrm{CH}_{3} \mathrm{CN}$ was varied from $100 \%$ to $0 \%$. In each run the time allowed for electrolysis corresponds to a charge flow $(\mathrm{Q})$ of $720 \mathrm{~A} \cdot \mathrm{s}$, the distance between the electrodes $\mathrm{D}_{\mathrm{E}}$ was 3 $\mathrm{mm}$ and the electrolysis temperature was $22^{\circ} \mathrm{C}$.

After the electrolysis was finished isolation of Pd clusters in a dry form was performed by slowly adding $20 \mathrm{ml}$ de-aerated water ${ }^{7}$ to electrolyte mixture with vigorously shaking it. The reaction mixture was allowed to stand for 24 hours where a precipitate was observed in the bottom of the flask and the reaction solution became clear. This was siphoned up by vacuum and the precipitate was then dried using a rotation pump for about 3 hours. In the case of electrolysis in $100 \%$ THF the recovery of the Pd cluster in a solid form was very poor (about $10 \%$ ) although weighting the $\mathrm{Pd}$ anode after the electrolysis shows that $209 \mathrm{mg} \mathrm{Pd}$ was dissolved corresponding to anode efficiency of $52.60 \%$.

The X-ray diffraction patterns of as-prepared samples in different electrolysis solvent is shown in Figure 4.7(a-e). Evaluation of the cluster size from XRD data by using the Scherrer formula shows that the cluster size decreases as the percentage of $\mathrm{CH}_{3} \mathrm{CN}$ decreases, Table 4.2. This means that the cluster size decreases as the solvent polarity decreases. This was also confirmed by TEM analysis shown in Figure 4.8 (a-f). On the one hand, it can be seen that the cluster size decreases with decreasing the solvent polarity. On the other hand, the size distribution becomes narrower as the solvent polarity decreases.

Table 4. 2. Effect of solvent on the cluster size. The cluster size was obtained from TEM and estimated from XRD data using the Scherrer formula.

\begin{tabular}{c|cccccc}
\hline cluster size & \multicolumn{7}{|c}{$\mathrm{CH}_{3} C N: T H F(v: v)$} \\
\cline { 2 - 7 }$[\mathrm{nm}]$ & $1: 0$ & $1: 1$ & $1: 2$ & $1: 3$ & $1: 4$ & $0: 1$ \\
XRD & 3.0 & 2.7 & 2.67 & 2.04 & 1.78 & \\
TEM & $3.10 \pm 0.48$ & $3.20 \pm 0.31$ & $3.08 \pm 0.47$ & $2.78 \pm 0.40$ & $2.88 \pm 0.31$ & $2.40 \pm 0.25$ \\
\hline
\end{tabular}

\footnotetext{
${ }^{7}$ In the run of electrolysis in $\mathrm{CH}_{3} \mathrm{CN}$ a precipitate was formed without adding de-aerated water.
} 

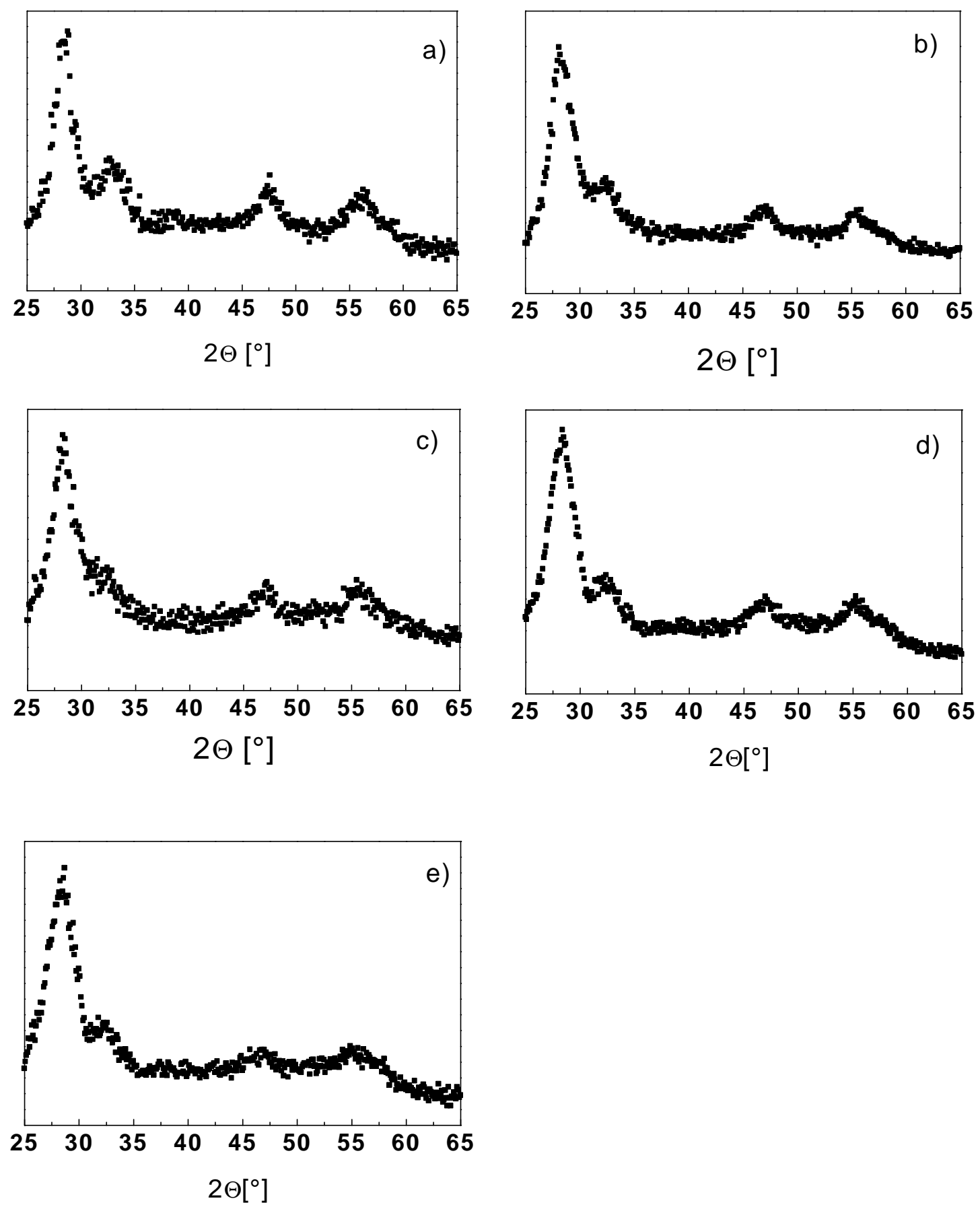

Figure 4. 7. Diffraction patterns of as-prepared Pd cluster samples prepared with different solvent

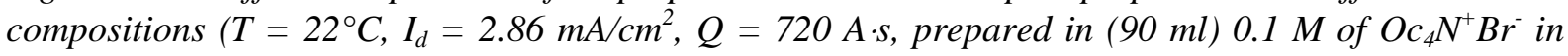
different $\mathrm{CH}_{3} \mathrm{CN}: \mathrm{THF}$ [v:v] mixtures): $\mathrm{CH}_{3} \mathrm{CN}: \mathrm{THF}=1: 0(a), \mathrm{CH}_{3} \mathrm{CN}: \mathrm{THF}=1: 1(b), \mathrm{CH}_{3} \mathrm{CN}: \mathrm{THF}$ $=1: 2(c), \mathrm{CH}_{3} \mathrm{CN}: \mathrm{THF}=1: 3(\mathrm{~d})$ and $\mathrm{CH}_{3} \mathrm{CN}: \mathrm{THF}=1: 4(\mathrm{e}) .(\lambda=1.120599 \AA$. $)$ 

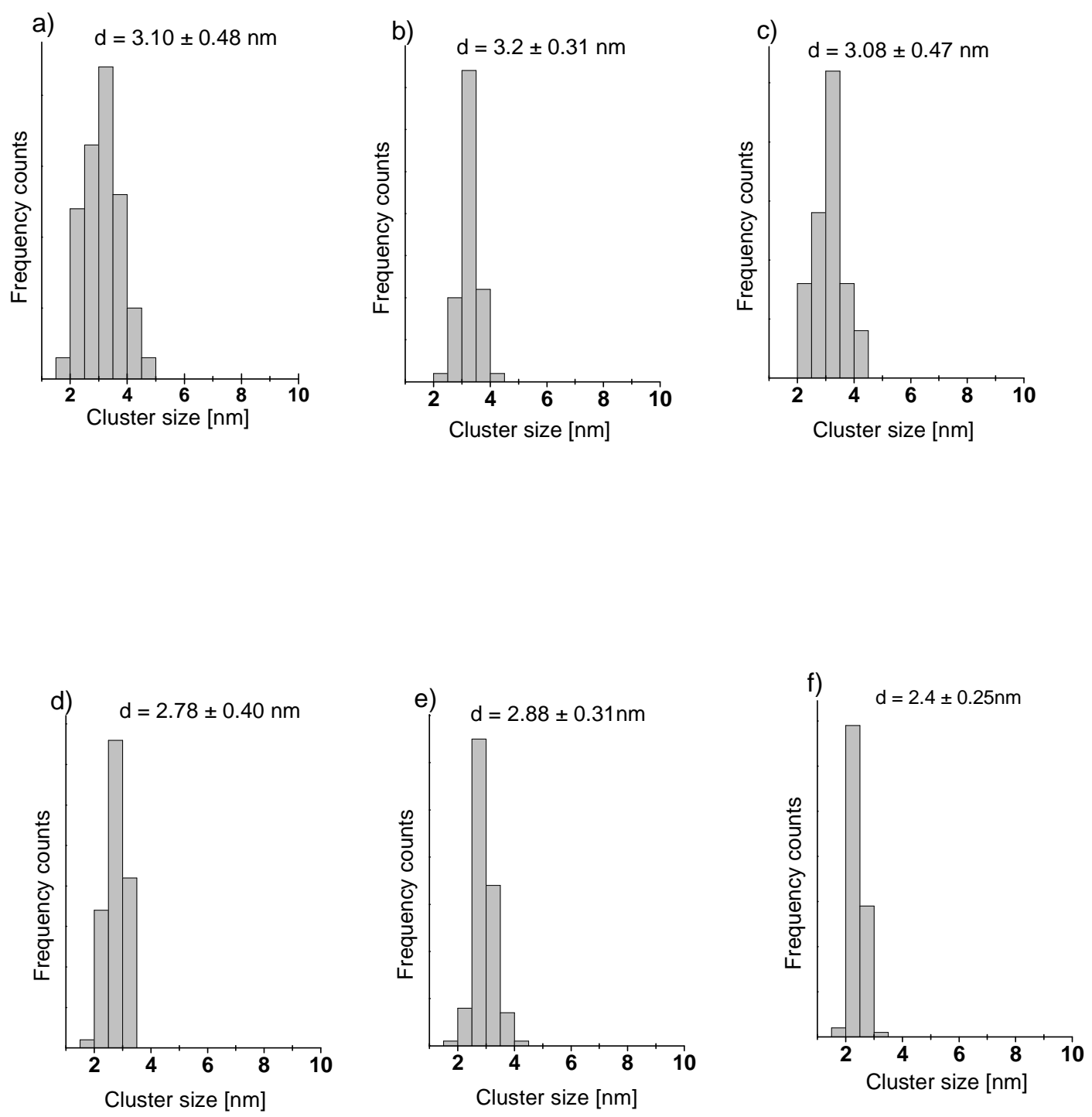

Figure 4. 8. Size distribution (TEM) of Pd cluster as a function of the solvent composition: $\left(T=22^{\circ} \mathrm{C}\right.$, $I_{d}=2.86 \mathrm{~mA} / \mathrm{cm}^{2}, Q=720 \mathrm{~A} \cdot \mathrm{s}$, prepared in $(90 \mathrm{ml}) 0.1 \mathrm{M} \mathrm{of} \mathrm{Oc}_{4} \mathrm{~N}^{+} \mathrm{Br}^{-}$in different $\mathrm{CH}_{3} \mathrm{CN}: \mathrm{THF}$ [v:v] ratio): $\mathrm{CH}_{3} \mathrm{CN}: \mathrm{THF}=1: 0(\mathrm{a}), \mathrm{CH}_{3} \mathrm{CN}: \mathrm{THF}=1: 1(\mathrm{~b}), \mathrm{CH}_{3} \mathrm{CN}: \mathrm{THF}=1: 2$ (c), $\mathrm{CH}_{3} \mathrm{CN}: \mathrm{THF}=1: 3$ (d), $\mathrm{CH}_{3} \mathrm{CN}: \mathrm{THF}=1: 4(e)$ and $\mathrm{CH}_{3} \mathrm{CN}: \mathrm{THF} 0: 1(f)$. 


\section{Discussion: Pd Clusters Preparation}

In this chapter the experimental results on the Pd cluster preparation, presented in the previous chapter, will be discussed. Explanation for the influence of the electrolyses parameters on the cluster size and on the size distribution will be viewed. A model describing the thickness of the stabilisation shell will be confirmed. Discussing various methods to determine the size and the structure of the cluster will be presented. The effect of the cluster structure on estimating the cluster size from XRD data using peak width analysis will be discussed.

\subsection{Influence of the electrolysis parameters}

Size selective synthesis is one of the most attractive goals that a synthetic chemist tries to achieve. In the previous chapter we have seen that the cluster size can be controlled by a variety of preparation parameters including the current density, the temperature, the solvent and even the distance between the electrodes. In the following the influence of these parameters will be discussed. To smooth the discussion, part of the results from chapter 4 will be represented graphically.

\subsubsection{Current density effect}

In this work we have found that increasing the current density decreases the Pd clusters size, Figure 5.1. As have been shown in section (2.5), in electrochemical powder productions the critical radius $r_{\text {crit. }}$ of the cluster of ad-atoms prior to powder formation at the cathode interface is predicted by Eqn. (2.11)

$$
r_{\text {crit. }}=\frac{2 M \gamma}{n F \eta \rho}
$$


In this work Pd clusters in the range of 3.0 to $6.0 \mathrm{~nm}$ (TEM) could be selectively prepared depending on the applied current density value, Figure 5.1.

The cathodic overpotential during the electrolysis was not determined in this study, for this reason a direct comparison between the imposed potential and the theory is not possible. However, for large current densities the overpotential $\eta$ is proportional to logarithm of $I_{d}$ according to [73]:

$$
\eta=\frac{R T}{\propto n F} \cdot \ln \frac{I_{d}}{I_{o}}
$$

where, $I_{o}$ is the exchange current density and $\propto$ is a transfer coefficient of about 0.5 . Inserting Eqn. (5.1) into Eqn. (2.11) and rearranging yields:

$$
\frac{1}{d}=\frac{1}{2 r_{c r i t}}=\frac{R T \rho}{4 M \gamma \propto} \cdot \ln \frac{I_{d}}{I_{o}}
$$

The values obtained in this study were plotted in Fig. 5.1 as the reciprocal cluster diameter versus $\ln I_{d}$. A least square fit to the data yields a slope of $0.035 \mathrm{~nm}^{-1}$. According to Eqn. (5.2) this value corresponds to a surface energy of $\gamma=4 \mathrm{~J} / \mathrm{m}^{2}$ for $\propto=0.5$. As the value for $\gamma$ has the right order of magnitude, it is concluded that the theory presented in section (2.5) explains the experimental findings and that the results of this study are in agreement with the model of Turkevich [61]. 


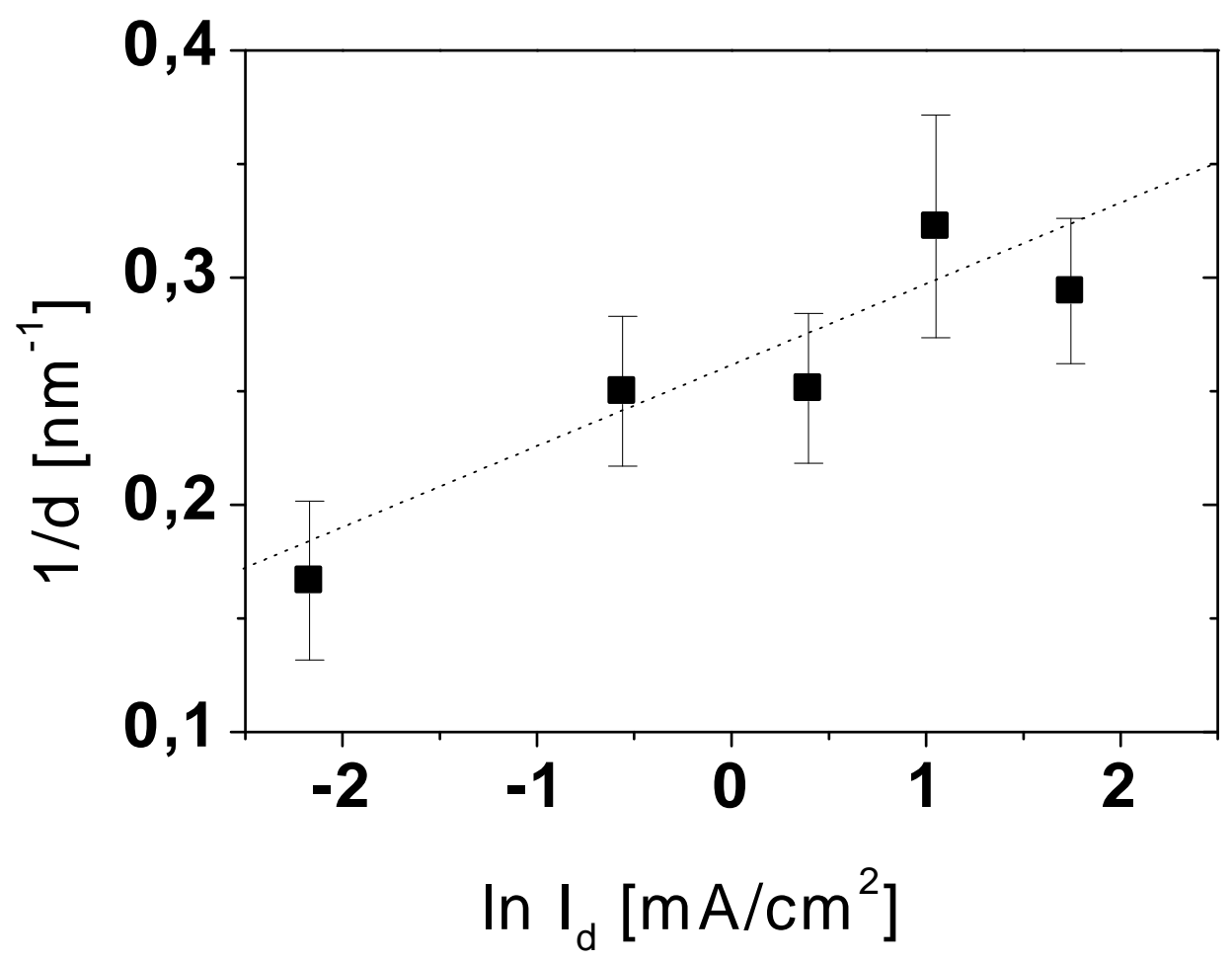

Figure 5. 1. The effect of the current density on the size of the prepared Pd cluster obtained by TEM plotted according to Eqn. (5.2). All samples were prepared in $(90 \mathrm{ml}) 0.1 \mathrm{M} \mathrm{Oc}_{4} \mathrm{~N}^{+} \mathrm{Br}^{-}$in $\mathrm{CH}_{3} \mathrm{CN}$ at $\mathrm{D}_{E}$ $=3 \mathrm{~mm}, Q=720 \mathrm{~A} \cdot \mathrm{s}$ and at $22^{\circ} \mathrm{C}$. The error bars represents the size distribution of each cluster sample. 


\subsubsection{Temperature effect}

Increasing the temperature of the electrolysis increases the cluster size, as summarised in Figure 5.2. A selective preparation of Pd clusters in the range of 2.4 to $5.5 \mathrm{~nm}$ (TEM) can be obtained depending on the temperature used for electrolysis.

These results can, in one hand, be explained by postulating higher diffusion, migration and dissociation rate of the tertraoctylammonium palladate $\left[\left(\mathrm{Oc}_{4} \mathrm{~N}^{+}\right)_{2} \mathrm{Pd}^{2-}\right]$ intermediate at higher temperatures and, on the other hand, decreasing the viscosity of the medium leading to low the cathodic overpotentials [75].

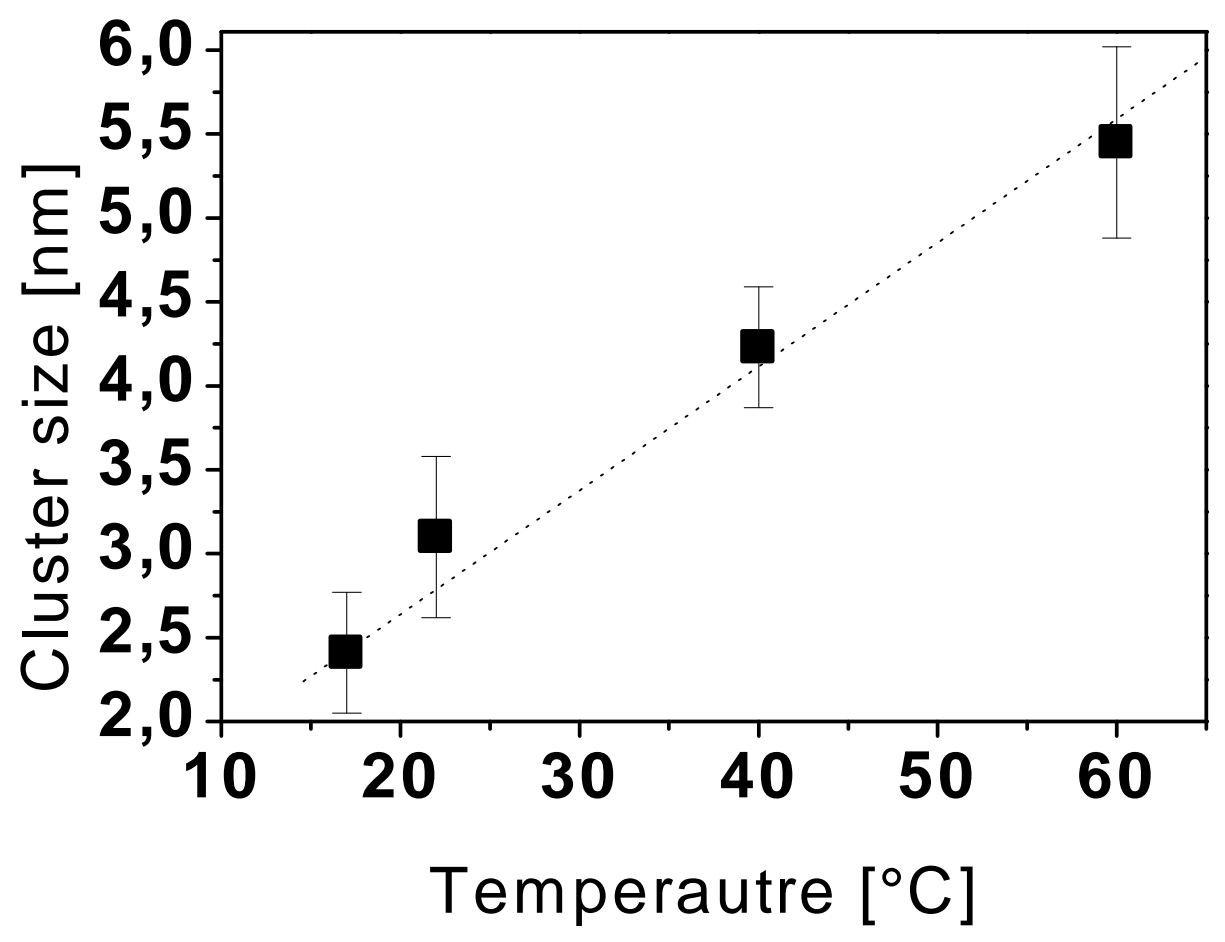

Figure 5. 2. The effect of the temperature on the Pd cluster size obtained by TEM analysis: $\left(D_{E}=3\right.$

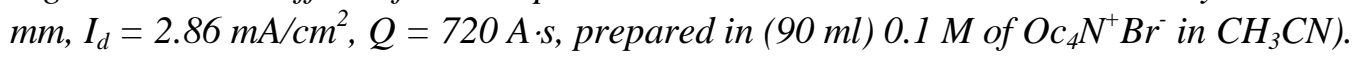




\subsubsection{The effect of the distance between the electrodes $D_{E}$}

Another factor which was found to affect the Pd cluster size is the distance between the electrodes $\mathrm{D}_{\mathrm{E}}$. Cluster sizes in the range of 3.1 to $5.2 \mathrm{~nm}$ (TEM) can be prepared depending on the selected distance between the Pd anode and the cathode, Figure 5.3.

Similar results in the $\mathrm{D}_{\mathrm{E}}$ range 1-3 mm were reported by Reetz et al. where it was suggested that a variety of factors are probably involved, like transport rates and electrophoretic mobility [75]. Details are not clear yet.

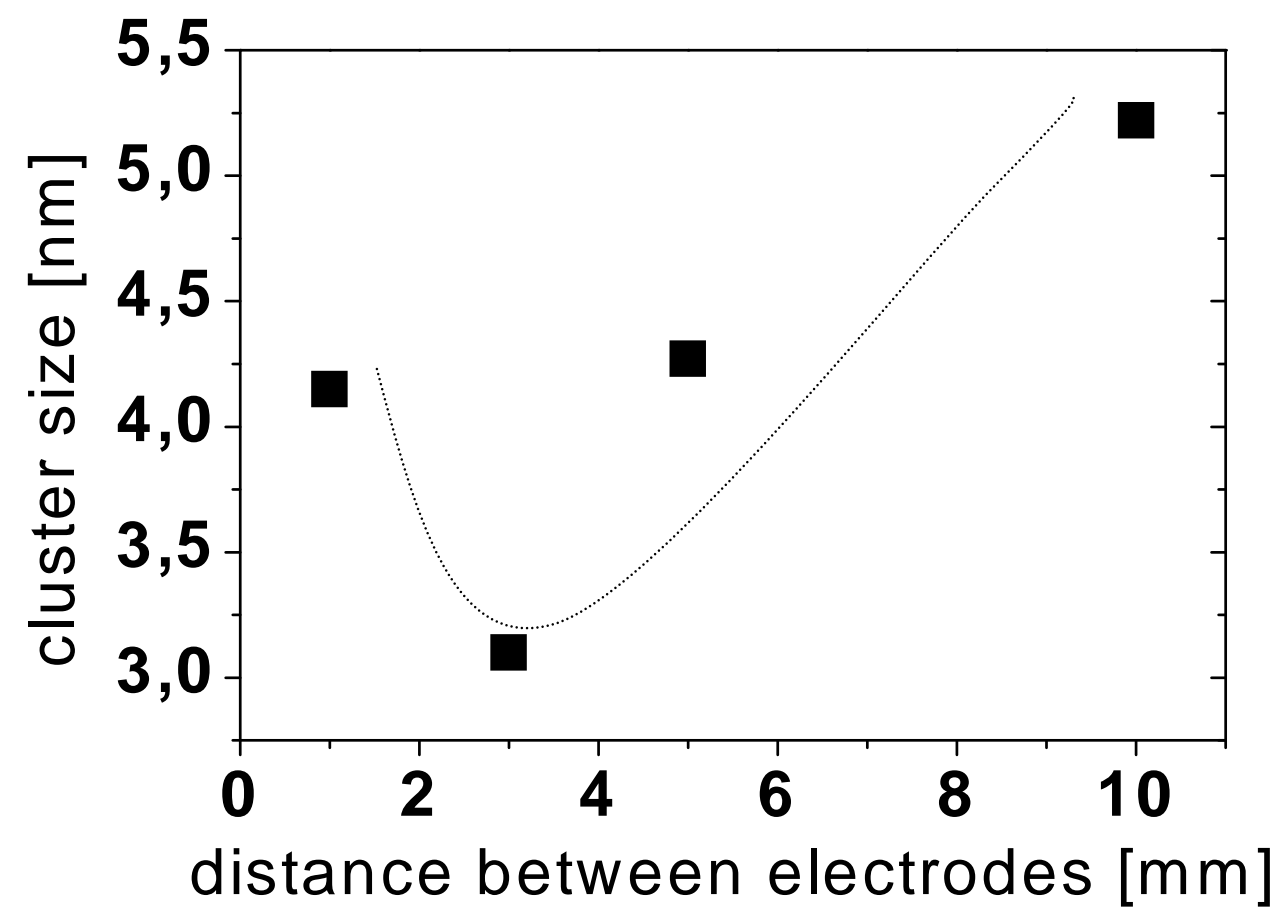

Figure 5. 3. Influence of $D_{E}$ on Pd clusters size (TEM) prepared in $(90 \mathrm{ml}) 0.1 \mathrm{Mof} O \mathrm{Oc}_{4} \mathrm{~N}^{+} \mathrm{Br}$ in $\mathrm{CH}_{3} \mathrm{CN}, \mathrm{T}=22^{\circ} \mathrm{C}, I_{d}=2.86 \mathrm{~mA} / \mathrm{cm}^{2}, Q=720 \mathrm{~A} \cdot \mathrm{s}$. 


\subsubsection{The solvent effect}

Electrolysis in different solvents gives Pd cluster sizes in the range between $3.10 \mathrm{~nm}$ (TEM) in pure $\mathrm{CH}_{3} \mathrm{CN}$ and $2.40 \mathrm{~nm}$ (TEM) in pure THF, as summarised in Figure 5.4.

$\mathrm{CH}_{3} \mathrm{CN}$ is a very polar solvent with a solvent polarity parameter of $\mathrm{E}_{\mathrm{T}}=45.6$, whereas THF is considerably less polar with $\mathrm{E}_{\mathrm{T}}=37.4$ [76].

In good agreement, Helbig [74] has also found that increasing the polarity of the medium the average size of the Pd cluster increases, the range stretching from $1.4 \mathrm{~nm}$ in pure THF to 4.8 $\mathrm{nm}$ in a 1:1 mixture of THF and $\mathrm{CH}_{3} \mathrm{CN}$. This was explained by enhancement of the growth as the polarity increased. A reduced electrostatic interaction between the metal nano-particles and the $\mathrm{Oc}_{4} \mathrm{~N}^{+} \mathrm{Br}^{-}$in a polar surrounding could be a possible explanation for this observation. In a less polar solvent the stabilising shell would then more effectively shield the nanoparticle against further growth.

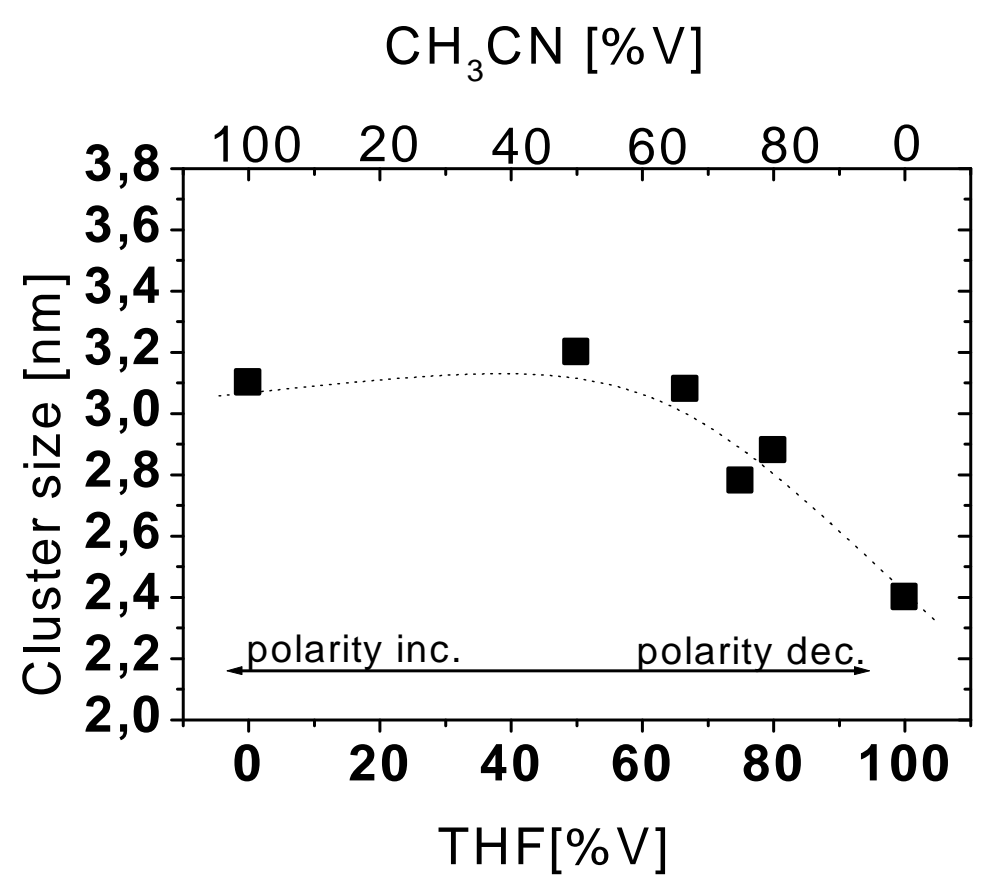

Figure 5. 4. The effect of the solvent on the Pd cluster size prepared at $T=22^{\circ} \mathrm{C}, I_{d}=2.86 \mathrm{~mA} / \mathrm{cm}^{2}, Q$ $=720 \mathrm{~A} \cdot \mathrm{s}$ in $(90 \mathrm{ml}) 0.1 \mathrm{M} \mathrm{Oc}_{4} \mathrm{~N}^{+} \mathrm{Br}$ using different mixtures of $\mathrm{THF}$ and $\mathrm{CH}_{3} \mathrm{CN}$. 


\subsection{Thickness of the stabilisation shell}

In this work the $\mathrm{Pd}$ nano-particles are stabilised by the adsorption of $\mathrm{Oc}_{4} \mathrm{~N}^{+} \mathrm{Br}^{-}$at their surface, thus providing a protective layer or a stabilisation shell, Figure 5.5. Reetz and coworkers [77] determine the thickness of the stabilisation shell for a series of palladium clusters stabilised by tetraalkylammonium salts $\left(\mathrm{R}_{4} \mathrm{~N}^{+} \mathrm{X}^{-}\right)$, where a combined STM-TEM study revealed that each individual cluster is surrounded by one monomolecular layer of $\mathrm{R}_{4} \mathrm{~N}^{+} \mathrm{X}^{-}$.

In this work HREM images demonstrate that the distance between individual clusters was found to be about $2 \mathrm{~nm}$, Figure 5.6. This indicates that the thickness of the stabilisation shell is $1 \mathrm{~nm}$, which means that the Pd cluster is stabilised with one monomolecular layer of $\mathrm{Oc}_{4} \mathrm{~N}^{+} \mathrm{Br}^{-}$(chain length is about $1.1 \mathrm{~nm}$ ), which is in accordance with the finding of Reetz and co-workers. 

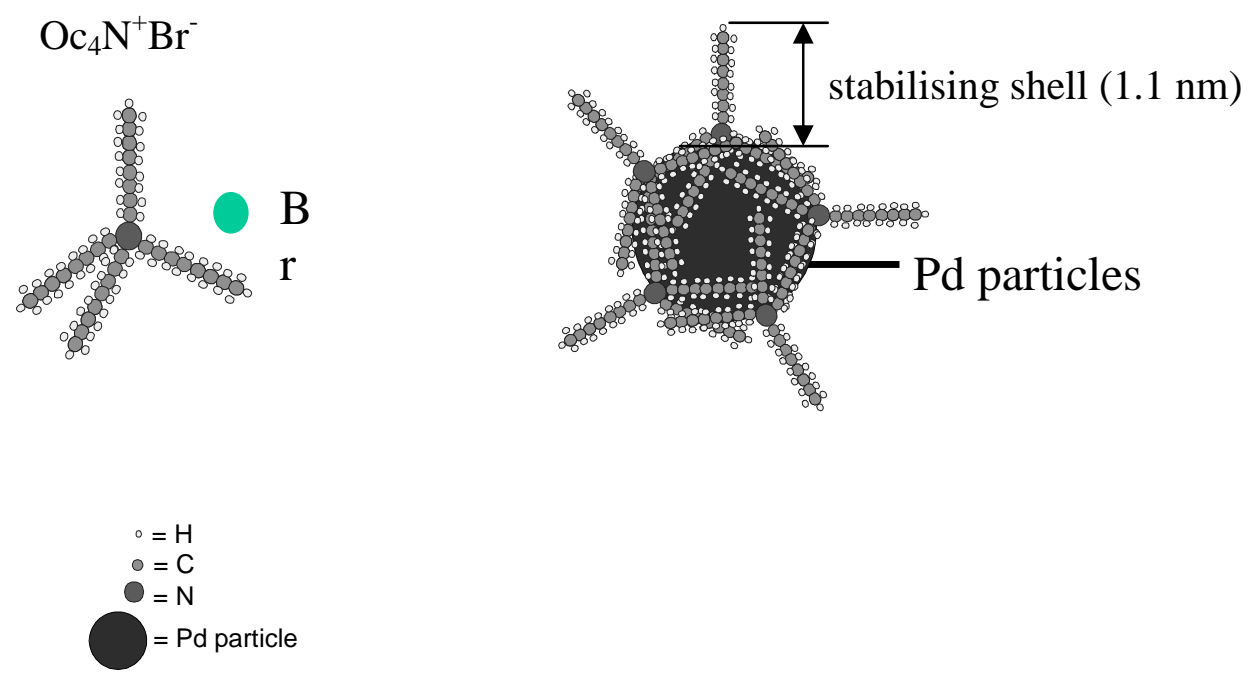

Figure 5. 5. Model for the stabilisation of $\mathrm{Pd}$ cluster by monomolecular layer of $\mathrm{Oc}_{4} \mathrm{~N}^{+} \mathrm{Br}$.

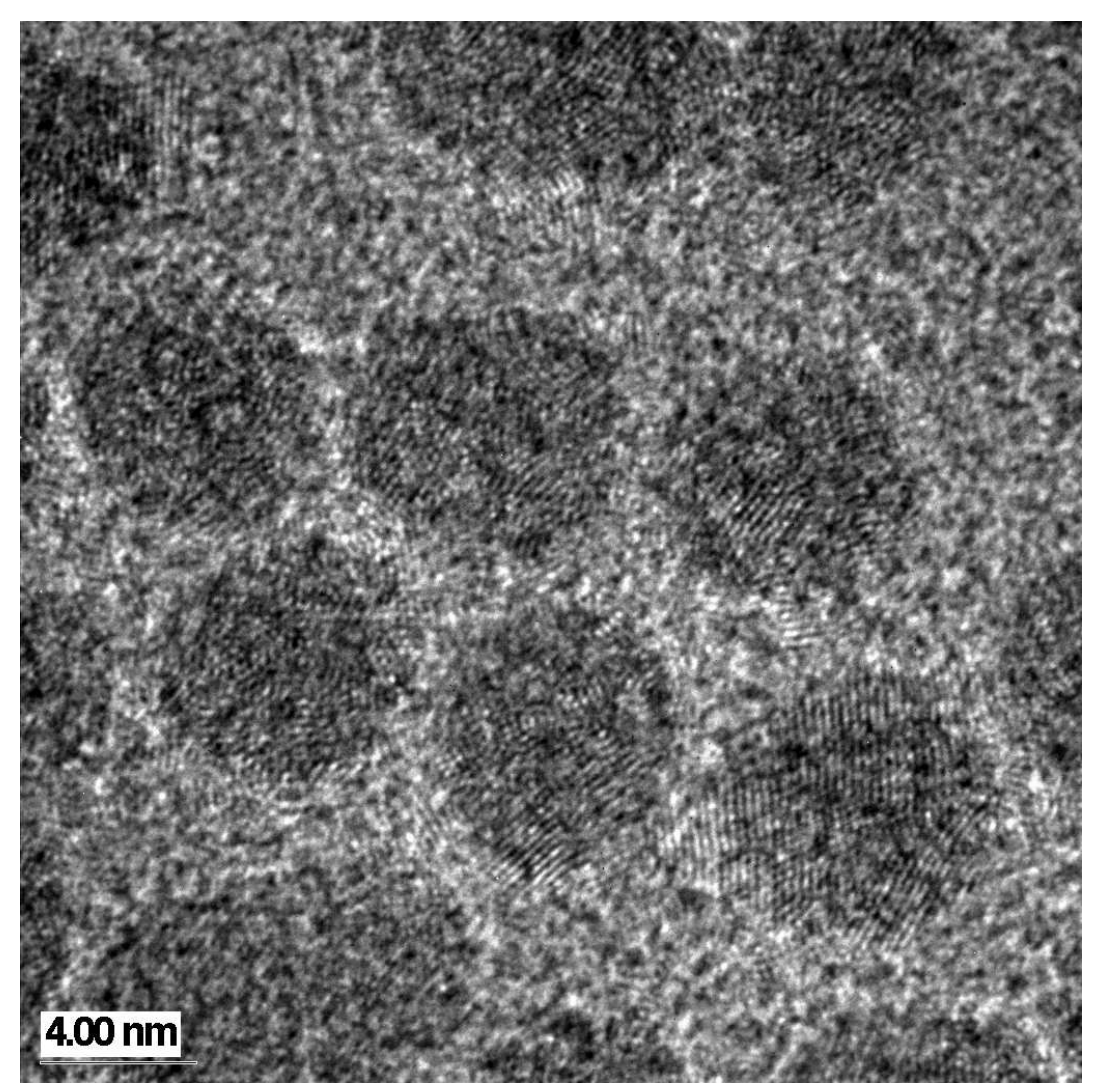

Figure 5. 6. HREM image of Pd cluster ( sample ms302) showing a distance of about $2 \mathrm{~nm}$ between each individual cluster 


\subsection{Cluster size determination}

In this section determination of the cluster size will be discussed using XRD data and TEM. The estimation of the cluster size using XRD data will be done by two methods: First by using the Scherrer formula, and second by taking a Fourier transform of a diffraction pattern. This will be compared with the size obtained for a model Pd cluster in two different lattice structures.

In chapter 4 we have seen that the cluster size, for the same sample, determined by the Scherrer formula differs from the size determined by TEM. This problem arises because of the variety of structures and the size dependent nature of the diffraction pattern [78]. Difficulties in applying the peak-width based analysis to clusters have been reported by a number of workers $[79,80]$. The small size as well as the mechanical stress in clusters leads to peak broadening [81]. There are indications that the outermost shells of an icosahedral cluster are closer to the second most outer shell than all the other inter-shell distance [82]. This means that the Scherrer formula is not accurate in estimating the cluster size. This formula is derived by assuming that the diffraction peak is associated with a family of crystal planes in a size-limited crystal. The existence of the so-called multiply twinned particles (MTP) and icosahedral particles, or even the high proportion of atoms at the surface of aggregates means that transitional symmetry is not an underlying feature of clusters. To associate diffraction features with the notion of Bragg peaks from a family of crystal planes is not appropriate in this case. Diffraction patterns are better thought of as a superposition of continuous oscillatory function in reciprocal space [78].

In this work we have found that estimations of the Pd cluster size from XRD data using Scherrer formula for Pd cluster samples, in most cases, gives values smaller than those obtained by TEM method, Table 5.2. We attribute this difference in the cluster size for the same sample obtained by the two methods to an icosahedral structure of the Pd clusters. The presence of an icosahedral structure will lead to peak broadening which means that estimations of the size of an icosahedral cluster using the XRD data will give a smaller value than the real size. In the following this will be demonstrated by a model cluster. 
Table 5. 1. Comparison of the Pd cluster size [nm] for different samples obtained from XRD data by using Scherrer formula and TEM.

\begin{tabular}{c|cc}
\hline Sample No. & \multicolumn{2}{|c}{ Cluster size [nm] } \\
& XRD & TEM \\
\hline msE13 & 2.0 & $3.0 \pm 0.5$ \\
ms615 & 2.2 & $3.6 \pm 0.4$ \\
ms302 & 3.5 & $5.0 \pm 0.6$ \\
ms718 & 3.4 & $4.8 \pm 0.8$ \\
ms706 & 4.6 & $5.2 \pm 0.6$ \\
msE21 & 6.0 & $5.6 \pm 1.1$ \\
ms717 & 8.0 & $6.0 \pm 1.3$ \\
\hline
\end{tabular}

Figure 5.7 shows the XRD patterns for a model Pd cluster (2057 atom about $3.7 \mathrm{~nm}$ ) obtained by MD-simulation from two different lattices, (a) cubic and (b) icosahedral, [83]. It is clearly visible that the icosahedral cluster has broader peaks than those for the cubic one. Estimation of the cluster size for the peak width ((111) peak) using the Scherrer formula gives a size of $3.6 \mathrm{~nm}$ for the cubic cluster and $2.2 \mathrm{~nm}$ for the icosahedral cluster. This difference is not real and it is due to peaks broadening resulting from the strained icosahedral structure compared to the cubic structure.

Recently a simple approach to estimate the size of gold clusters was presented by Hall [78], in which a Fourier transform of the X-ray diffraction pattern was taken. By doing that, information related to the average distribution of the interatomic distances within the cluster is obtained. The last visible peak of the radial distribution function (r.d.f) or P(r) gives a fairly accurate value of the sample size. In this work we have applied this technique to the two types of diffraction data: model cluster and three experimental diffractograms of Pd cluster, for which independent TEM observations have been made.

Figure 5.8 shows the Fourier transform of the diffraction patterns of the model cluster shown in Figure 5.7. In both icosahedral and cubic structures there is a trend of decreasing oscillation in the $\mathrm{P}(\mathrm{r})$ with increasing the interatomic distance. In both cases the oscillation vanishes at an interatomic distance of $3.7 \mathrm{~nm}$ which is also the size of this (2057 atoms) model cluster. 


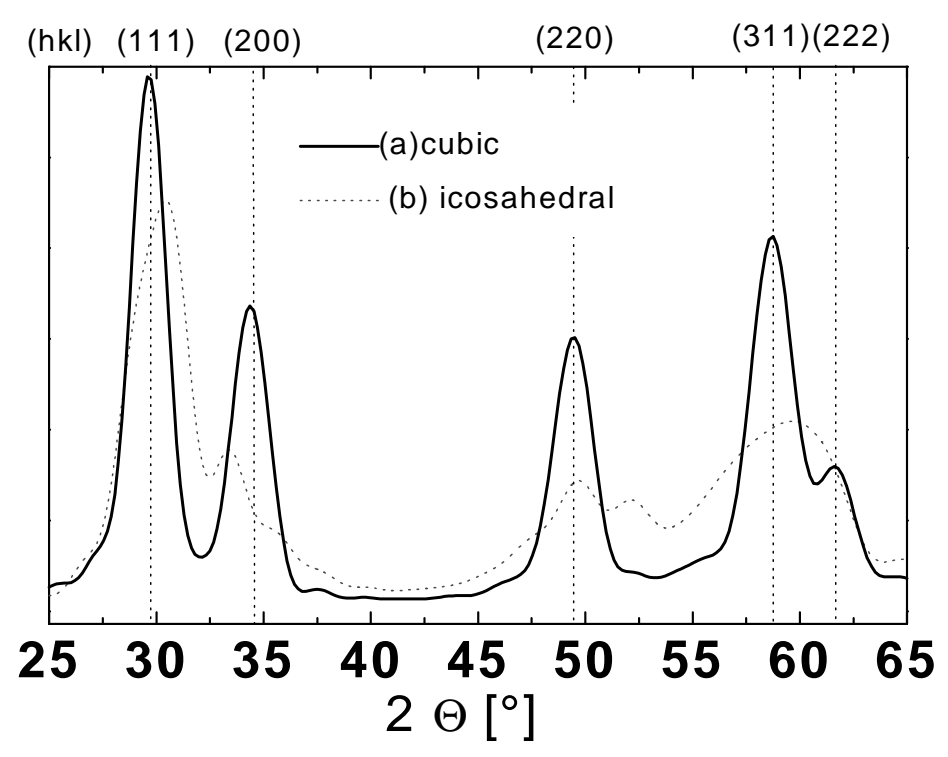

Figure 5. 7. Diffraction patterns of a model Pd clusters:(a) shows the diffraction patterns of a cubic Pd cluster (2057 atoms about $3.7 \mathrm{~nm}$ ), (b) represents an icosahedral Pd cluster (2057 atoms about 3.7 $\mathrm{nm}$ ). The dotted vertical lines are the position of the Bragg reflections for bulk Pd with cubic lattice structure after [83].

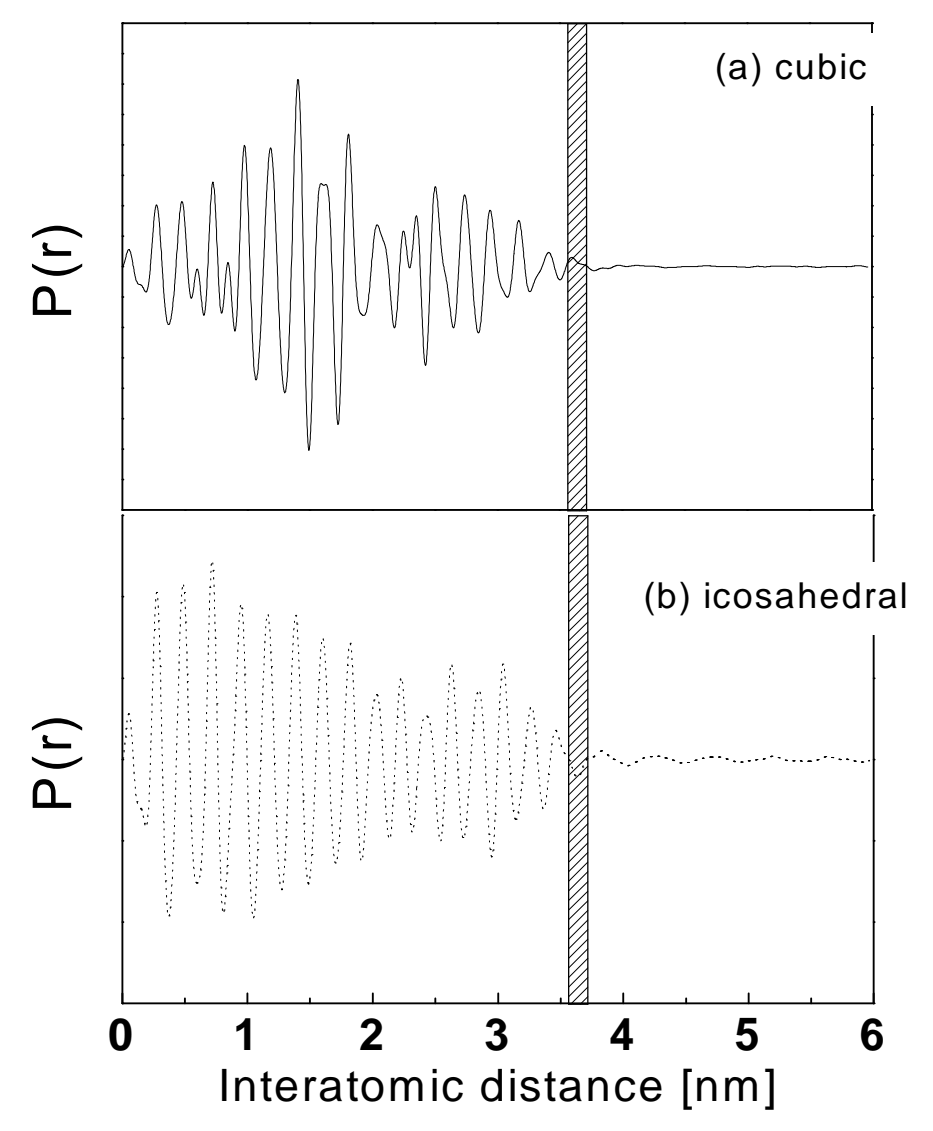

Figure 5. 8. P(r) for a model Pd cluster (a) icosahedral,b) cubic. In both cases the P(r) oscillations vanish at interatomic distance of about $3.7 \mathrm{~nm}$. 
Figure 5.9(a-c), shows the diffraction patterns of three different Pd cluster samples. The cluster size obtained from the application of the Scherrer formula are: (a) $6.0 \mathrm{~nm}$, (b) $3.5 \mathrm{~nm}$ and (c) $2.2 \mathrm{~nm}$.

Figure 5.9 (a-c) shows, also, the TEM analysis of these samples. One obtains: (a) $5.6 \pm 1.1$ $\mathrm{nm}$, (b) $5.0 \pm 0.6 \mathrm{~nm}$ and (c) $3.6 \pm 0.4 \mathrm{~nm}$. These values are different than those obtained from XRD data. This difference is attributed to different cluster structures of the samples. This will be confirmed in the next section (5.4). The smaller XRD size values obtained in (b) $(3.5 \mathrm{~nm}$ from (XRD) $5.0 \mathrm{~nm}$ from (TEM)) and in (c) (2.2 nm from (XRD) and $3.6 \mathrm{~nm}$ from (TEM)) are due to the iscoahedral structure of these cluster samples. The larger XRD value obtained for (a) is due to the cubic structure of this cluster sample and the wide size distribution where larger particles contributed more to the intensity.

Hall method was also applied to these experimental data. The Fourier Transform of these samples are shown in Figure 5.10(a-c). The estimated cluster size for each sample is marked with the grey rectangular region indicating uncertainty intervals around the size estimates: (a) $6.5 \pm 0.5 \mathrm{~nm}$, (b) $5.0 \pm 0.5 \mathrm{~nm}$ and (c) $3.0 \pm 0.5 \mathrm{~nm}$. These values are fairly comparable to TEM values. The Hall method give similar cluster sizes when compared to TEM analysis. Thus, also XRD patterns can be used to gain, accurate, cluster sizes. However, in the following, we will use the sizes obtained by TEM studies only. 


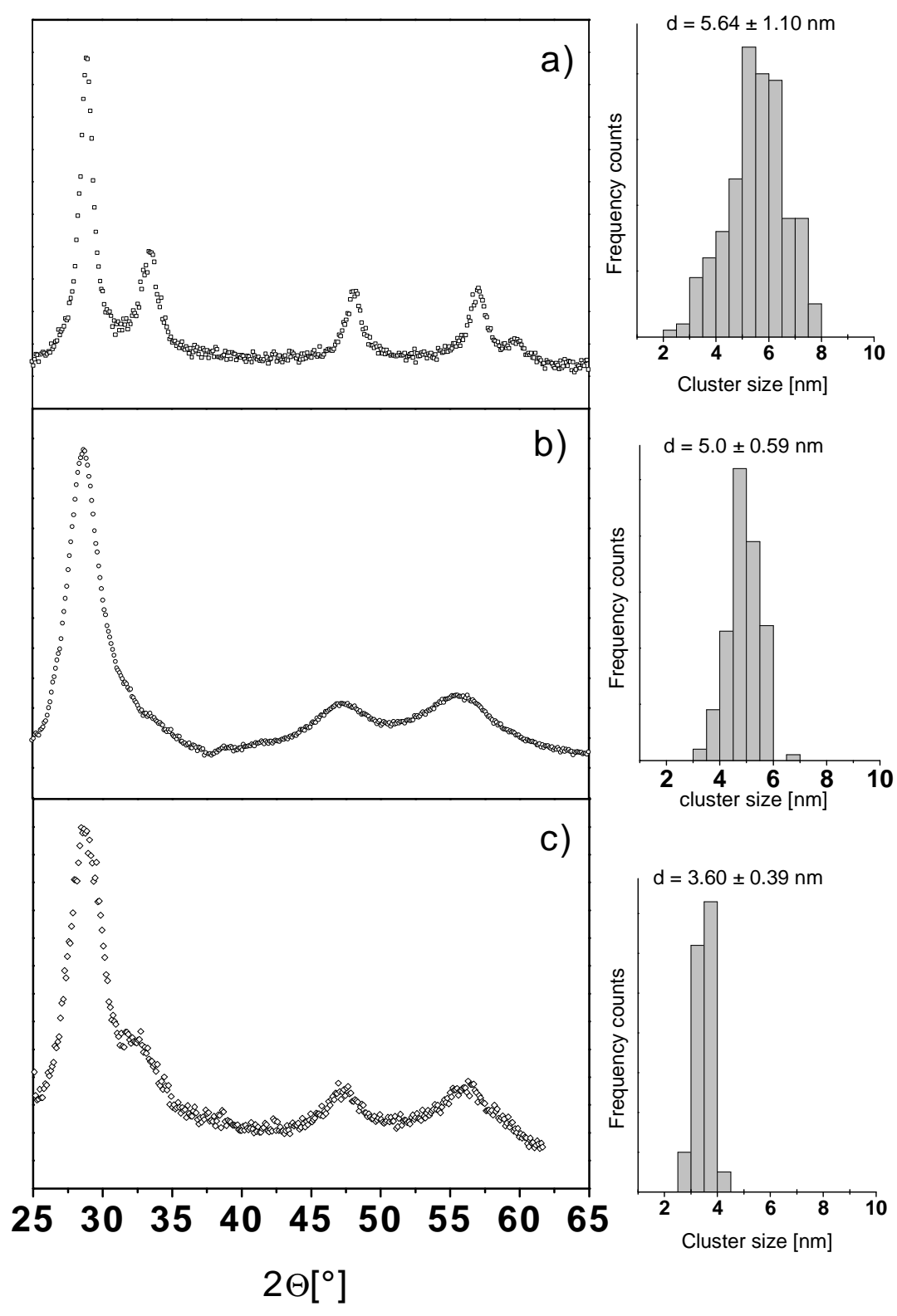

Figure 5. 9. Diffraction patterns of three different samples of electrochemically prepared Pd clusters: sample msE21 (a), sample ms302 (b) and sample ms615 (c). On the side of the diffractogram of each sample the size distribution of the sample obtained by TEM is shown. 

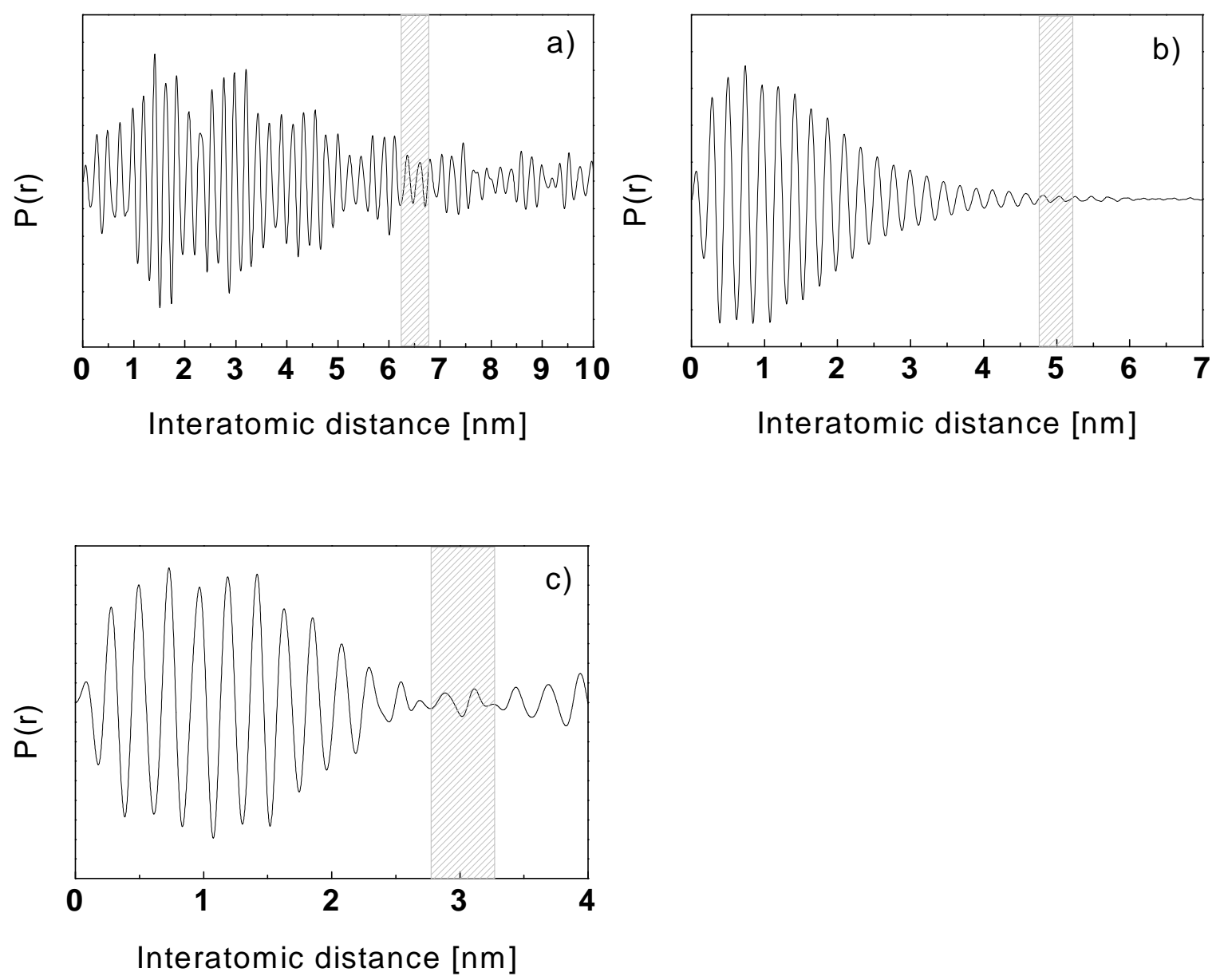

Figure 5. 10. Profiles (a) through (b) show the results of inverting the diffraction patterns of Fig. 5.9. The grey rectangular region indicates the uncertainty interval around the estimated Pd Cluster size. 


\subsection{Cluster Structure}

The structure of the Pd clusters was determined by two independent methods, high resolution electron microscopy (HREM) and X-ray diffraction (XRD). Although HREM is one of the most useful technique to determine the structure, it has the disadvantage of illuminating the sample with a high energy source, in our case $200 \mathrm{kV}$. In some cases it can be as high as 400 $\mathrm{kV}$ this could lead to structural fluctuations of the nanometer-sized cluster and a mixture of structures could be obtained [12]. On the other hand, the XRD is another possible technique which is also powerful in determining the structure.

In this work, comparing the XRD pattern of Pd clusters with XRD patterns obtained from MD-simulations [83] for model Pd clusters in two different lattice structures allows us to determine the structure of the Pd clusters. Jisrawi [83] calculated diffraction pattern of model clusters up to 2057 atoms (about $3.7 \mathrm{~nm}$ ). HREM analysis will be also used to confirm the structure.

Figure 5.7 shows XRD patterns of $3.7 \mathrm{~nm}$ Pd model clusters (a) with cubic lattice structure, and (b) with icosahedral structure. There are important and significant differences between the two patterns. The cubic cluster, Fig.5.7(a) (solid line), has a diffraction patterns similar to that of bulk Pd, whereas the icosahedral cluster has a different diffraction pattern. These features are: First, the intensity of the diffraction peaks (near bulk (200) and (222)) have a smaller amplitude in comparison to the near bulk (111) peak. Second the separation between the peak near (111) and the peak near (220) is less in comparison to that of the bulk. Third, an increased intensity in the diffractogram of the icosahedral cluster between 51 and 56 degrees is also observed.

In the previous section we have shown the XRD patterns of three different experimental cluster samples ((a) $5.6 \mathrm{~nm}$, (b) $5.0 \mathrm{~nm}$ and (c) $3.6 \mathrm{~nm}$ (determined by TEM)) shown in Figure 5.9 (a-c), respectively. In Figure 5.9 (b) and (c), there is a considerable intensity decrease in some diffraction peaks (near bulk (200) and (222)) in comparison with that of the near bulk (111) diffraction pattern. In addition, there is an intensity increase between 51 and 56 degrees in the diffractogram of the $3.6 \mathrm{~nm}$ and $5.0 \mathrm{~nm}$ clusters which was not found for the $5.6 \mathrm{~nm}$ clusters, see Fig. 5.9(a). Furthermore, the reflection close to the bulk (200) peak position is 
slightly shifted towards the reflection near the bulk (111) peak position. Comparing the experimental XRD patterns with those obtained from MD-simulated clusters, shows that the $5.6 \mathrm{~nm}$ clusters have a cubic lattice structure, while the $3.6 \mathrm{~nm}$ and the $5.0 \mathrm{~nm}$ clusters have predominantly icosahedral lattice structure. This can explain the smaller size value obtained when estimating the cluster size of the last two samples using the Scherrer formula as we have seen in the previous section.

The structure of clusters was also determined by analysing the HREM images of the clusters. Fourier transfer analysis (FFT) of the $5.6 \mathrm{~nm}$ cluster sample shows that most of particles $(92 \%)$ have cubic lattice structure, $4 \%$ are icosahedral and others are multi-twinned. Figure 5.11 shows three HREM images of three single clusters of this sample and corresponding FFT analysis. Fig. 5.11(A) shows a cubic particle and it corresponding FFT where in the HREM image the (111) planes are clearly seen as columns of atoms appear as black dots. In corresponding FFT of this particle two dots are visible indicating that the particle is in the $<111>$ orientation. In the HREM images cubic lattices always show lattice fringes (straight lines).

Figure 5.12, shows the HREM and corresponding FFT images of the $5.0 \mathrm{~nm}$ cluster, most of particles (96\%) have icosahedral structure as can be seen in Fig.5.12(a). In Fig. 5.12(a) although there is some background noise one can see 10 spots in the FFT corresponding to five fold symmetry. Very small number are cubic others are single-twined particles, Fig.5.12 (b) \&(c). Generally, icosahedral clusters do not lay in the desired five fold symmetry direction and one has to determine the structure only from the HREM image. In this case, no straight lattice fringes are visible but an amorphous-like pattern. In some cases, other pattern, resulting from the two-or three- fold symmetry direction are visible. However, even in these cases no straight lattice fringes but curved lines are visible.

Figure 5.13, shows the HREM of $3.6 \mathrm{~nm}$ (TEM) Pd cluster sample. Fourier transfer analysis (FFT) of HREM images shows that most of the particles (94\%) have an icosahedral lattice structure. In Fig 5.13(a), although there is some background noise, one can see 10 spot in the FFT corresponding to the five fold symmetry. Few particles are cubic (2\%) and some are multi-twin particles (4\%) (Fig. 5.13 (b)\&(c)). 

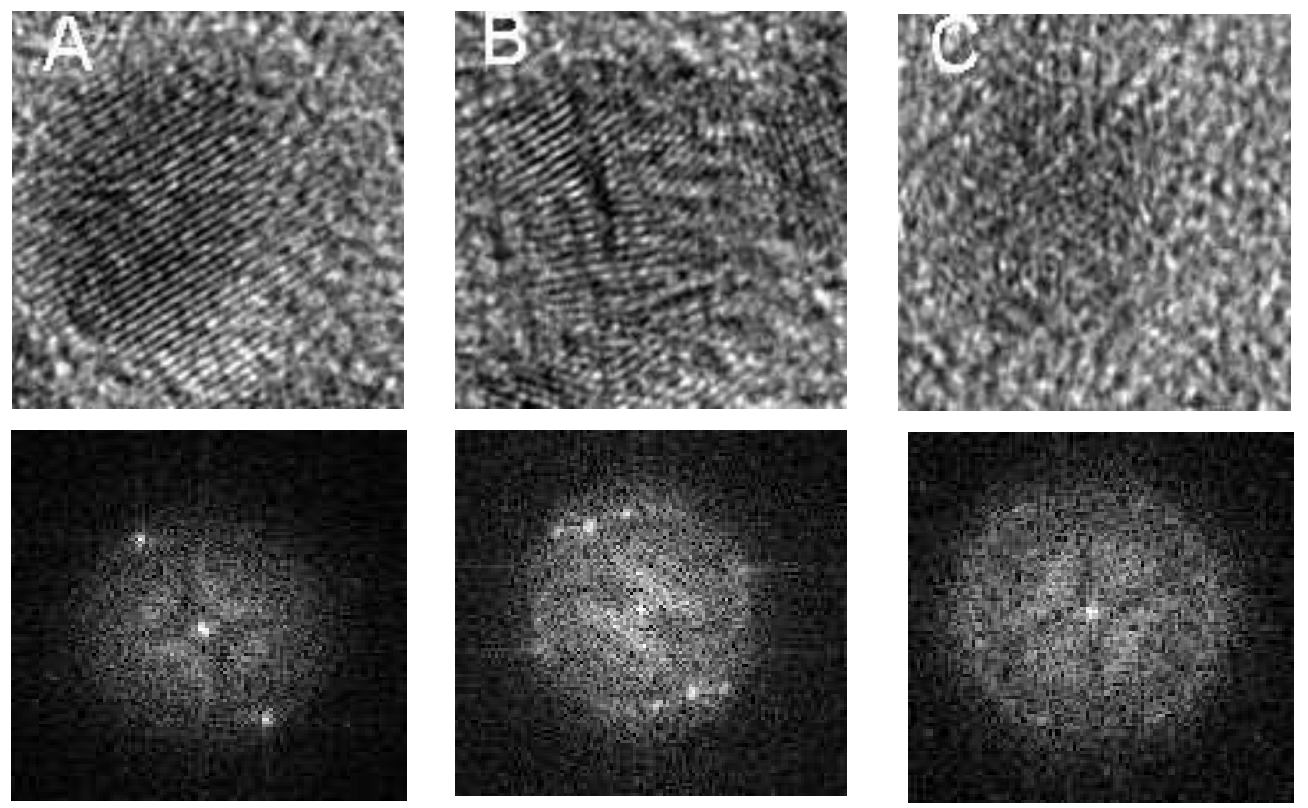

Figure 5. 11. HREM images and diffraction images of the $5.6 \mathrm{~nm}$ cluster: $92 \%$ of the clusters have cubic structure (A). The rest are multi-twinned $(B)$ or icosahedral $(C)$.
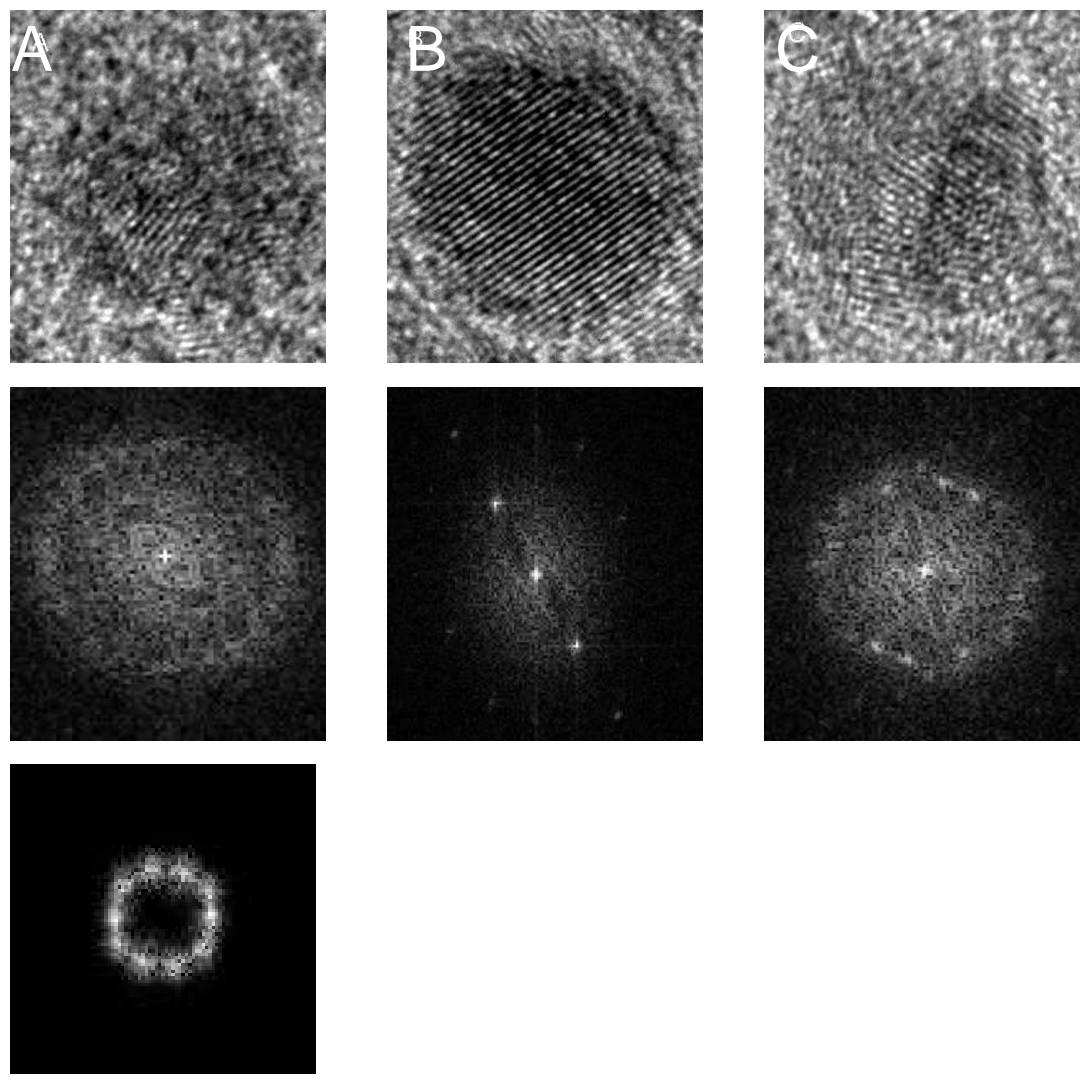

Figure 5. 12. HREM of and FFT images of the $5.0 \mathrm{~nm}$ Pd cluster: $96 \%$ of the particles have icosahedral structure (A), The rest is simple cubic (B) or multi-twinned $(C)$. Fig.5.12(A) bottom shows the FFT images after removing the background. 

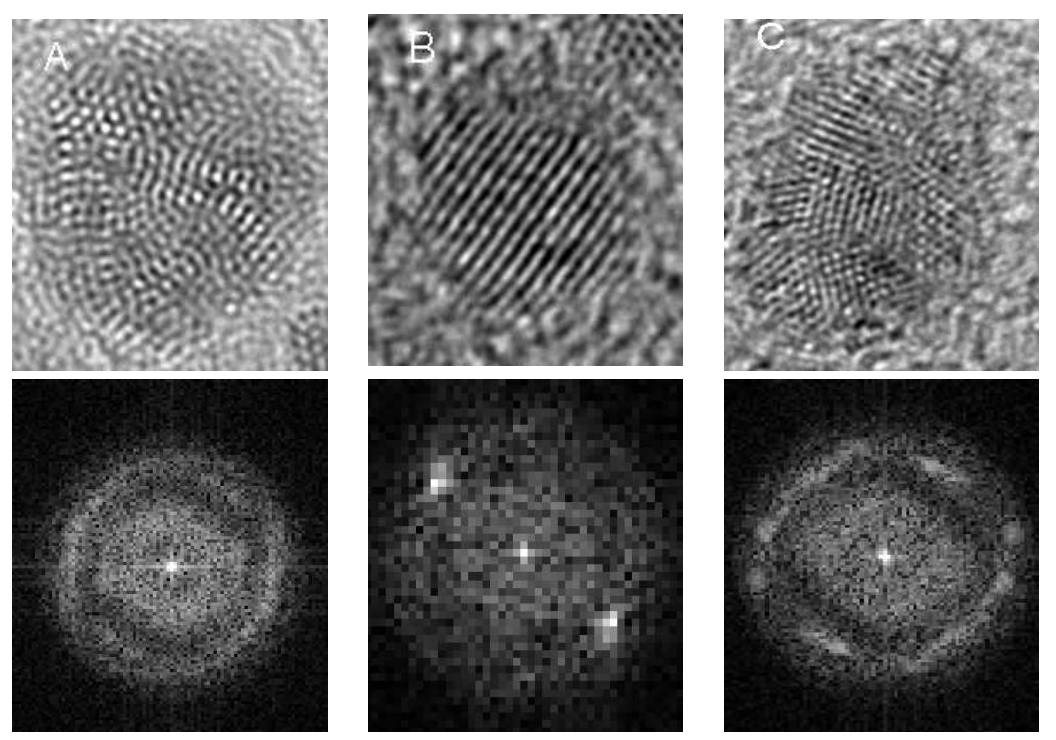

Figure 5. 13. HREM of the $3.6 \mathrm{~nm}$ Pd clusters: $94 \%$ of the particles have icosahedral structure (A). The rest is cubic $(B)$ or multi-twinned $(C)$. 


\section{Results: Pd-H Clusters}

In this chapter experimental results on the thermodynamic and kinetic aspects of the interaction of hydrogen with Pd clusters will be presented. The hydrogenation of the Pd cluster samples, with different sizes, was performed from the gas phase. The palladiumhydrogen Pd-H clusters were studied by gravimetric methods and in-situ X-ray diffraction (XRD) measurements. First gravimetric measurement results, from which the pressureconcentration isotherms $p-x$ - $T$ for different cluster sizes were obtained will be shown. This will be followed by lattice expansion and phase transition results obtained from in-situ XRD measurements during hydrogen loading-unloading. All XRD measurements were performed on the beam line B2 at the Hamburg synchrotron laboratory HASYLAB at DESY. The high intensity of a synchrotron beam enables us to study the hydrogenation behaviour of the $\mathrm{Pd}$ clusters in-situ and has enormously reduced the time needed for the acquisition of the data ${ }^{8}$. The lattice expansion is monitored by the shift in the peaks positions at different hydrogen pressure. The hydride formation is followed by appearance of new set of peaks or by additional peak broadening and changes in the peaks intensities. The in-situ XRD measurements enable us to view the sample lattice structure directly during hydrogen loading. The Pd cluster samples used in the gravimetric and the in-situ XRD measurements were either the same or have comparable cluster sizes.

8 For example the time needed for a measurement in the $2 \theta$ range $24^{\circ}$ to $65^{\circ}$ is about $30 \mathrm{~min}$. If the same measurement where to be done with another x-ray source, e.g. Co $\mathrm{K} \alpha$, the time needed would be more than 48 hours. 


\subsection{Pressure-concentration Isotherms}

In this section experimental absorption-desorption isotherms, obtained by gravimetric measurements of different cluster sizes will be presented. Absorption-desorption measurements were performed after a pretreatment step which is necessary to reduce any oxide layer. Details about the pretreatment step and absorption-desorption procedure were given in chapter 3 .

Table 6.1 summarises the samples used in these measurements. For comparison Pd-powder (99.9\% Pd with particle size of about $400 \mu \mathrm{m}$ ) was also used. As have been shown in section 5.4 the cluster size value obtained by TEM is more accurate than that estimated from the XRD data by using the Scherrer formula. Therefore, we will refer to the cluster size by the value obtained by TEM.

Table 6. 1. Experimental data for the used Pd cluster samples: showing the cluster size and \%Pd (wt\%). The cluster size is obtained by TEM and estimated from XRD data using the Scherrer formula.

\begin{tabular}{c|ccc}
\hline Sample No. & \multicolumn{2}{|c}{ Size [nm] } & $\% P d$ \\
\cline { 2 - 3 } & TEM & XRD & \\
Ms706 & $5.2 \pm 0.6$ & 3.7 & 33.92 \\
Ms718 & $4.8 \pm 0.8$ & 3.4 & 32.30 \\
msE18 & $3.6 \pm 0.6$ & 2.1 & 51.06 \\
msE13 & $3.0 \pm 0.5$ & 2.0 & 60.05 \\
\hline
\end{tabular}

Figure 6.1 shows the experimental profile of two different cluster samples. It is clearly visible that the surfactant stabilised Pd clusters absorbed hydrogen in a fast way. This is in accordance with the in-situ XRD measurements where it was noticed that the kinetic of hydrogen absorption is fast as will be shown in section (6.2). An equilibrium pressure was obtained within 1hour in most cases, in the two-phase field longer time is required. Although the hydrogen absorption kinetic is improved in comparison to polymer stabilised clusters the 
hydrogen desorption need longer time. These results are in accordance with previous volumetric measurements performed by our group.

From the gravimetric measurements equilibrium data were taken to construct the pressureconcentration isotherms. Figure 6.2 shows the absorption-isotherms for the four different cluster samples and absorption isotherm of the Pd-powder. The absorption isotherm of the $400 \mu \mathrm{m}$ Pd-powder shows three well-distinguished parts and shows to be comparable to the $p$ $x$ - $T$ isotherms obtained by hydrogen absorption in bulk Pd shown in Fig.1.1 in chapter 1.

The isotherms for each cluster sample were obtained from three different absorption cycles and turned out to be reproducible at low hydrogen pressure. However, at higher pressure the isotherms reveals a decrease in the hydrogen concentration with repeating the absorption cycle. In all clusters isotherms the amount of total absorbed hydrogen in the first absorption cycle is larger than that in the second cycle which again is larger than that in the third absorption cycle.

The cluster absorption-isotherms show the following characteristics: First, an enhanced hydrogen solubility at low hydrogen pressure is observed as compared to Pd-powder. Second, there are three distinguished parts in the isotherms of the $5.2 \mathrm{~nm}$, the $4.8 \mathrm{~nm}$ and the $3.6 \mathrm{~nm}$ cluster samples. These parts are marked with the dotted lines see Fig. 6.2(b-d). In the second part the hydrogen concentration increases largely at nearly constant hydrogen pressure. No such region was found in the $p-x-T$ isotherm of the smallest cluster $(3.0 \mathrm{~nm})$ where only two distinguished parts (or parts with different slope) can be seen, as shown in Fig. 6.2(e). Third, the "plateau" in the two-phase region has a slope instead of being flat as expected for bulk $\mathrm{Pd}$. Moreover cluster samples have smaller plateau-pressures than the Pd-powder. Fourth, the two-phase region found in the isotherms of the $(5.2 \mathrm{~nm}, 4.8 \mathrm{~nm}$ and $3.6 \mathrm{~nm})$ cluster samples is narrowed as compared the two-phase region found in the Pd-powder, and it becomes narrower with decreasing the cluster size.

From the $p-x-T$ isotherms the questions are arising: if the slopes plateau region is really marking a phase transition and if two phases coexist within the sloped plateau region of the clusters, which phases appear and, in case of the smallest clusters, if there is a phase transition. These questions will be under the scope of investigation in the in-situ XRD measurements in the next section. 

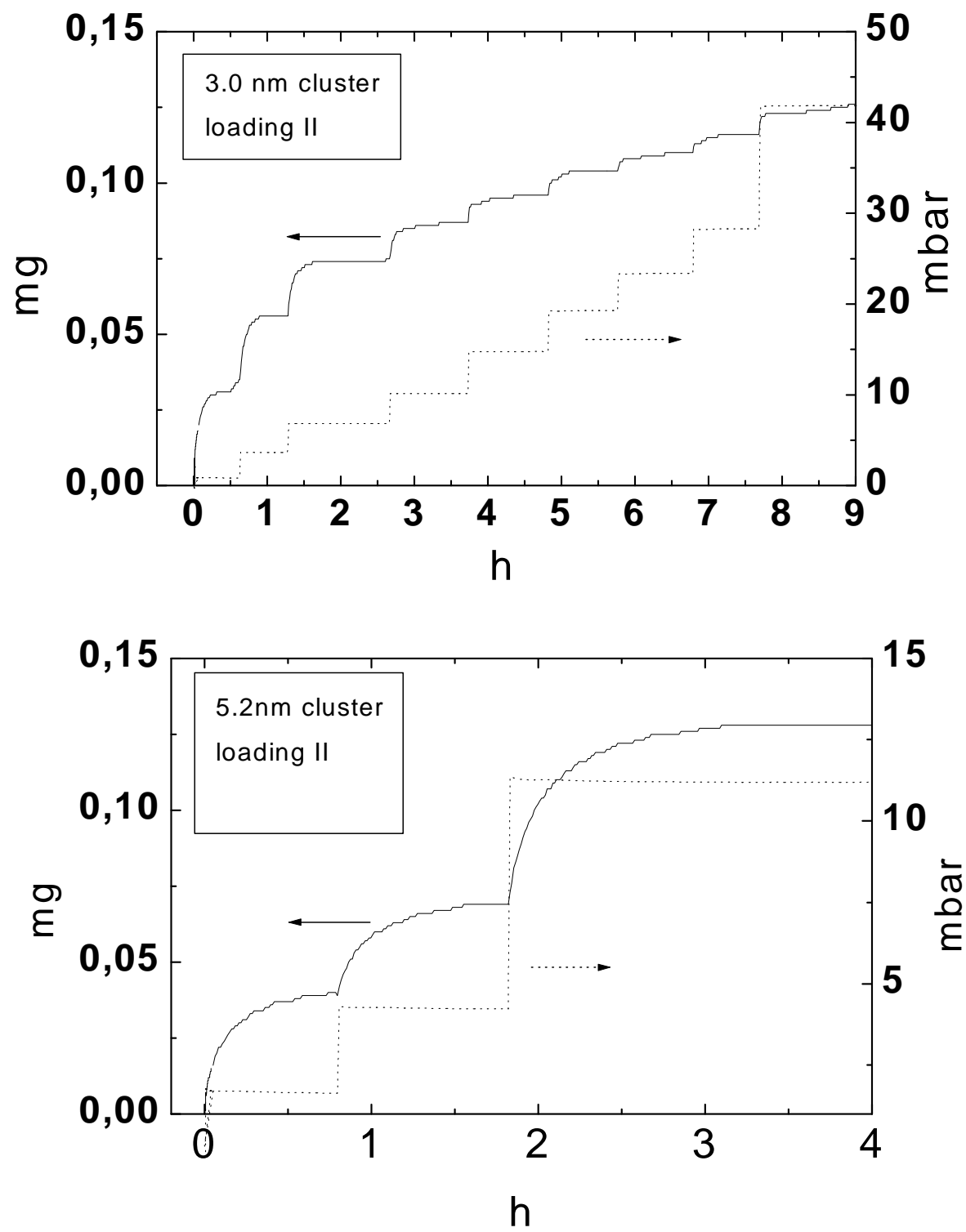

Figure 6. 1 Reaction profile of the Gravimetric measurements for two cluster samples: $3.0 \mathrm{~nm}$ (upper graph) and $5.2 \mathrm{~nm}$ (down graph). The left y-axes shows the amount of hydrogen absorbed (solid lines) at different hydrogen pressure loading steps (dotted line right y-axis). 
(a) Pd powder

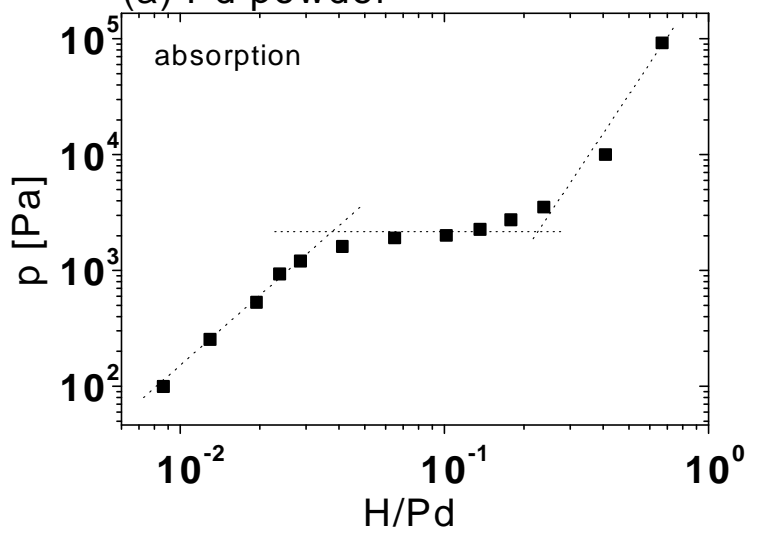

(c) $4.8 \mathrm{~nm}$ cluster

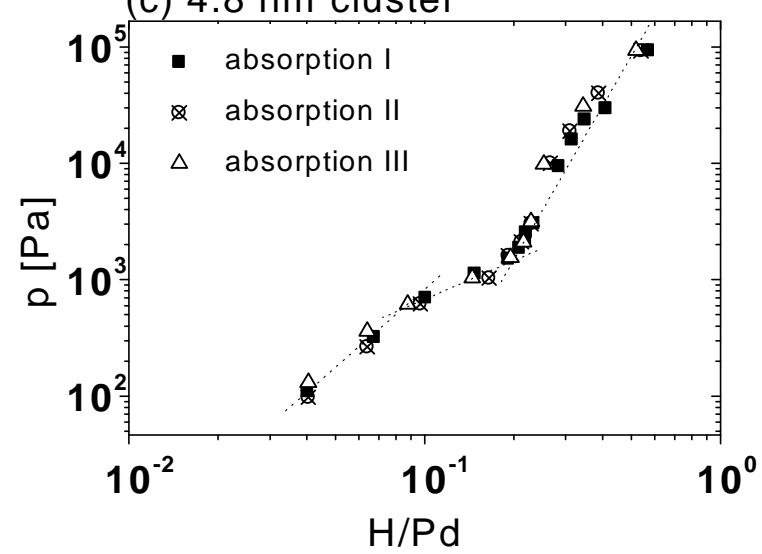

(e) $3.0 \mathrm{~nm}$ cluster

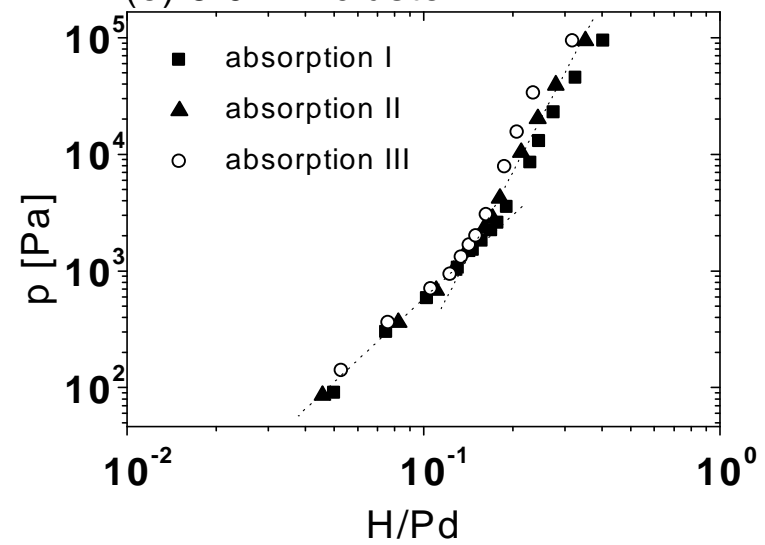

(b) $5.2 \mathrm{~nm}$ cluster

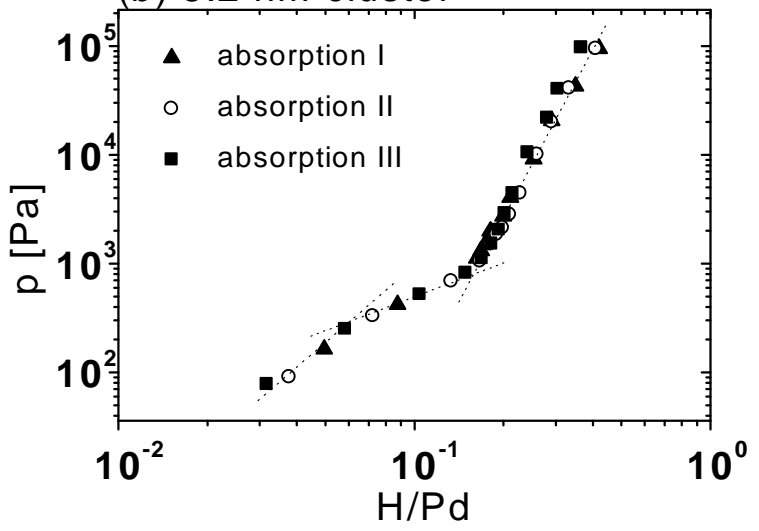

(d) $3.6 \mathrm{~nm}$ cluster

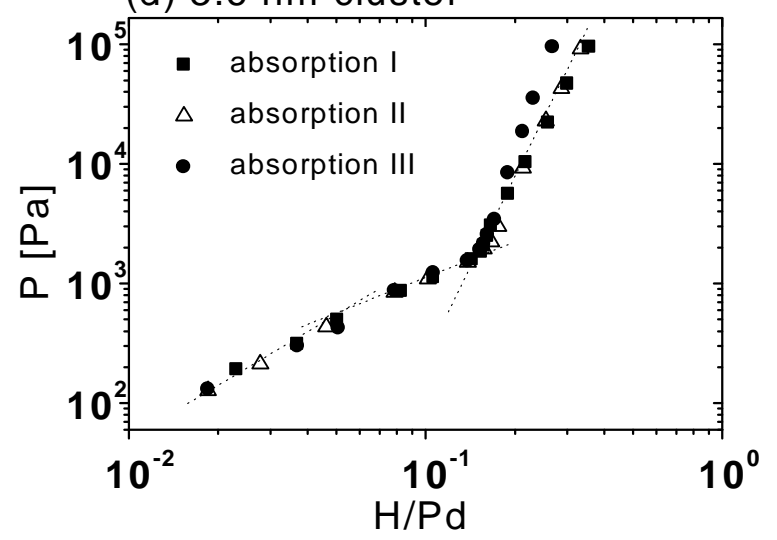

Figure 6. 2. Absorption isotherms for different samples: $400 \mu \mathrm{m}$ Pd powder (a), $5.2 \mathrm{~nm}$ cluster sample (b), $4.8 \mathrm{~nm}$ cluster sample (c),3.6 $\mathrm{nm}$ cluster sample (d) and $3.0 \mathrm{~nm}$ cluster sample (e). All isotherms show an increased hydrogen solubility at low hydrogen pressure. In isotherms (a) through (d) three distinguishable parts can be seen, isotherm (e) shows only two distinguished parts. 


\subsection{Lattice changes during loading-unloading with}

\section{hydrogen}

In-situ X-ray diffraction measurements for clusters with different sizes $(3.0 \mathrm{~nm}, 3.6 \mathrm{~nm}, 4.8$ $\mathrm{nm}, 5.6 \mathrm{~nm}, 6.0 \mathrm{~nm}$ ) were performed during hydrogen loading-unloading at different hydrogen pressures. In order to remove any oxide all cluster samples were pretreated with hydrogen before starting a loading-unloading cycle. One loading-unloading cycle consists of about 30 different pressure steps. The measurements were taken, in most cases, in the $2 \theta$ range from $24^{\circ}$ to $65^{\circ}$. The results of these measurements will be shown in the following sections. Part of these results is already published in $[84,85]$.

Table 6.2 summarises the samples used in these measurements. The cluster size and lattice structure of these samples were already discussed in sections (5.3) and (5.4). The cluster samples used in these study where either the same (samples: ms718 and msE13) or have comparable sizes to those used in the gravimetric study.

Table 6. 2. Diameter of the used Pd cluster samples The cluster size is obtained by TEM and estimated from XRD data using the Scherrer formula

\begin{tabular}{c|cc}
\hline Sample No. & size $[\mathrm{nm}]($ TEM) & size $[\mathrm{nm}](\mathrm{xrd})$ \\
\hline $\mathrm{ms} 717$ & $6.0 \pm 1.3$ & 8.0 \\
$\mathrm{msE} 21$ & $5.6 \pm 1.1$ & 6.0 \\
$\mathrm{~ms} 302$ & $5.0 \pm 0.6$ & 3.5 \\
$\mathrm{~ms} 718$ & $4.8 \pm 0.8$ & 3.4 \\
$\mathrm{~ms} 615$ & $3.6 \pm 0.4$ & 2.2 \\
$\mathrm{msE} 13$ & $3.0 \pm 0.5$ & 2.0 \\
\hline
\end{tabular}




\subsubsection{Hydrogenation of $6.0 \mathrm{~nm}$ clusters}

The XRD patterns of the as-prepared sample indicates a typical cubic structure (Figure 6.3). The clusters exhibit diffraction peaks at the same position as bulk Pd, whereas the peaks are broader. In addition, the relative intensities of the peaks are the same as those expected from bulk Pd.

During hydrogen loading, a shift to smaller $2 \theta$ values is observed in the diffraction patterns, indicating a lattice expansion. Figure 6.3 shows diffraction patterns of the $6.0 \mathrm{~nm}$ cluster sample at four different pressures in comparison with the as-prepared sample, monitoring the lattice expansion by a shift of the peak positions. However this shift is very small at low hydrogen pressure $\left(1.0 \times 10^{3} \mathrm{~Pa}\right)$ and is more visible at higher pressure $\left(1.1 \times 10^{4} \mathrm{~Pa}\right)$. The cluster maintain its original cubic lattice structure during the hydrogen absorption.

In this cluster sample, a transition is observed in the pressure range of $1.5 \cdot 10^{3}-2.5 \cdot 10^{3} \mathrm{~Pa}$ [85]. Figure 6.4 shows the XRD patterns of this sample at four different hydrogen pressure monitoring the phase transition in detail between $25^{\circ}$ and $45^{\circ}$. Since these clusters have a cubic lattice structure and, since, the observed transition occurs at a pressure typical for bulk palladium, this transition is interpreted as the $\left(\alpha-\alpha^{\prime}\right)$ phase transition known from bulk Pd. At $1.8 \cdot 10^{3} \mathrm{~Pa}$ another set of Bragg reflections start to appear (belonging to the $\alpha$-cubic structure also) indicating the formation of the $\left(\alpha^{\prime}-\mathrm{Pd}-\mathrm{H}\right)$ phase. At higher pressure, the whole cluster sample transfers into the $\left(\alpha^{\prime}-\mathrm{Pd}-\mathrm{H}\right)$ phase and the $(\alpha-\mathrm{Pd}-\mathrm{H})$ phase peaks completely vanish. Figure 6.5 shows that during hydrogen unloading a shift to higher $2 \theta$ values is observed. This indicates that as hydrogen is desorbed from the sample the lattice relaxes. Decreasing the hydrogen pressure the intensity of the $\left(\alpha^{\prime}-\mathrm{Pd}-\mathrm{H}\right)$ phase peaks decreases and at lower pressure it vanishes completely and the sample is in the $(\alpha-\mathrm{Pd}-\mathrm{H})$ phase. After unloading the diffraction patterns of the sample are at the exact positions of the as-prepared one. This indicates that hydrogen can absorb and desorp reversibly in this cluster sample.

During unloading the $\left(\alpha^{\prime}-\alpha\right)$ phase transition can also be observed. The $(\alpha-\mathrm{Pd}-\mathrm{H})$ phase peaks start to grow at $1.0 \times 10^{3} \mathrm{~Pa}$ hydrogen pressure, Figure 6.6. At $8.5 \times 10^{2} \mathrm{~Pa}$ the phase separation is visible and a new sets of Bragg reflections is observed corresponding to the $(\alpha-\mathrm{Pd}-\mathrm{H})$ phase. 


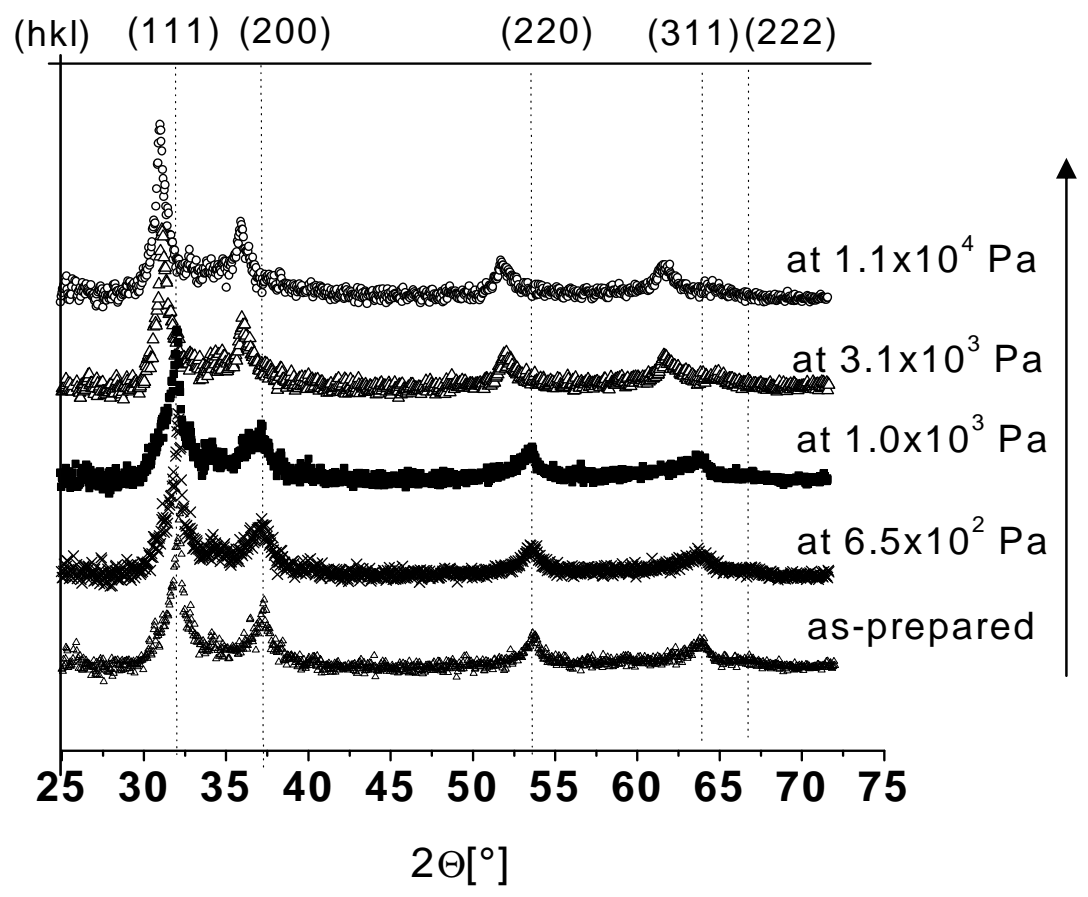

Figure 6. 3. Diffraction patterns of the $6.0 \mathrm{~nm}$ cluster sample monitoring the lattice expansion by the shift of the peak positions at different hydrogen pressure. The peak positions of the as-prepared state is marked with the vertical dotted lines. $\lambda=1.24438 \AA$.

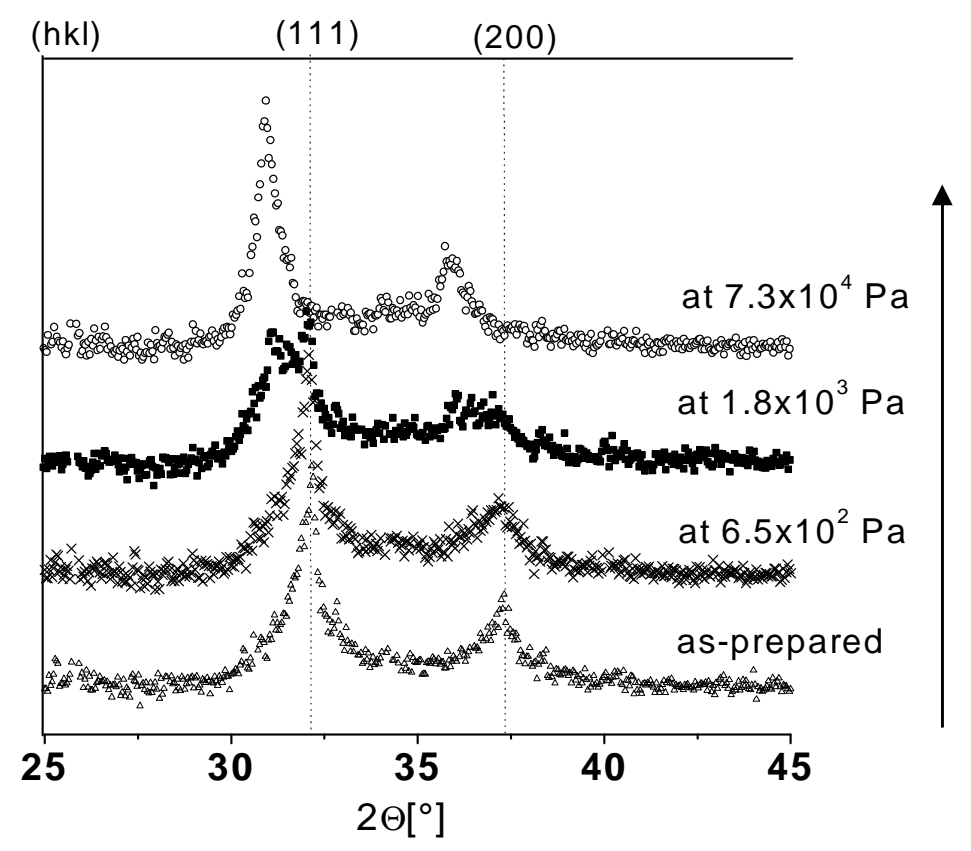

Figure 6. 4. Diffraction patterns of the $6.0 \mathrm{~nm}$ cluster sample at four different hydrogen pressures monitoring the phase transition: Diffraction pattern of as-prepared clusters (up-triangles), at $6.5 \times 10^{2}$ $\mathrm{Pa}$ (crossed line), at $1.8 \times 10^{3} \mathrm{~Pa}$ (squares) and at $7.3 \times 10^{4} \mathrm{~Pa}$ (circles).The $\alpha^{\prime}-\mathrm{Pd}$-H phase is visible at $1.8 \times 10^{3} \mathrm{~Pa}$. At the largest pressure the whole sample is in the $\alpha^{\prime}-P d-H$ phase. $\lambda=1.24438 \AA$. 


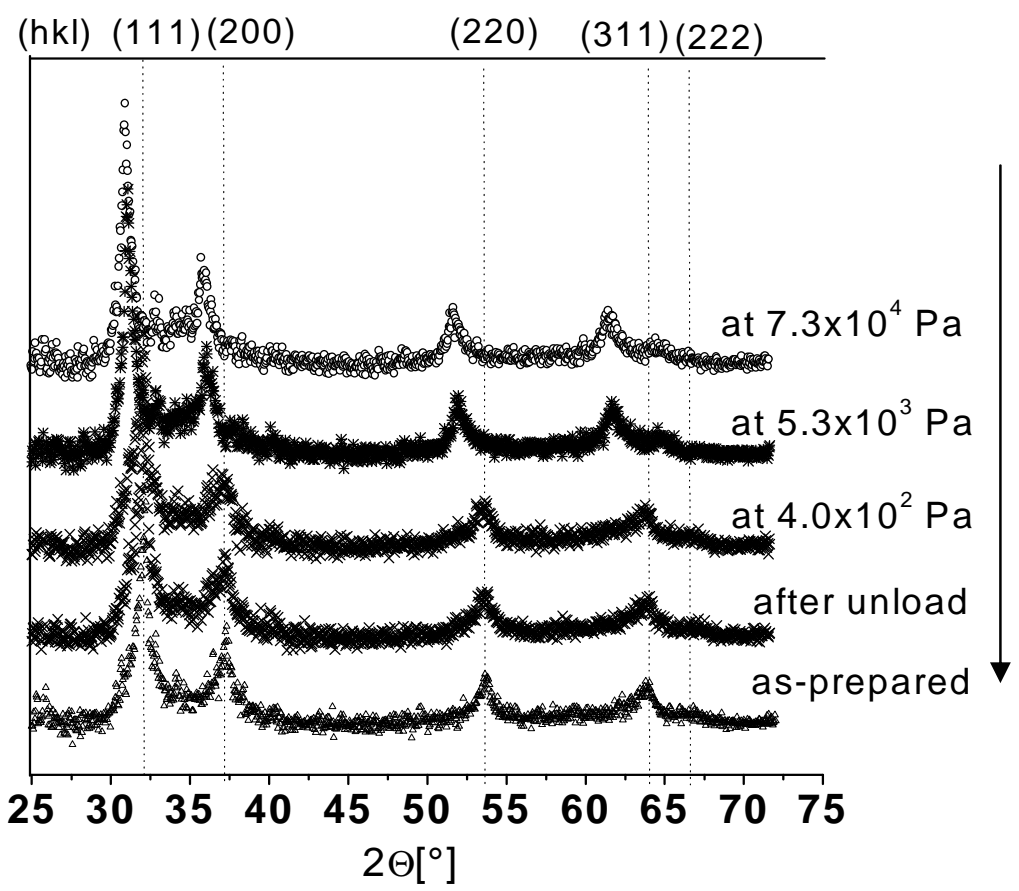

Figure 6. 5. Diffraction patterns of the $6.0 \mathrm{~nm}$ sample monitoring the lattice relaxation during hydrogen unloading by the peak positions. As the hydrogen pressure decreases the lattice relaxes and the peaks shift to higher $2 \theta$-values. After unloading cycle the diffraction patterns are in the same position as that of the as-prepared sample. The vertical dotted lines to marked the peak positions of the as-prepared state. $\lambda=1.24438 \AA$.

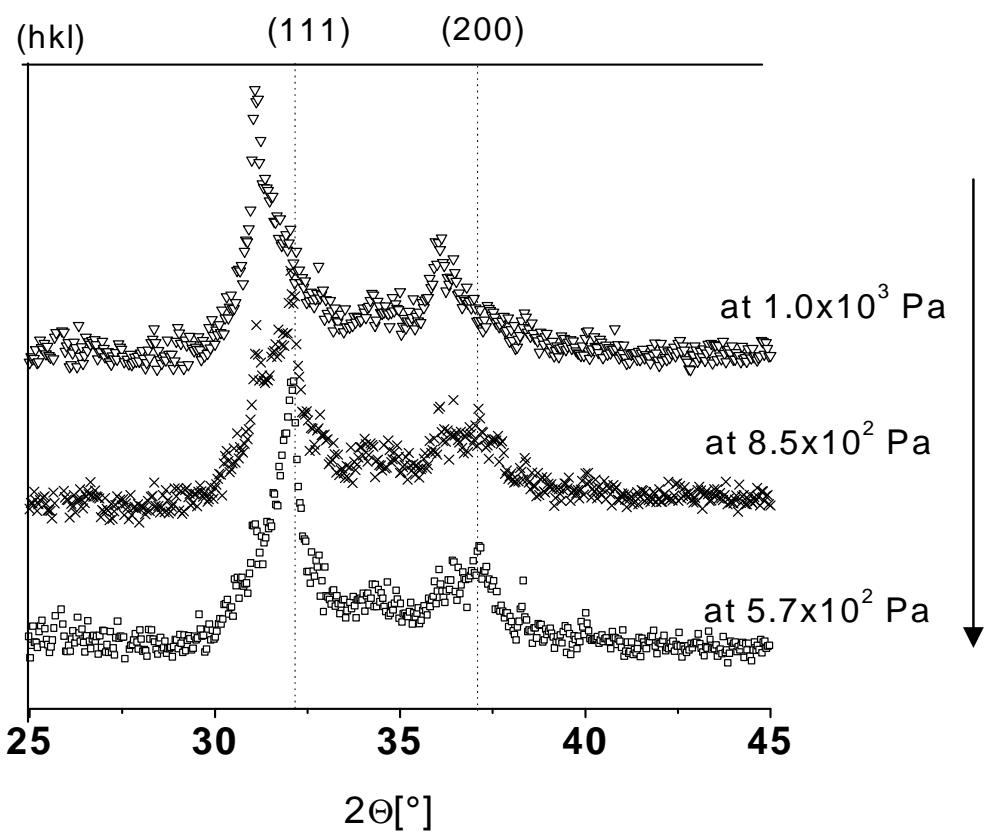

Figure 6. 6. Diffraction patterns of the $6.0 \mathrm{~nm}$ sample during hydrogen unloading. $(\alpha-P d-H)$ phase start to grow at $1.0 \times 10^{3} \mathrm{~Pa}$ and increases with decreasing the pressure. At 5.7 $\times 10^{2} \mathrm{~Pa}$ the whole sample is in the $(\alpha-P d-H)$ phase and the $\left(\alpha^{\prime}-P d-H\right)$ phase vanishes. The vertical dotted lines are to marked the peaks position of the $(\alpha-P d-H)$ phase. $\lambda=1.24438 \AA$. The small peak arise around $34^{\circ}$ is a background peak. 


\subsubsection{Hydrogenation of $5.6 \mathrm{~nm}$ clusters}

Figure 6.7 shows the XRD patterns of the as-prepared sample (solid line), at $8 \times 10^{4} \mathrm{~Pa}$ hydrogen pressure (dotted line) and after subsequent pumping (dashed line). The as-prepared sample exhibited a cubic structure, Fig. 6.7 (solid line). The peaks positions and relative intensities are the same as those expected from bulk. The structure of this cluster sample was also discussed in section (5.4).

Hydrogen absorption causes a lattice expansion all the diffraction peaks are shifted towards lower $2 \theta$ values, Fig. 6.7 (dotted line). As in the larger sample $(6.0 \mathrm{~nm})$ hydrogen can absorb and desorb reversibly in this sample, after unloading the XRD patterns are at the same positions as those of the as-prepared one, Fig. 6.7 (dashed lines). All the observed features are similar to those observed from the $6.0 \mathrm{~nm}$ clusters sample.

Stepwise loading cycle was performed while in-situ XRD measurements were taken at each hydrogen pressure step. During hydrogen loading an $\left(\alpha-\alpha^{\prime}\right)$ phase transition is observed in the pressure range of $1.8 \times 10^{3}$ to $3.0 \times 10^{3} \mathrm{~Pa}$. Figure 6.8 shows the diffraction patterns of this cluster sample at four hydrogen pressures monitoring the $\left(\alpha-\alpha^{\prime}\right)$ phase transition. At $1.8 \times 10^{3}$ $\mathrm{Pa}$ the $\alpha^{\prime}-\mathrm{Pd}-\mathrm{H}$ peaks are visible and grow with increasing hydrogen pressure. At the same time the maximum intensity of $\alpha-\mathrm{Pd}-\mathrm{H}$ peaks decreases and vanishes completely at higher pressure, see Fig. 6.8.

During the unloading cycle an $\left(\alpha^{\prime}-\alpha\right)$ phase transition was also observed, as shown in Figure 6.9. The $\alpha-\mathrm{Pd}-\mathrm{H}$ phase starts to grow at $1.9 \times 10^{3} \mathrm{~Pa}$ and increases as the hydrogen pressure decreases. At $6.0 \times 10^{2} \mathrm{~Pa}$ the whole sample is in the $\alpha-\mathrm{Pd}-\mathrm{H}$ phase. 


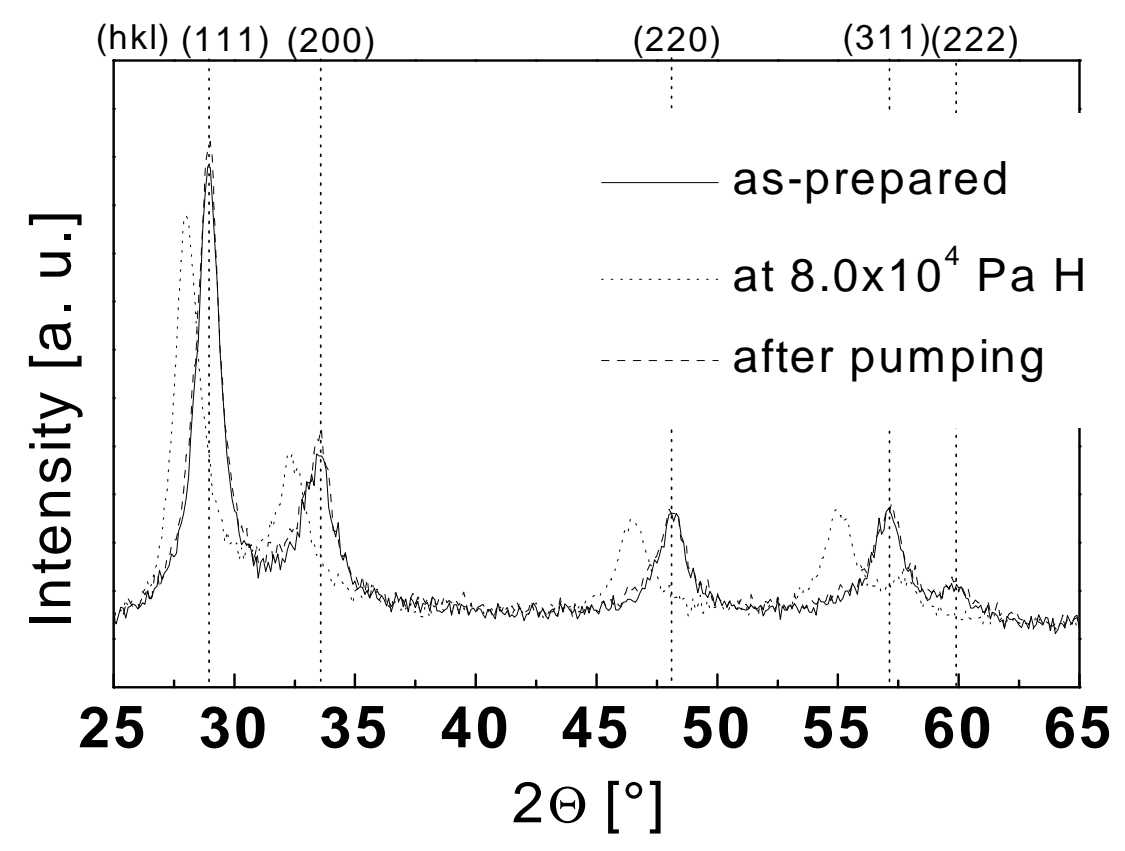

Figure 6. 7. X-ray diffraction patterns of the $5.6 \mathrm{~nm}$ as-prepared sample (solid line), at $8.0 \times 10^{4} \mathrm{~Pa}$ hydrogen pressure (dotted line) and after subsequent pumping (dashed line). $\lambda=1.120599 \AA$.

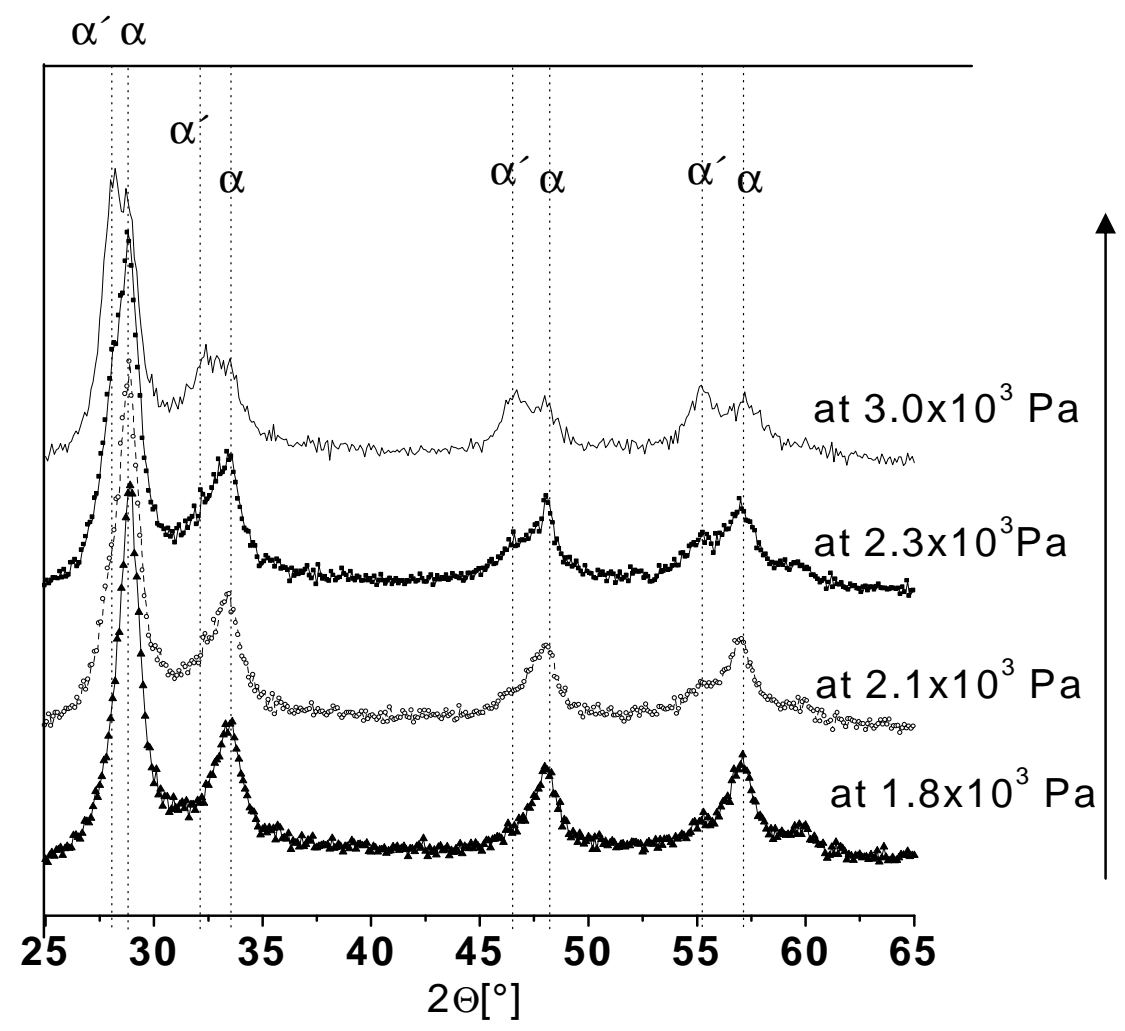

Figure 6. 8. Diffraction patterns of the $5.6 \mathrm{~nm}$ cluster sample during hydrogen loading. $\alpha^{\prime}-P d-H$ phase start to grow at $1.8 \times 10^{3} \mathrm{~Pa}$ and increases with increasing the hydrogen pressure. The vertical dotted lines mark the peaks positions of the $\alpha-P d-H$ and $\alpha^{\prime}-P d-H$ phase of the cluster. $\lambda=1.120599 \AA$. 


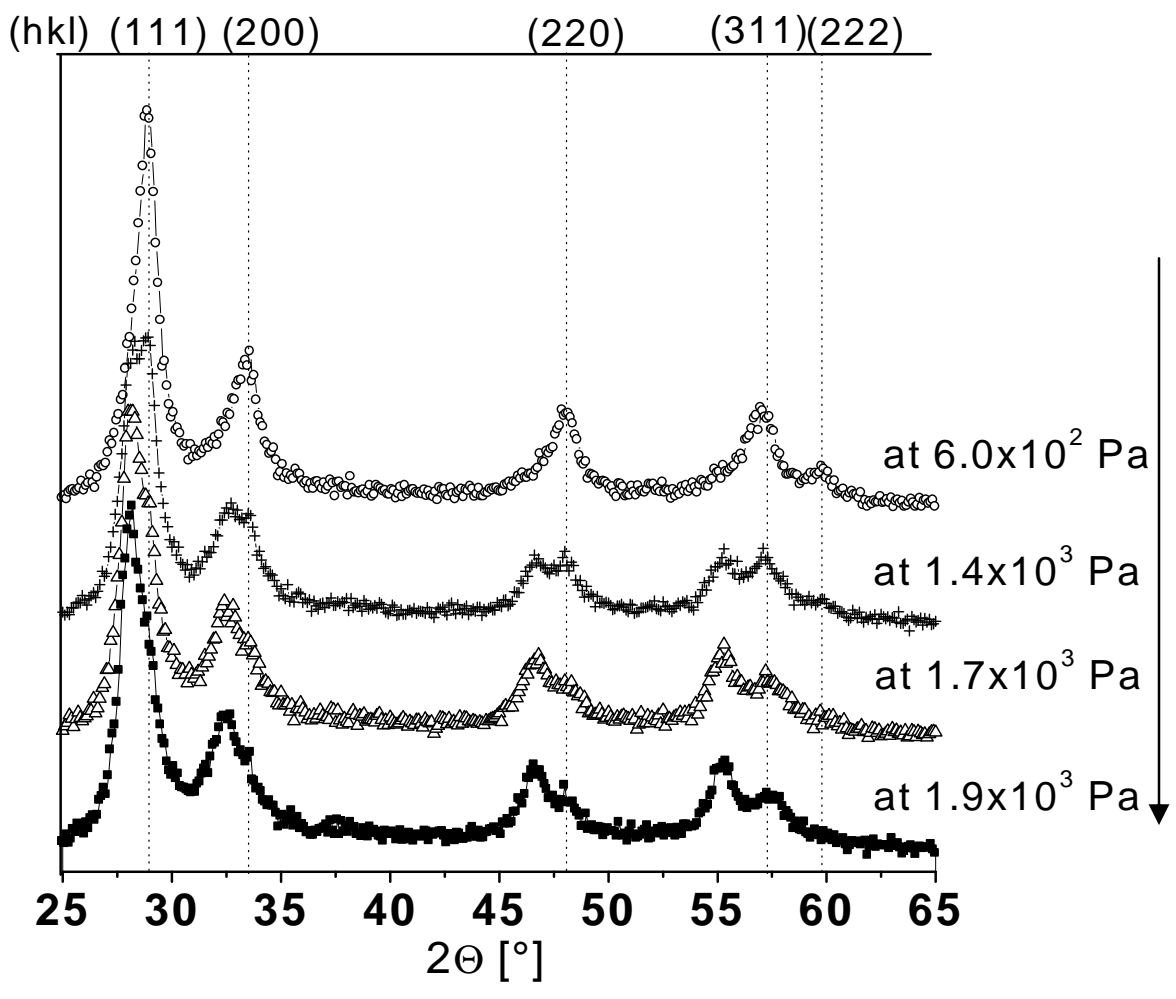

Figure 6. 9. Diffraction patterns of the $5.6 \mathrm{~nm}$ cluster sample during hydrogen unloading. $\alpha-P d-H$ phase start to grows at $1.9 \times 10^{3} \mathrm{~Pa}$ and increases with decreasing the hydrogen pressure. The vertical dotted lines mark the peaks positions of the $\alpha-P d-H$ phase. $\lambda=1.120599 \AA$. 


\subsubsection{Hydrogenation of $5.0 \mathrm{~nm}$ clusters}

Figure 6.10(a) shows a comparison between the XRD patterns of the as-prepared cluster (open-circles), with that of the XRD patterns of the cluster after exposure to $1.0 \times 10^{5} \mathrm{~Pa}$ hydrogen gas for about 12 hours and subsequent unloading (up-triangles lines) shows that: First, there is an increase in the peaks maximum intensity (peaks height) after the absorption of hydrogen. Second, the peaks width becomes narrower as the cluster absorbs hydrogen. Third, there is intensity decrease between $50^{\circ}-55^{\circ}$ after the absorption of hydrogen. Fig.6.10(b) shows additionally that the peaks intensity does not decrease after unloading the sample, as it was observed from smaller sample $(4.8 \mathrm{~nm})$, see section (6.2.4). The XRD patterns of the as-prepared cluster shows a typical icosahedral diffraction pattern: There is a considerable intensity decrease in the diffraction peaks (near bulk (200) and (222)) in comparison to lower angle peak (near bulk (111)).Also, the reflection close to the bulk (200) position are shifted towards the reflection near bulk (111) peak position. The icosahedral structure was ,also, confirmed by HREM analysis, see section (5.4).

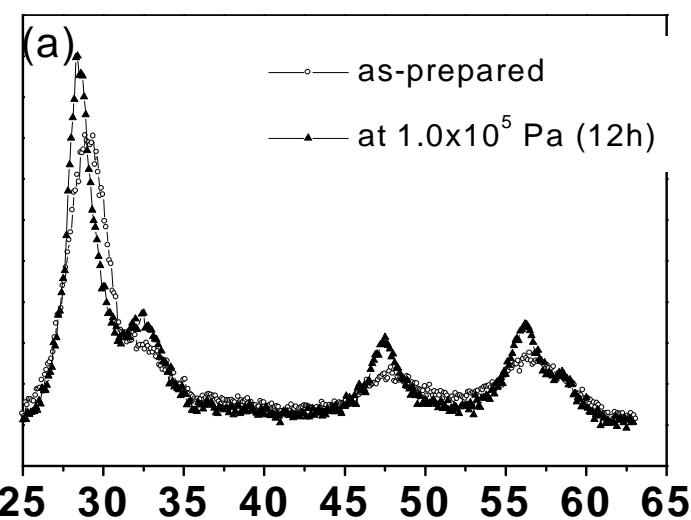

$2 \Theta\left[^{\circ}\right]$

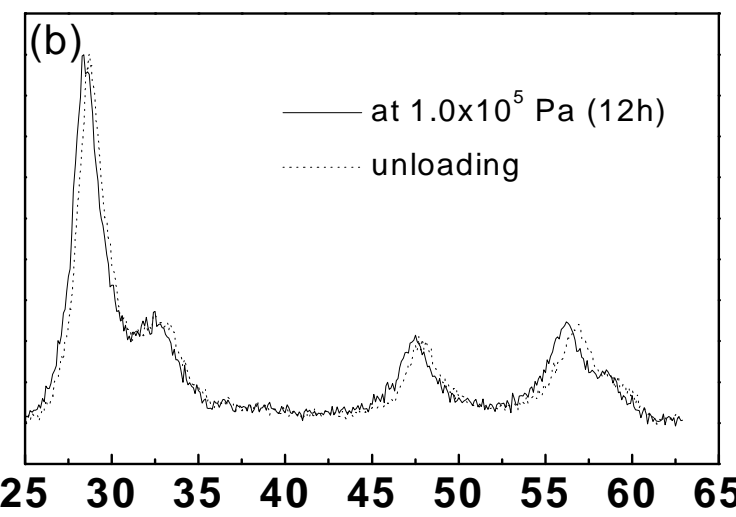

$2 \Theta\left[^{\circ}\right]$

Figure 6. 10(a) Diffraction patterns of as-prepared cluster(triangle) in comparison with that after

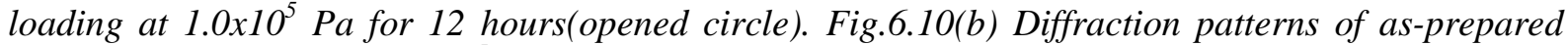
cluster after loading at 1.0x10 $\mathrm{Pa}$ for 12 hours (solid line) in comparison with: that after unloading (dotted line). $\lambda=1.13999 \AA$.

Figure 6.11(a) shows the XRD patterns of the $5.0 \mathrm{~nm}$ cluster at different hydrogen pressures during the first loading cycle. Hydrogen absorption leads to lattice dilatation, shifting the peaks positions to smaller $2 \theta$ values. The lattice expansion increases with increasing the hydrogen pressure. The same behaviour was also observed in a second loading cycle. Figure 
6.11(a) shows the XRD patterns of this sample at three different hydrogen pressures: at $5 \times 10^{2}$ $\mathrm{Pa}$ (dotted line), at $2.1 \times 10^{3} \mathrm{~Pa}$ (dashed line) and at $8.0 \times 10^{4} \mathrm{~Pa}$. A lattice expansion and a slight increase in the peaks intensity is observed as the hydrogen pressure increases. Figure 6.11(b) shows the XRD patterns of this sample during hydrogen unloading. As the hydrogen pressure decreases the peaks position shifts to higher $2 \theta$ values indicating a relaxation in the lattice. Surprisingly, no $\left(\alpha-\alpha^{\prime}\right)$ phase transition was observed. Also there is no change in the peaks intensity during hydrogen absorption.
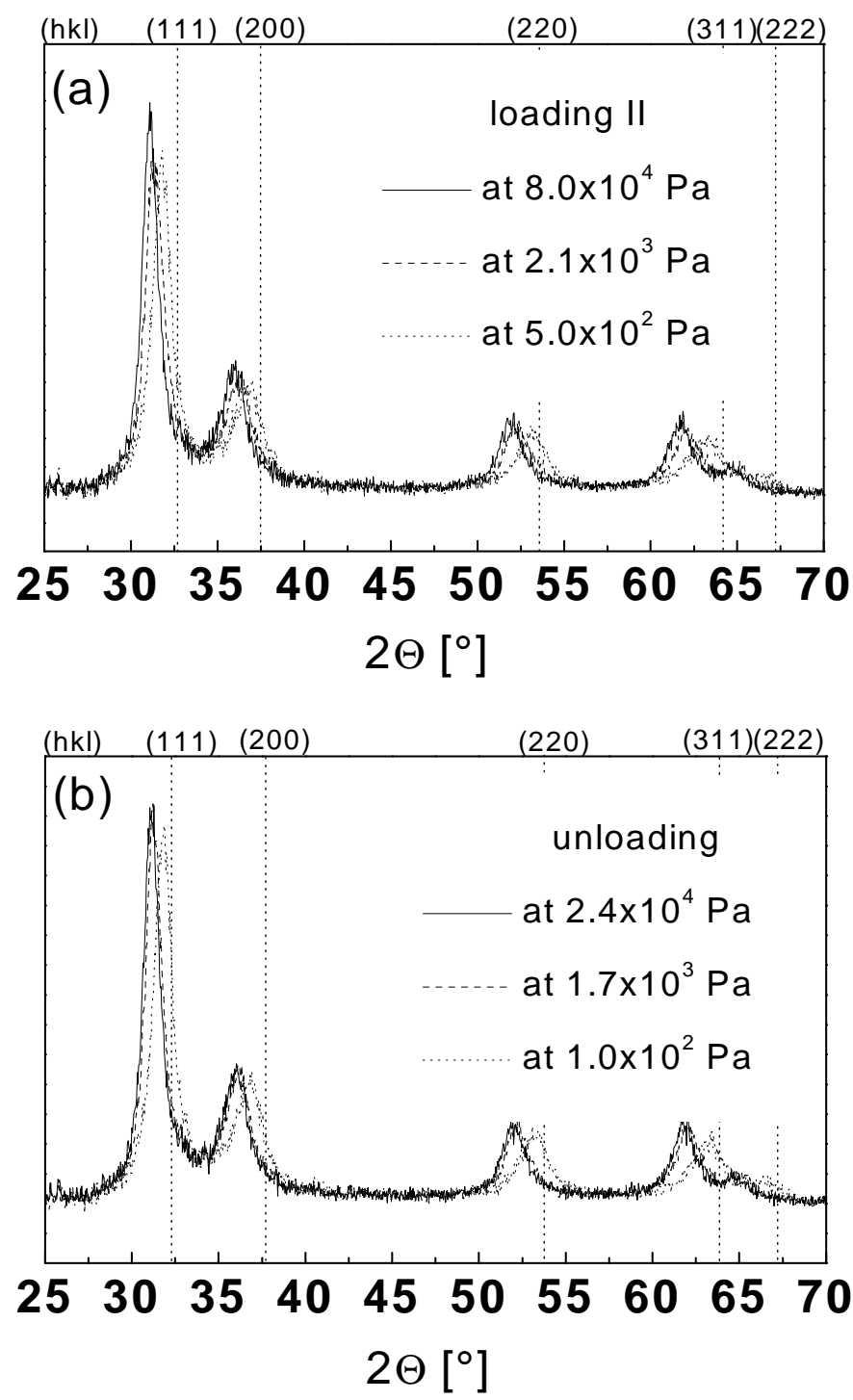

Figure 6. 11. Diffraction patterns of the $5.0 \mathrm{~nm}$ cluster sample: during hydrogen loading (a) and during hydrogen unloading (b):as the hydrogen pressure increases the peaks positions shift to lower $2 \theta$ values. Fig. $6.10(a)$, shows the diffraction patterns three different hydrogen pressures during the second loading cycle: at $5.0 \times 10^{2} \mathrm{~Pa}$ (dotted line), $2.1 \times 10^{3} \mathrm{~Pa}$ (dashed line) and $8.0 \times 10^{4} \mathrm{~Pa}$ (solid line). Only a peaks shift is visible with a slight increase of peaks intensity. Fig.6.10(b) shows the XRD

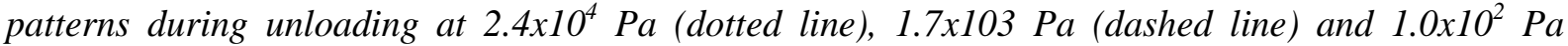
(dotted line) the peaks position shifts to higher $2 \theta$ values. The vertical dotted lines are position of peaks for the fcc bulk Pd at $\lambda=1.2438 \AA$. 
In contrast to the larger clusters $(6.0 \mathrm{~nm}$ and $5.6 \mathrm{~nm})$ this cluster sample does not show the $(\alpha-$ $\alpha^{\prime}$ )-phase transition which is known from $f c c$ bulk Pd. Only a shift of peaks was visible by hydrogen absorption. Thus the possibility of an $\left(\alpha-\alpha^{\prime}\right)$-phase transition was checked by looking for a peak broadening during hydrogen absorption. Peak broadening, if it exist, can be correlated with passing through a two-phase region, because the overlapping of each pair of reflections (resulting from the $\alpha$ - and $\alpha^{\prime}$-phases) will results in peak broadening. Figure 6.12 (a-b) shows the results of fitting the lower angle peak with Lorentzian function. The peak width (near (111) bulk) stays constant at the beginning and then decreases in the pressure range $1.5 \cdot 10^{3}-2.5 \cdot 10^{3} \mathrm{~Pa}$, Fig $6.12 \mathrm{a}$. As the hydrogen pressure increases the peak width stays constant. During hydrogen desorption the peak width stays constant until a hydrogen pressure of $1.0 \cdot 10^{3} \mathrm{~Pa}$ is reached then it start to increase. At the end of the unloading cycle the peak width is narrower than it was in the beginning of the loading cycle. A similar behaviour was also observed for the peak area, Fig. 6.12b. During hydrogen absorption the peak area stays constant at first then it increases at the pressure range $1.5 \cdot 10^{3}-2.5 \cdot 10^{3}$ after that increasing the hydrogen pressure does not affect the peak area. Even though no additional reflections are visible peak width and peak area changes always appear.
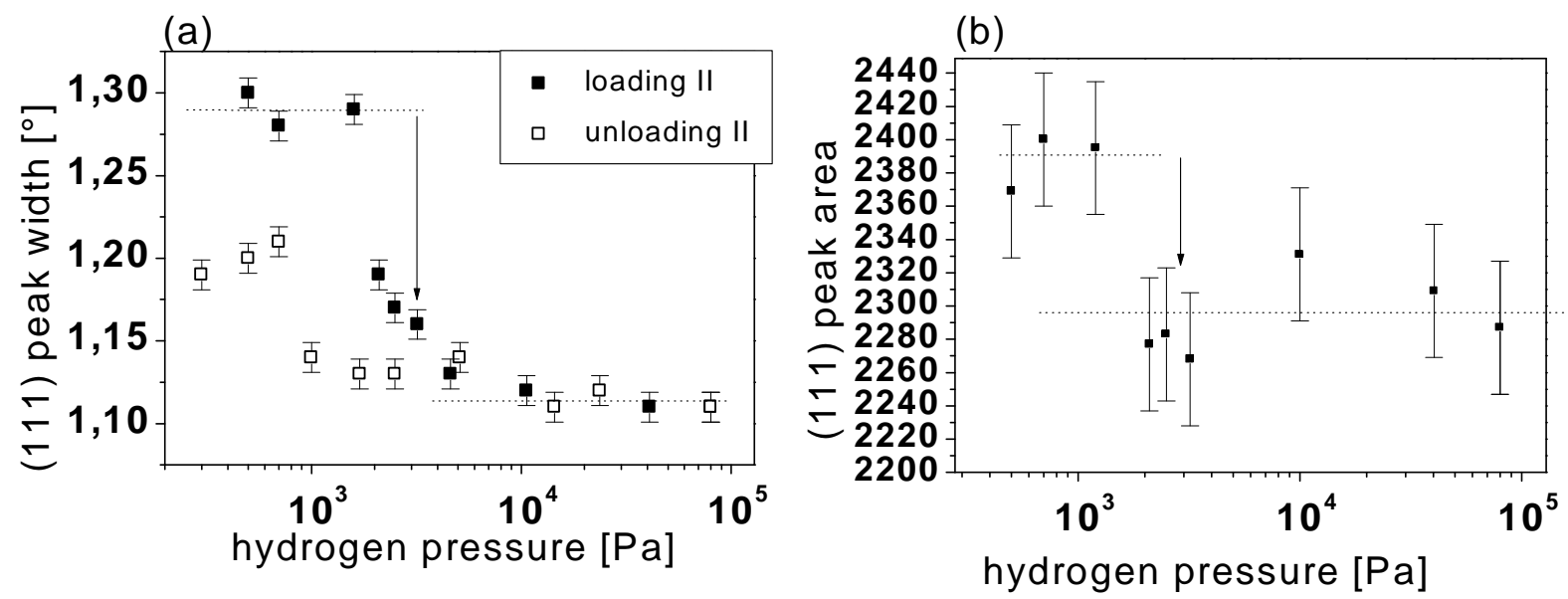

Figure 6. 12. Results of fitting the lower angle peak (111) with Lorenzian function. Fig.6.12a shows the peak width as a function of the hydrogen pressure( logarithmic scale). During loading the width decreases at the pressure range $1.5 \cdot 10^{3}-2.5 \cdot 10^{3} \mathrm{~Pa}$, while during unloading the peak width starts to increase at $1.0 \cdot 10^{3} \mathrm{~Pa}$. After unloading the peak width does not retains it staring value. Fig. 6.12b shows that during loading the peak area decreases at the pressure range $1.5 \cdot 10^{3}-2.5 \cdot 10^{3} \mathrm{~Pa}$. 


\subsubsection{Hydrogenation of $4.8 \mathrm{~nm}$ clusters}

Figure 6.13 shows the XRD patterns of the $4.8 \mathrm{~nm}$ cluster sample during hydrogen loading at different hydrogen gas pressures. As the hydrogen pressure increases the peaks position are shifted to lower $2 \theta$ values, indicating the lattice expansion. Moreover, the maximum intensity of the peaks (peaks height) also increase with increasing the hydrogen pressure, Fig.6.13(a). This behaviour is also observed in a second loading cycle. Loading with hydrogen causes a lattice expansion and an increase in the peaks height. Again, no additional sets of Bragg reflections can be detected. The features found in this cluster sample are similar to those found in the $5.0 \mathrm{~nm}$ clusters sample.

Figure 6.13(b) shows the diffraction patterns during hydrogen unloading. Hydrogen desorption lead to a lattice expansion, the peaks are shifted to higher $2 \theta$ values. On the other hand, the peaks height decreases as the hydrogen pressure decreases.

In this cluster sample only peaks shift was visible by hydrogen absorption. Thus the possibility of an $\left(\alpha-\alpha^{\prime}\right)$-phase transition was checked by looking for a peak broadening during hydrogen absorption. Figure 6.14 (a-b) shows the results of fitting the lower angle peak with a Lorentzin function. The peak width decreases with increasing the hydrogen pressure, Fig. 6.13 (a) solid squares while the integrated peak area stays constant, Fig. 6.14(b). During hydrogen desorption the peak width increases, Fig. 6.14 (a) opened up-triangle.

The XRD pattern of the as-prepared of this cluster sample, on one hand, does not give a direct hint on an icosahedral structure. On the other hand, the relative peaks intensities are not those expected from cubic structure. HREM analysis show that more than $80 \%$ of the particles have icosahedral structure $12 \%$ are multi-twinned and the rest are cubic. This can explain the untypical icosahedral diffraction pattern for this clusters size. The size distribution of this clusters sample is relatively large $( \pm 0.8 \mathrm{~nm})$. Thus we expect that the diffraction pattern is dominated by the larger cubic particles. This will result in untypical icosahedral diffraction pattern although most of the particles within this cluster size have icosahedral structure or are multi-twinned. 

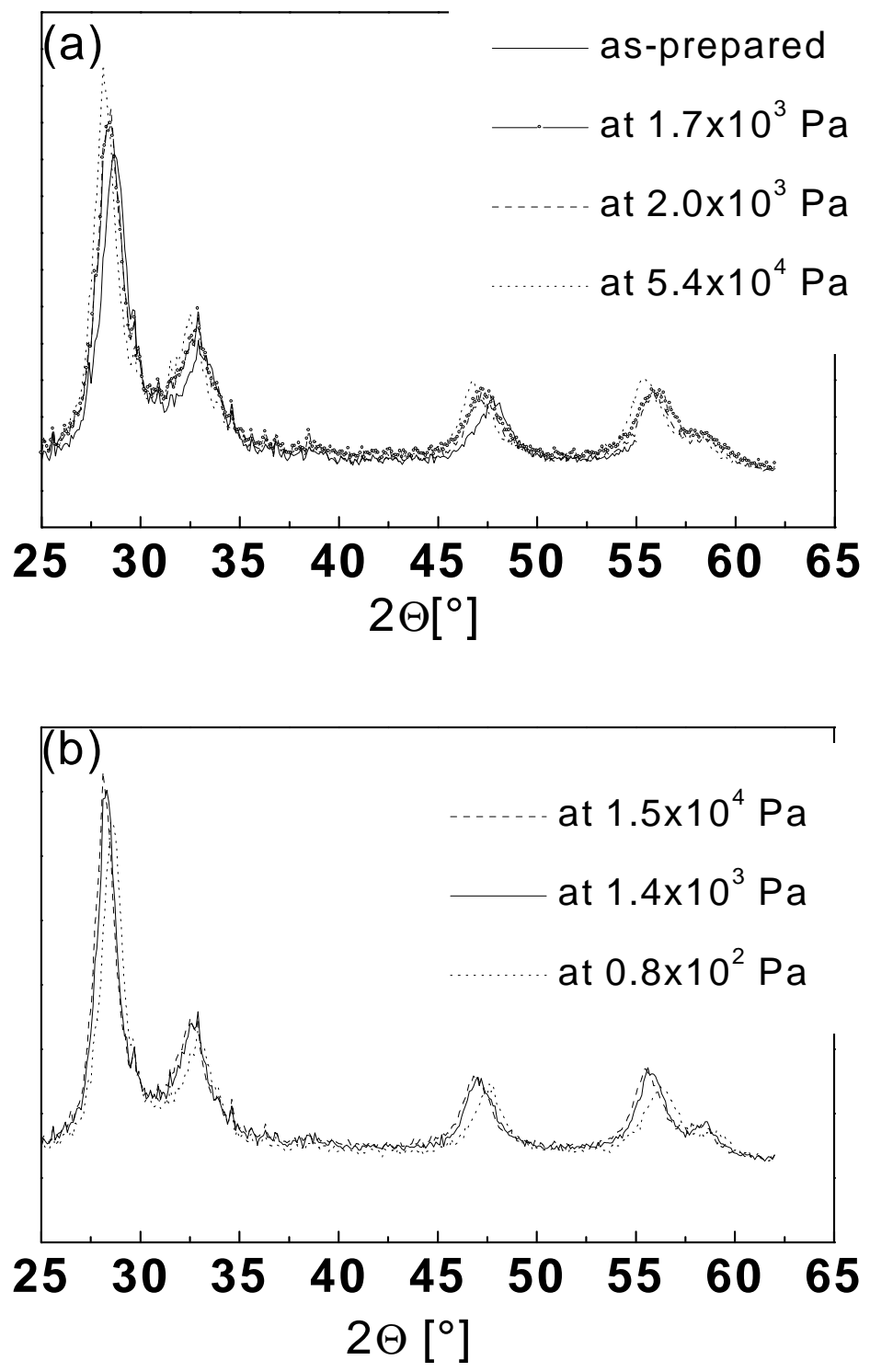

Figure 6. 13. Diffraction patterns of the $4.8 \mathrm{~nm}$ cluster sample at different hydrogen pressures. Fig.6.13a during hydrogen loading: the peaks positions are shitted to lower $2 \theta$ values with increasing the hydrogen pressure and the maximum intensities of the peaks is increased. Fig 6.13b shows the $X R D$ patterns during unloading: the peaks positions are shitted to higher $2 \theta$ values with decreasing the hydrogen pressure, also the peaks height decreases. $\lambda=1.12617 \AA$ 

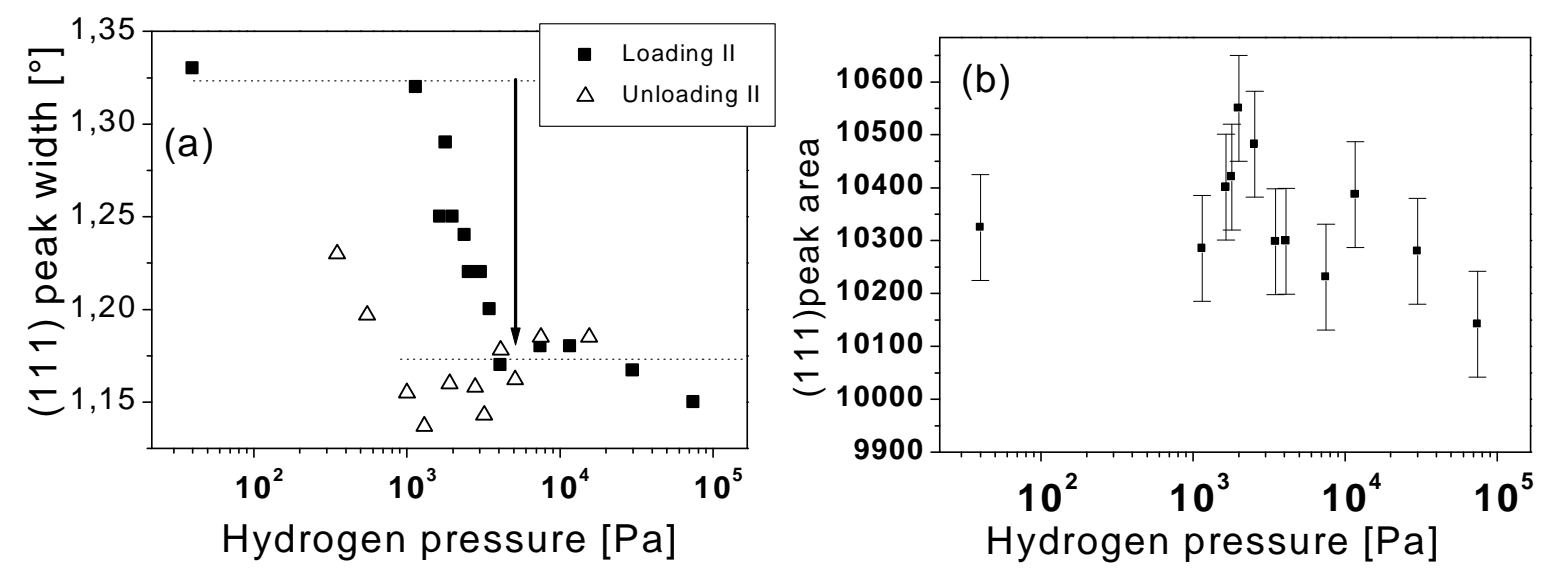

Figure 6. 14. Results of fitting the (111) peak with a Lorentzian function. The peak width (a) and peak area as a function of the hydrogen pressure (logarithmic scale). (a) During loading the width decreases with increasing the hydrogen pressure (solid squares), while during unloading the peak width increases as hydrogen pressure is decreased (triangles). (b) the peak area during hydrogen loading. 


\subsubsection{Hydrogenation of $3.6 \mathrm{~nm}$ clusters}

Figure 6.15 shows the XRD patterns of the $3.6 \mathrm{~nm}$ cluster at different hydrogen gas pressures in comparison with the as-prepared state in the $2 \theta$ range of $25^{\circ}$ to $45^{\circ}$. The structure of this cluster sample was discussed in section (5.4): XRD and HREM analyses show that the asprepared sample has, mainly, an icosahedral structure. This is also visible in the shape of the lower angle lattice reflection.

Fig. 6.15 shows that, increasing the hydrogen pressure leads to a very small dilatation of the lattice, shifting the Bragg reflections to smaller $2 \theta$ values. Although the peak shift is very small, it is obvious that the peaks shift increases with increasing the hydrogen pressure. This is visible by following the bars marking the lowest angle peak positions (near bulk (111) peak) in Figure 6.15.

No phase transition was found in this cluster sample, as shown in Figure 6.15. Moreover, there is no change in the peak intensity.

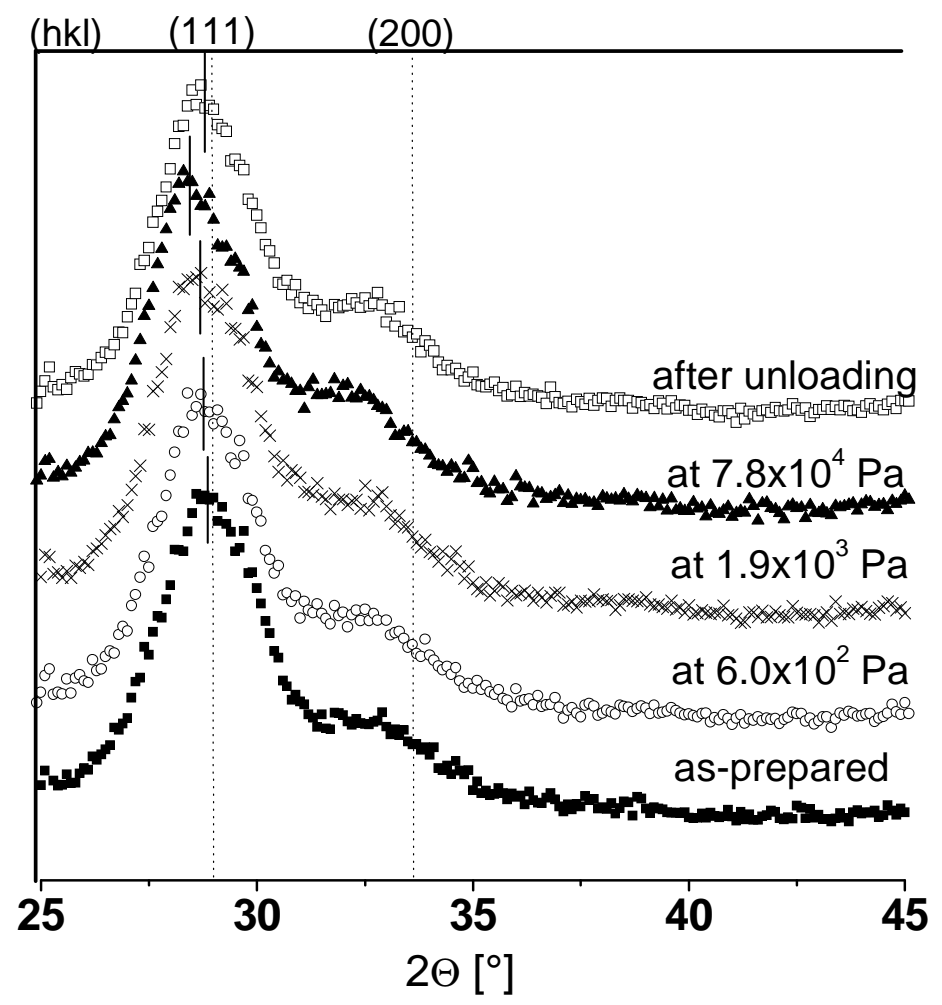

Figure 6. 15. XRD patterns of the $3.6 \mathrm{~nm}$ cluster sample at four different hydrogen pressures monitoring the lattice expansion: Diffraction pattern of as-prepared cluster (after pre-treatment) and at different hydrogen pressures. Only a peak shift is visible. The (111) peak position has been marked with vertical bares. $\lambda=1.24438 \AA$ 


\subsubsection{Hydrogenation of $3.0 \mathrm{~nm}$ clusters}

Figure 6.16 shows the XRD patterns of the $3.0 \mathrm{~nm}$ cluster sample at different hydrogen pressures. Increasing the hydrogen pressure leads to very small shift in the peaks position toward lower $2 \theta$ values. This shift becomes more visible with increasing the hydrogen pressure. On the other hand, no phase transition was observed as shown in Fig. 6.16. There is also no change in the peak intensity. The features observed in this clusters size are similar to those observed in the clusters 3.6nm diameter shown before.

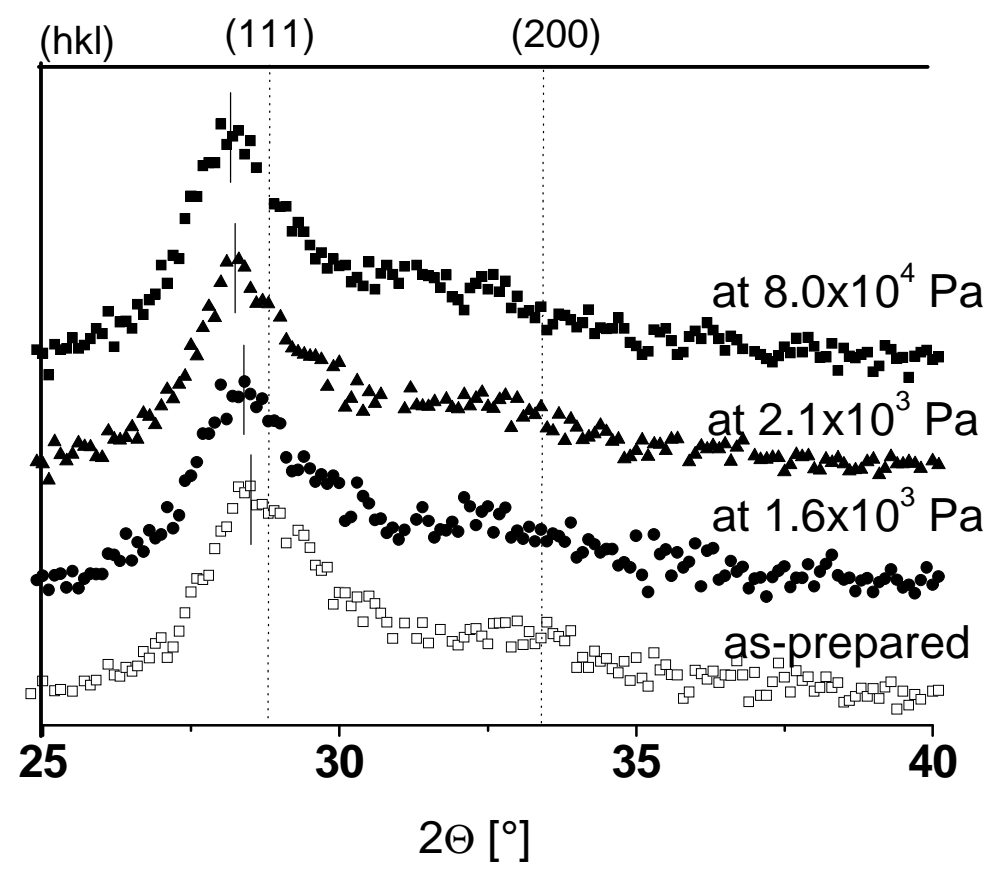

Figure 6. 16. Diffraction patterns of the $3.0 \mathrm{~nm}$ cluster sample at four hydrogen pressures monitoring the lattice expansion: Only a small peak shift is visible. The (111) peak positions have been marked with vertical bars. The vertical dotted lines are the positions of peaks from bulk Pd at $\lambda=1.120599 \AA$ 


\section{Discussion: Pd-H Clusters}

In this chapter the results of the interaction of hydrogen with Pd clusters, presented in the previous chapter, will be discussed. First, lattice changes and pressure-lattice parameter isotherms obtained from the in-situ XRD measurements during hydrogen loading-unloading will be considered. This will be followed by discussing the pressure-concentration isotherms obtained from the gravimetric measurements. The hydrogen solubility and the presence of the two-phase field, as well as, the hysteresis will be interpreted finally.

\subsection{Lattice expansion}

Figure 7.1 (a-f) shows the lattice parameter of different cluster samples as a function of the hydrogen partial pressure during the in-situ XRD measurements. The lattice parameter of each cluster sample was calculated from the position of the lower angle peak (near bulk (111) peak). The lattice expansion was found to be strongly size dependent. The lattice expansion value for larger clusters is higher than those for smaller ones, but still smaller than those for bulk Pd. For example, after loading cycle, $\Delta \mathrm{a}=0.123 \AA$ for the $6.0 \mathrm{~nm}$ cluster but $\Delta \mathrm{a}=$ $0.036 \AA$ for the smallest cluster $(3.0 \mathrm{~nm})$.

The pressure-lattice parameter isotherm for the $6.0 \mathrm{~nm}$ cluster is shown in Fig.7.1(a). The plot shows that for regions where there is a gradual increase in the equilibrium hydrogen pressure with each dose of hydrogen, there is also a complementary gradual increase in the lattice parameter (over a region designated $\alpha$-phase in the pressure-concentration isotherm). However, when a pressure of $2 \cdot 0 \cdot 10^{3} \mathrm{~Pa}$ is reached, the lattice parameter increases strongly. This region will be called the "lattice parameter miscibilty gap" and it is comparable to the miscibility gap found in the pressure-concentration isotherms. This strong increase in the lattice parameter in this region corresponds to the appearance of the ( $\alpha^{\prime}-\mathrm{Pd}-\mathrm{H}$ phase) hydride phase as we have seen in section (6.2.1). Finally, when the $\alpha$-phase disappears, corresponding 
to the vanishing of the $\alpha-\mathrm{Pd}-\mathrm{H}$ phase reflections as shown in (6.2.1), a further gradual increase in the lattice parameter is observed indicating a further expansion of the hydrided $\mathrm{Pd}$ cluster. On reversing the sequence and now unloading hydrogen from the cluster, there is first an apparent reversibility of plots of lattice constant against pressure besides a hysteresis at the $\alpha-\alpha^{\prime}$ phase transition. As the pressure decreases further the lattice parameter decreases and then decreases strongly at $1.0 \cdot 10^{3} \mathrm{~Pa}$. The same behaviour was also observed during the hydrogenation of the $5.6 \mathrm{~nm}$ cluster sample, see Fig.7.1(b). The lattice parameter isotherm obtained for this cluster show that during hydrogen loading the lattice parameter increases strongly in the pressure range $1.8 \cdot 10^{3}-2.5 \cdot 10^{3} \mathrm{~Pa}$ and during unloading decreases strongly in the pressure range $1.8 \cdot 10^{3}-1.2 \cdot 10^{3} \mathrm{~Pa}$. The larger clusters $(6.0 \mathrm{~nm}$ and $5.6 \mathrm{~nm})$ show a typical lattice-parameter relation as that obtained from the hydrogen absorption by Pd-powder $(f c c$ structure) shown in chapter 1 (Fig.1.2).

The pressure-lattice parameter isotherms of the $5.0 \mathrm{~nm}$ cluster and the $4.8 \mathrm{~nm}$ cluster samples are shown in Fig.7.1(c) and (d), respectively. The cluster samples also show that the lattice parameter values increase strongly in the pressure range of $1.2 \cdot 10^{3}$ to $2.0 \cdot 10^{3} \mathrm{~Pa}$ during $\mathrm{H}$ loading and a decrease in the range of $1.0 \cdot 10^{3}$ to $6.0 \cdot 10^{3} \mathrm{~Pa}$ during unloading. This strong lattice parameter change shows a plateau like region (lattice parameter miscibility gap). This indicates that a transition is occurring in this pressure range although the XRD pattern do not show an $\left(\alpha-\alpha^{\prime}\right)$-phase transition as we have seen in sections (6.2.3) and (6.2.4). Moreover, no peak broadening was observed during hydrogen loading. Instead the peaks width becomes narrower during hydrogen loading and broader during hydrogen unloading as have been shown in sections (6.2.3) and (6.2.4). Thus the transition found in these cluster samples could not be interpreted as the common $\left(\alpha-\alpha^{\prime}\right)$-phase transition.

A continuous slight linear increase in the lattice parameter of the $3.6 \mathrm{~nm}$ cluster sample and the 3.0 samples with increasing the H-pressure was found, Fig.7.1(e) and Fig.7.1(f) respectively. No lattice parameter miscibility gap was found for these cluster sizes. This indicates a narrowing of the lattice parameter miscibility gap on going from larger clusters to smaller ones. Also, this is in accordance with the narrowing of the miscibility gap in the pressure-concentration isotherms found in these clusters (see the next section). Narrowing of the miscibility gap, in the pressure-concentration isotherms, was observed by other workers on going from bulk Pd to nanometer sized polycrystalline materials $[36,7,8,10]$.

XRD and HREM analyses of these cluster samples show that: the larger clusters $(6.0 \mathrm{~nm}$ and $5.6 \mathrm{~nm}$ ) have predominately a cubic lattice structure, where smaller clusters $(5.0 \mathrm{~nm}, 4.8 \mathrm{~nm}$, $3.6 \mathrm{~nm}$ and $3.0 \mathrm{~nm}$ ) have predominately an icosahedral structure (in the as-prepared state). The 
different lattice structure found in the smaller clusters should be taken into account when discussing an $\left(\alpha-\alpha^{\prime}\right)$ phase transition. The $\left(\alpha-\alpha^{\prime}\right)$ phase transition occurred in bulk Pd. It is known that, for bulk Pd, both the $\alpha-\mathrm{Pd}-\mathrm{H}$ phase and the $\alpha^{\prime}-\mathrm{Pd}-\mathrm{H}$ phase (hydride phase) have a face-centred-cubic $(f c c)$ structure with hydrogen occupying the $O_{h}$ interstitial sites in the $\mathrm{Pd}$ lattice. This was found to be the case in the larger cubic clusters $(6.0 \mathrm{~nm}$ and $5.6 \mathrm{~nm})$, where in-situ XRD measurements confirms the $\left(\alpha-\alpha^{\prime}\right)$ phase transition during hydrogen loading and the $\left(\alpha^{\prime}-\alpha\right)$ phase transition during unloading. In the smaller icosahedral clusters $(5.0 \mathrm{~nm}, 4.8$ $\mathrm{nm}, 3.6 \mathrm{~nm}, 3.0 \mathrm{~nm}$ ) the case is different and we believe that the sloped plateau like region found in the pressure-lattice parameter isotherms in the $5.0 \mathrm{~nm}$ and $4.8 \mathrm{~nm}$ cluster could not be due to the $\left(\alpha-\alpha^{\prime}\right)$ phase transition known for $f c c$ structured Pd. In the in-situ XRD measurements of these cluster changes in the diffractogram were observed during hydrogen loading-unloading, such as: First, peaks narrow during hydrogen loading and broadening during unloading. Second, peaks height change in the $4.8 \mathrm{~nm}$ cluster sample where the peaks height increases during hydrogen loading and decrease during unloading. Third, the $5.0 \mathrm{~nm}$ cluster as-prepared(not having been exposed to hydrogen) shows a dramatic change in the diffractogram after the exposure of the sample to hydrogen gas: all the peaks become narrower and the intensity increases. The cluster diffractogram does not retrain its original shape after the removal of hydrogen from the sample. Thus we attribute the sloped plateau region found in the $5.0 \mathrm{~nm}$ cluster to be due to a structural transition. Most probably this is from the icosahedral to the cubic lattice structure (ico $\rightarrow$ cub). This (ico $\rightarrow$ cub) transition seems to be reversible for the $4.8 \mathrm{~nm}$ cluster and is not completely reversible for the $5.0 \mathrm{~nm}$ cluster. In the $5.0 \mathrm{~nm}$ cluster samples, although it is observed that the structural change is occurring in the pretreatment step this could not excludes the possibility of a further (ico $\rightarrow$ cub) transition during hydrogen loading resulting from non-transformed icosahedral particles.

The absorption pressure-lattice parameter isotherms for all cluster samples show the presence of a hysteresis. This may be evidence for the existence of a phase transition even in the (3.6 $\mathrm{nm}, 3.0 \mathrm{~nm})$ smallest clusters. In these $(3.6 \mathrm{~nm}, 3.0 \mathrm{~nm})$ clusters no changes in the XRD patterns during hydrogen loading-unloading were observed, also, no plateau like region was found in the pressure-lattice parameter isotherms. The phase transition in these clusters can be, also, interpreted as an (ico $\rightarrow$ cub) transition in which only the larger particles within this cluster size range participate in the transition and the smaller particles do not contribute to this transition and prefer to maintain their icosahedral structure. Also, for small clusters, the assumption that the transition occurs between icosahedral phases could not be excluded. 
(a) $6.0 \mathrm{~nm}$ cluster
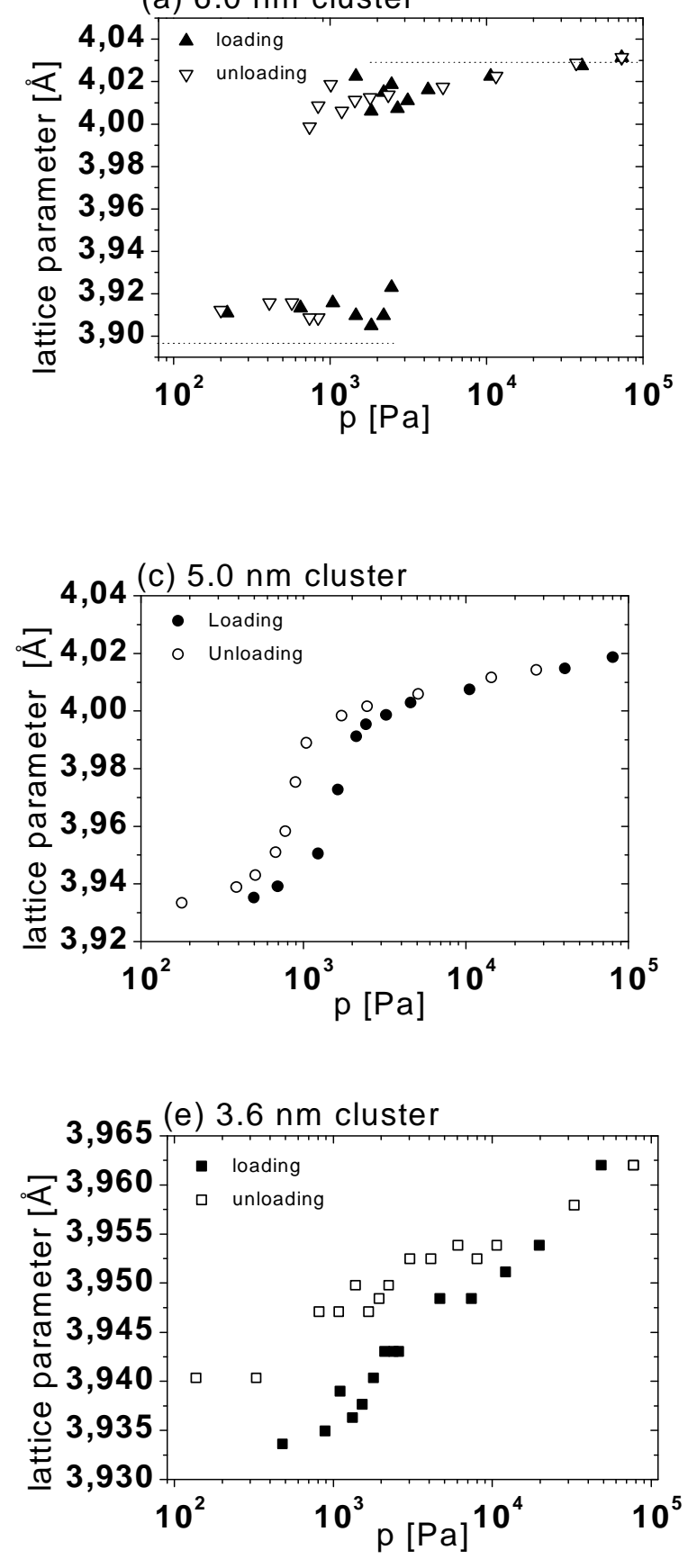

(b) $5.6 \mathrm{~nm}$ cluster
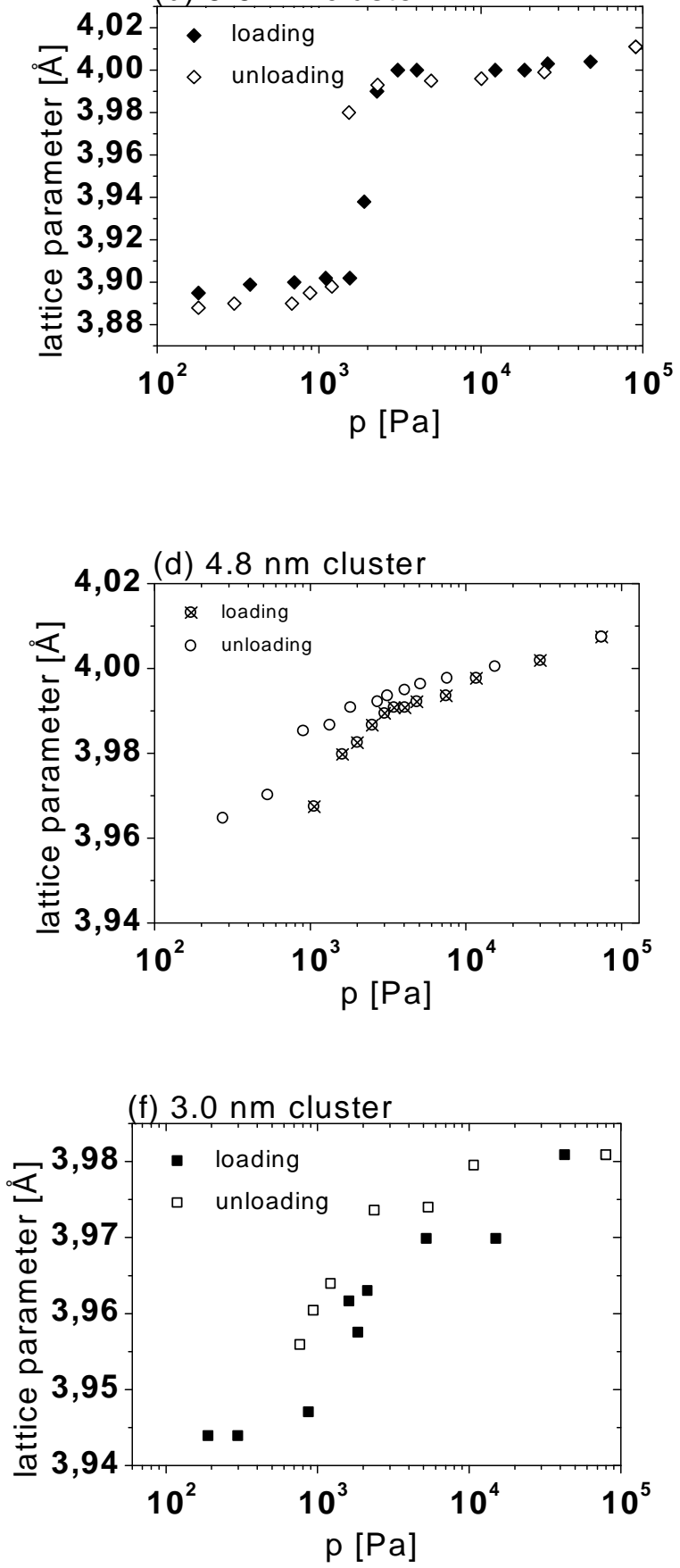

Figure 7. 1 The lattice parameter of different Pd cluster samples at different hydrogen pressures: during hydrogen loading and during hydrogen unloading. The dotted line in 7.1(a) represent the Pd bulk values. The lattice expansion of the cluster is strongly size dependent. Larger clusters have higher lattice parameter values in comparison to smaller ones at the same hydrogen pressure. Please notice the different scaling of the ordinates. 


\subsection{Pd-H Cluster Isotherms}

The pressure-concentration isotherms for different cluster sizes and Pd-powder, in the absorption direction, are shown in Figure 6.2. Absorption-desorption isotherms are shown also in Figure 7.2. The most remarkable features of these isotherms when compared to Pd bulk are: First, the enhanced hydrogen solubility in the $\alpha$-phase. Second, the narrowing in the two-phase region (miscibility gap), see Table 7.1. Third, in all the isotherms a hysteresis is visible.

\subsubsection{Existence of the two-phase region}

As shown in chapter 1 , the Pd bulk isotherm typically contains a region where there the pressure does not depend on concentration. In this region the isotherm shows a plateau. This, generally non-sloped, plateau represent the two-phase region or the miscibility gap between the $\alpha$-phase and $\alpha^{\prime}$-phase. In the $f c c$ structured bulk Pd the presence of the two-phase region is regarded as evidence for the $\left(\alpha-\alpha^{\prime}\right)$ phase transition.

Figure 6.2a shows the $p-x-T$ isotherm for the Pd-powder. The isotherm shows a plateau around the absorption pressure of $2.0 \cdot 10^{3} \mathrm{~Pa}$ indicating the existence of the $\left(\alpha-\alpha^{\prime}\right)$ phase transition.

Absorption isotherms for different cluster sizes are shown in Fig. 6.2. Absorption-desorption isotherms of these cluster samples are shown also in Figure 7.2. The isotherms of the larger clusters $(5.2 \mathrm{~nm}, 4.8 \mathrm{~nm}$ and $3.6 \mathrm{~nm})$ show sloped plateaus. Whereas, the isotherm of the smallest $(3.0 \mathrm{~nm})$ cluster do not show such a plateau. Thus, in the larger clusters a two-phase region exists. All the absorption-desorption isotherms of the different clusters show a hysteresis, Fig. 7.2. The hysteresis is also, a piece of evidence for the phase transition [28]. Thus, the hysteresis shown in all cluster samples isotherms verifies the existence of the twophase field even for smallest $(3.0 \mathrm{~nm})$ cluster sample. However, as we have seen in the previous sections, the presence of the two-phase field in these cluster samples does not imply that an $\left(\alpha-\alpha^{\prime}\right)$ phase transition take place, because the cluster samples in this size range have a different structure than the cubic one. In section (5.4) we have seen that clusters smaller than $5.6 \mathrm{~nm}$ have mainly an icosahedral structure. The two-phase field found in these cluster sizes is therefore attributed to a (ico $\rightarrow$ cub) transition as we have seen in the previous section. 

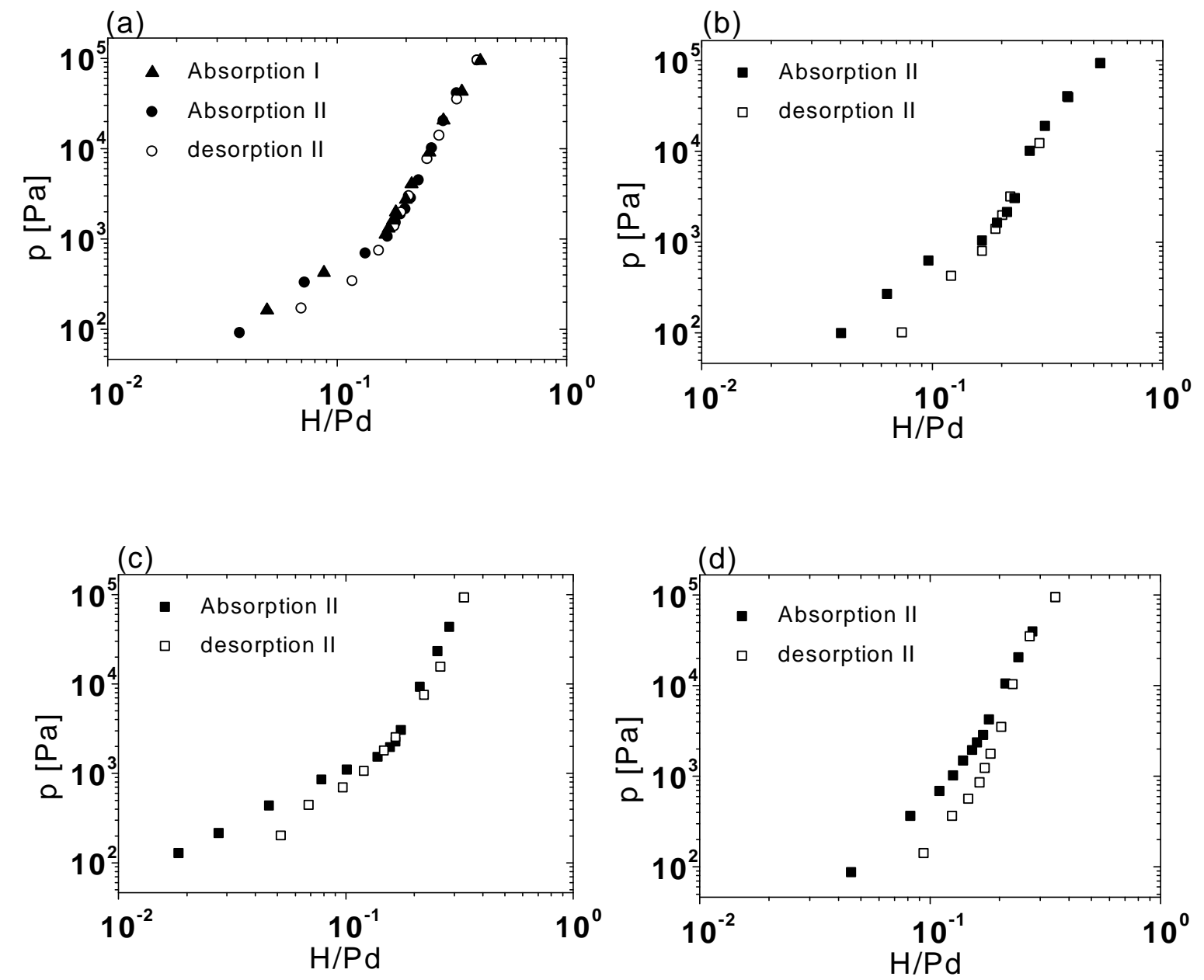

Figure 7. 2. Isotherms of Pd cluster samples in absorption and desorption direction: $5.2 \mathrm{~nm}$ cluster sample Fig.7.2(a), $4.8 \mathrm{~nm}$ cluster sample Fig. 7.2(b), $3.6 \mathrm{~nm}$ cluster sample Fig. 7.2(c) and $3.0 \mathrm{~nm}$ cluster sample Fig. 7.2(d). 


\subsubsection{Solubility and the width of the two-phase region}

The solubility of all Pd-H cluster samples, in the low concentration range, shows a significant increase in comparison with bulk Pd. The absorption isotherm for Pd-powder shows a typical slope $(d)$ of 2 at low concentrations as expected from Sieverts' law, where $(d=\partial \ln p / \partial \ln x)$. The slope for the cluster sample is: 1.6 for the $5.2 \mathrm{~nm}$ cluster, 2.0 for the $4.8 \mathrm{~nm}$ cluster, 1.3 for $3.6 \mathrm{~nm}$ cluster and 2.2 for the $3.0 \mathrm{~nm}$ cluster. A typical slope of 2 at low hydrogen concentration means that the hydrogen absorption is a second order reaction i.e. the hydrogen molecules dissociated at the surface. The deviation from the ideal slope found in this work can be attributed to: First, the presence of $\mathrm{H}-\mathrm{H}$ interaction even at low hydrogen concentration yielding a slope of at less than 2. In Sieverts' law one assumes that such interaction does not occur at low pressure. Second, uncertainty arising from the few number of data points at low concentration due to the limitation of the measurement method. Difficulties in determining the slope was also reported by other authors. Züttel et al. [10] reported a slope of 4 for $3.8 \mathrm{~nm} \mathrm{Pd}$ cluster. They attributed that to the spatial limitation of the Pd cluster. Sachs et al. [8] reported a slope of one for $5.0 \mathrm{~nm}$ cluster. This was interpreted as a molecular hydrogen absorption rather than dissociative absorption. In this work, the order of reaction is close to 2 this implies a dissociative hydrogen absorption.

The solubility increase compared to bulk Pd can be attributed to the additional absorption in the surface and subsurface sites. The percentage of the surface atoms varies from about $50 \%$ to $20 \%$ in the cluster size range of 2 to $6 \mathrm{~nm}$, see section (2.1.2). The $\mathrm{H}$ is bound strongly to the surface at room temperature while it could not leave the surface. However, it can leave the subsurface sites even at room temperature [86]. The cluster samples were pre-treated with hydrogen in order to remove any oxide. Thus most of the surface sites are expected to be saturated with hydrogen and some are blocked due to the surfactant shell. Therefore, we assume that the H-atoms will occupy the subsurface and inner-sites (bulk site). The assumption of additional occupation of the subsurface sites was used by Pundt et al. to explain the increased $\alpha$-phase solubility [7].

The width of the plateau (miscibility gap) of the Pd-H phase boundary exhibits significant narrowing with decreasing the cluster size(cf. Table 7.1). For the $3.0 \mathrm{~nm}$ cluster it is not clear yet if a miscibility gap is still present (see Figure 7.2). The maximum $\alpha$-phase solubility limit 
increases while the minimum $\alpha^{\prime}$-phase solubility decreases in comparison to bulk. Similar results were obtained for nanocrystalline Pd. Mütschele and Kirchheim [4,36] and Estman et al. [6] have shown that the width of the plateau (miscibility gap) exhibits significant narrowing, for nanocrystalline samples as compared to that of the conventional polycrystalline samples. Similar results were observed for nanometer-sized clusters [7-9]. In an electrochemical hydrogen loading study of small Pd clusters (about $1.2 \mathrm{~nm}$ diameter which were embedded in $\mathrm{Cu}$-powder), Nützenadel et al. [9] found that it was not clear if the $1.2 \mathrm{~nm}$ cluster exhibited a phase transition. Nützenadel et al. explained this by a lowering of the critical temperature $\left(\mathrm{T}_{\mathrm{c}}\right)$ of the $\alpha-\alpha^{\prime}$-miscibility gap below room temperature, resulting in no phase transition for this cluster size. In addition, calculations by Wolf et al. [15] showed that, for small $\mathrm{Pd}$ clusters (500 $\mathrm{Pd}$ atoms with about $2.6 \mathrm{~nm}$ diameter), no phase transition is expected. These calculations were based on a cubic lattice structure.

In nanocrystalline $\mathrm{Pd}$, the increased $\alpha$-phase solubility was attributed to the enhanced hydrogen solubility in the grain boundaries while the decreased $\alpha^{\prime}$-phase solubility was explained by the assumption that no phase transition occurs in the grain boundaries. Mütschele and Kirchheim [4] described a model that determine the fraction of sites that do not participate in the hydride formation. This two-site model was applied by Pundt et al. [7] in $\mathrm{Pd}$ clusters whereby $\mathrm{H}$-atoms can only occupy subsurface or bulk-sites. Assuming that the hydrogen in subsurface sites does not transform to a hydride phase the fraction $c_{t}$ of subsurface sites $N_{s s}$ using the phase boundaries of the two-phase region is:

$$
c_{t}=\frac{N_{s s}}{N}=1-\frac{c_{\alpha^{\prime}}^{c l}-c_{\alpha}^{c l}}{c_{\alpha^{\prime}}^{p o l y}-c_{\alpha}^{p o l y}}
$$

with $c_{\alpha^{\prime}}^{\text {poly }}=0.607 \mathrm{H} / \mathrm{Pd}$ and $c_{\alpha}^{\text {poly }}=0.008 \mathrm{H} / \mathrm{Pd}$. 
Pundt et al. and Sachs et al. also determined $c_{t}$ theoretically. Assuming that the two outer shells do not participate in the phase transition the calculated $c_{t}$ values and the experimental one were found to be in a good agreement for the $2 \mathrm{~nm}$ and the $3 \mathrm{~nm}$ Pd clusters. Theoretical modelling of hydrogen solubility in Pd cluster was performed recently by Srivastava and Balasubramaniam [87]. This study revealed that the subsurface site energy is lower than the inner (bulk) sites energy and that the subsurface sites are preferentially filled up by hydrogen before the inner sites get filled.

Applying the two-site model to the result of this work we get: $c_{t}=81 \%$ for the ms 706 cluster, $c_{t}=80 \%$ for the ms 718 cluster and $c_{t}=86 \%$ for the msE18 cluster. The $c_{t}$ values found in this work suggest that the three outer shells in the Pd cluster do not participated in the phase transition. In this work we assume that the number of the subsurface sites corresponds to the number of the Pd atoms in the three outer shells. Utilising the atomic shell structure ( see section (2.4.2) and Table 2.1) where the number of atom in the shell $n$ is $N_{n}=10 n^{2}+2$ and based on this assumption the theoretical $c_{t}$ values are: for the $5.2 \mathrm{~nm}$ cluster $c_{t}=72 \%$ (11 shells cluster, $\left.N=5083, N_{11}=1442, N_{10}=1212, N_{9}=1002\right)$. For the $4.8 \mathrm{~nm}$ cluster $c_{t}=78 \%$ (10 shells cluster, $N=3871, N_{10}=1212, N_{9}=1002, N_{8}=812$ ). For the $3.6 \mathrm{~nm}$ cluster $c_{t}=80 \%$ ( 8 shells cluster, $N=2057, N_{8}=812, N_{7}=492, N_{6}=362$ ). The calculated $c_{t}$ values are in good agreement with the experimental data.

Table 7. 1 Concentration limits of the two-phase region for different cluster sizes during Absorption and the mean plateau pressure.

\begin{tabular}{|c|c|c|c|c|c|c|}
\hline Sample & Size & \multicolumn{3}{|c|}{ Absorption } & $\begin{array}{c}\text { Desorption } \\
p_{\text {Plateau }}^{\text {des }}[\mathrm{Pa}]\end{array}$ & $\frac{p_{a b}}{p_{\text {des }}}$ \\
\cline { 3 - 7 } & {$[\mathrm{nm}]$} & $\mathrm{c}_{\alpha, \max }[\mathrm{H} / \mathrm{Pd}]$ & $\mathrm{c}_{\alpha^{\prime}, \min }[\mathrm{H} / \mathrm{Pd}]$ & $p_{\text {Plateau }}^{\text {Ab }}[\mathrm{Pa}]$ & & \\
\hline Pd-powder & $400 \mu \mathrm{m}$ & 0.03 & 0.39 & $2.0 \times 10^{3}$ & & 1.95 \\
\hline ms706 & 5.2 & 0.06 & 0.17 & $8.0 \times 10^{2}$ & $4.1 \times 10^{2}$ & 1.75 \\
\hline ms718 & 4.8 & 0.08 & 0.20 & $1.0 \times 10^{3}$ & $5.7 \times 10^{2}$ & 1.83 \\
\hline msE18 & 3.6 & 0.07 & 0.15 & $1.3 \times 10^{3}$ & $7.1 \times 10^{2}$ & \\
\hline
\end{tabular}




\subsubsection{Slope in the two-phase region}

The pressure-concentration isotherms of all cluster samples show a slope in the plateau pressure (a slope in the chemical potential) which do not occur in the bulk $\mathrm{Pd}-\mathrm{H}$ system. The slope increases with decreasing cluster size.

Salomons et al. [88] measured isotherms on $17 \mathrm{~nm}$ and $7.3 \mathrm{~nm}$ Pd clusters deposited on $\mathrm{SiO}_{2}$ and on $\mathrm{Al}_{2} \mathrm{O}_{3}$, respectively. They also found a slope in the two-phase region and explained it by a size dependent shift in the chemical potential due to the surface tension $\gamma$. Samples with a broader distribution of particle sizes were found to have plateaus with a larger slope. For the $7.3 \mathrm{~nm}$ cluster they found that $\gamma=3.15 \mathrm{~N} / \mathrm{m}$. It is worth to mention that this value was obtained at $500 \mathrm{~K}$ and assuming that the particles are limited by the (111) crystallographic planes. Smaller $\gamma$ values were reported by Miedema. From an extrapolation of the surface tension of the liquid at the melting point Miedema predicted that at $0 \mathrm{~K}, \gamma=2.1 \mathrm{~N} / \mathrm{m}$ for Pd. Pundt [89] showed that this contribution can not explain the experimental data, because a surface tension value of $\gamma=0.1 \mathrm{~N} / \mathrm{m}$ is necessary to fit the experimental data of the surfactant stabilised cluster.

Electrochemical H-loading measurements performed by Züttel et al. on a monodisperse narrowly distributed Pd clusters show also a slope in the plateau pressure which decreases with increasing the cluster size $(1 \mathrm{~nm}$ and $2.6 \mathrm{~nm})$. However, the slope in the plateau pressure for the adherent Pd cluster (polymer stabilised) is larger than that for surfactant stabilised Pd cluster. In contrast to the interpretation of Salomons the plateau slope can be explained by an adhesion tension between the cluster particles and the stabiliser.

The effect of the stabiliser on the properties of clusters is a fundamental question. To our knowledge reports on the effect of the stabiliser on the cluster are still very limited. Fu et al. [90] studied the effect of different organic stabiliser on the platinum $4 \mathrm{f}$ binding energy of $\mathrm{Pt}$ clusters. The study was performed on monodispersed cluster $(1.3 \mathrm{~nm})$ stabilised with alkylamine, alkylthiol, $\mathrm{PVP}, \mathrm{PVA}$ or $\mathrm{PPh}_{3}$. It was observed that the $\mathrm{Pt} 4 \mathrm{f}$ binding energies in the same-sized clusters increases by $0.5-0.8 \mathrm{eV}$ relative to those of bulk Pt. Thus, it will be necessary to perform hydrogenation reaction on single-size clusters stabilised in different matrices. Such a study can verify if different stabiliser, with different binding energies as above, will lead to different adhesion tension between the cluster particles and the stabiliser. To our knowledge there are no reports on such study yet. 


\subsubsection{Hysteresis}

Gravimeteric absorption and desorption isotherms are shown in Figure 7.2. It can be seen that all desorption isotherms of the clusters show hysteresis effects. This verifies the existence of a two-phase field for small Pd-H clusters. Gasvolumetric absorption-desorption isotherms and electrochemical absorption-desorption isotherms performed on Pd cluster show the existence of a hysteresis $[7,8,10]$. In bulk Pd-H systems hysteresis was explained by the formation of misfit dislocations [37,38]. This is impossible in the case of clusters. Hysteresis in clusters can be explained by the thermodynamics of an open, coherent two-phase system, see chapter 1. According to Schwartz and Khachaturyan [41] for the absorption $p_{a b}$ and the desorption $p_{d e s}$ plateau pressure the following relation holds:

$$
\ln \left(\frac{p_{a b}}{p_{d e s}}\right)=\frac{4 \Omega G_{s} \frac{1+v}{1-v} \varepsilon_{o}^{2}\left(c_{\alpha^{\prime}}-c_{\alpha}\right)}{k T}
$$

where $\Omega=2.607^{3}$ the volume of one hydrogen atom in $\mathrm{Pd}$, the shear modulus of $\mathrm{Pd} G_{s}=$ $47.4 \times 10^{9} \mathrm{~Pa}$, the Poisson number $v=0.385$, the change in the lattice constant $\varepsilon_{o}=0.063$, and the boundaries of the two-phase region $\mathrm{c}_{\alpha}=0.008, \mathrm{c}_{\alpha}=0.607$, we get a pressure ratio $\frac{p_{a b}}{p_{\text {des }}}=1.86$ for bulk Pd.

The experimentally determined ratios of the measured plateau pressures, see Table 7.1

$$
\left.\frac{p_{a b}}{p_{d e s}}\right|_{m s 706}=\left.1.95 \quad \frac{p_{a b}}{p_{d e s}}\right|_{m s 718}=\left.1.75 \quad \frac{p_{a b}}{p_{d e s}}\right|_{m s E 18}=1.83
$$

are in good agreement with the theory. Thus the hysteresis found in these isotherms can be explained in terms of the coherent phase transformation of an open system. 


\section{Summary}

Nanometer-sized Pd cluster were studied in this thesis. This work was split in to two main parts: First, size selective electrochemical preparation of Pd clusters was investigated. This was followed by studying interaction of hydrogen with palladium clusters from both kinetic and thermodynamic point of view.

The experimental results on the electrochemical preparation of Pd clusters show that:

- Different cluster sizes can be selectively prepared depending on the preparation parameters, such as the current destiny, the electrolysis temperature, the solvent and the distance between the electrodes, as follows:

- Increasing the current density decreases the Pd cluster size. Different cluster sizes in the range 3.0-6.0 $\mathrm{nm}$ (TEM) can be prepared depending on the imposed current density.

- The Pd cluster size increases with increasing temperature of the electrolysis. Clusters in the range of 2.4 to $5.5 \mathrm{~nm}$ (TEM) can be obtained.

- The distance between the electrodes was also found to affect the Pd cluster size.

Cluster sizes in the range of 3.1 to $5.2 \mathrm{~nm}$ (TEM) can be prepared depending on the selected distance between the Pd anode and the cathode.

- Electrolysis in different solvents gives Pd cluster sizes in the range between $3.1 \mathrm{~nm}$ (TEM) in pure $\mathrm{CH}_{3} \mathrm{CN}$ and $2.4 \mathrm{~nm}$ (TEM) in pure THF.

- The electrolysis parameter do not only affect the cluster size but also the size distribution of the cluster. Electrolysis at low temperature gives clusters with narrower size distribution than electrolysis at higher temperature also size distribution of the cluster was found to becomes narrower with the decreased polarity of the solvent.

- The cluster size is best determined by TEM. Determination of the cluster size from the $\mathrm{XRD}$ data is generally affected by the variety of structures and the size dependent nature of the diffraction pattern. It was shown that a Fourier transform of the XRD pattern gives a fairly accurate value of the cluster size. 
- HREM and XRD analysis revealed that the structure of the Pd clusters is size dependent. Clusters smaller than $5.4 \mathrm{~nm}$ were found to have an icosahedral structure where cluster equal to, or larger than, $5.4 \mathrm{~nm}$ were found to have cubic structure.

- The Pd clusters are stabilised with one monomolecular layer of $\mathrm{Oc}_{4} \mathrm{~N}^{+} \mathrm{Br}^{-}$.

The interaction of hydrogen with palladium clusters was studied by in-situ XRD during hydrogen loading-unloading and by performing gravimetric measurements. The results on Palladium-hydrogen clusters are summarised in the following:

- In-situ XRD measurements during hydrogen loading-unloading show that:

- The kinetic of hydrogen absorption is very fast; within 30 min equilibrium is reached in the single phase region.

- The lattice expansion of the Pd clusters is strongly size dependent. Larger clusters show lattice expansion values higher than those of smaller ones but still smaller than those of bulk Pd.

- Large clusters $(\geq 5.4 \mathrm{~nm})$ show an $\left(\alpha-\alpha^{\prime}\right)$-phase transition while smaller $(<5.4 \mathrm{~nm})$ one do not show an $\left(\alpha-\alpha^{\prime}\right)$-phase transition.

- A structure transition in the 5.0 and the $4.8 \mathrm{~nm}$ clusters from icosahedral to cubic was observed after hydrogen loading. This transition was found to be reversible in the 4.8 $\mathrm{nm}$ cluster and irreversible in the $5.0 \mathrm{~nm}$ cluster. Smaller clusters 3.6 and $3.0 \mathrm{~nm}$ maintain their icosahedral structure during hydrogen loading.

- Plotting the lattice parameter of different Pd cluster sizes as a function of the hydrogen pressure show that there is a narrowing in the lattice parameter miscibility gap as the cluster size decreases. For small clusters a linear increase of the lattice parameter values with hydrogen absorption is found, while the lattice expands strongly for large clusters in a narrow pressure range (constant chemical potential).

- Pressure-lattice parameter isotherms show the existence of a hysteresis during hydrogen desorption even for small Pd clusters. 
- Gravimetric measurements show that:

- Pressure-concentration isotherms of all clusters show an increase in the $\alpha$-phase solubility in comparison to bulk Pd. This is attributed to the additional absorption in the surface and subsurface sites.

- The absorption isotherm for Pd-powder shows a slope of 2.0 at low concentration as expected from Sieverts' law. The slope for the Pd cluster samples is: 1.6 for the 5.18 $\mathrm{nm}$ cluster, 2.0 for the $4.8 \mathrm{~nm}$ cluster 1.3 for the $3.6 \mathrm{~nm}$ cluster and 2.2 for the $3.0 \mathrm{~nm}$ cluster. Slope values lower than 2 can be attributed to $\mathrm{H}-\mathrm{H}$ interaction.

- The width of the plateau (miscibility gap) of the Pd-H phase boundary exhibits significant narrowing with decreasing cluster size. This is explained by the reduced number of bulk like sites in clusters whereby the surface and subsurface sites do not participate in the phase transition.

- The pressure-concentration isotherms of all clusters show a slope in the plateau pressure which do not usually occurs in bulk Pd. The slope can be attributed to a possible adhesion tension between the surfactant and the cluster.

- All desorption isotherms show a hysteresis effect. This verifies the existence of a twophase field even in small clusters. The hysteresis can be explained by the thermodynamic of an open coherent two-phase system. 


\section{Appendix A}

\section{A.1 The electrolysis parameter study}

A detailed study concerning the size-selective electrochemical preparation of tetraoctylammonium-stabilised palladium clusters is performed. Different electrolysis parameters such as current density $\left(I_{d}\right)$, distance between electrodes $\left(D_{E}\right)$, temperature $(T)$, and solvent polarity (S) were studied.

The study of each of these parameters is discussed below in detail:

\section{A.1.1 Electrolysis at different current density}

The effect of the applied electrolysis current density $\left(\mathrm{I}_{\mathrm{d}}\right)$ was studied. In each experiment different constant currents (50 mA, $25 \mathrm{~mA}, 13 \mathrm{~mA}, 5 \mathrm{~mA}$ and $1 \mathrm{~mA}$ ) were applied to the cell, while the other electrolysis parameter were kept constant. The current density $\left(I_{d}\right)$ was then calculated taken into account the effective electrode area.

The electrolysis parameters used in this study are summarised in Table A.1.

Table A. 1. Electrolysis at different current parameters

\begin{tabular}{l|lll}
\hline$I[\mathrm{~mA}]$ & $I_{d}\left[\mathrm{~mA} / \mathrm{cm}^{2}\right]$ & Pd dissolved [mg] & Anodic efficiency [\%] \\
\hline 50 & 5.71 & 308 & 77.6 \\
25 & 2.86 & 349 & 87.9 \\
13 & 1.49 & 214 & 53.9 \\
5 & 0.57 & 333 & 83.9 \\
1 & 0.11 & & \\
\hline
\end{tabular}

Reaction conditions: $\mathrm{T}=22^{\circ} \mathrm{C}, \mathrm{D}_{\mathrm{E}}=3 \mathrm{~mm}$, electrolyte: $(90 \mathrm{ml}) 0.1 \mathrm{M} \mathrm{Oc}_{4} \mathrm{~N}^{+} \mathrm{Br}^{-}$in $\mathrm{CH}_{3} \mathrm{CN}$ and $\mathrm{Q}=720 \mathrm{~A} \cdot \mathrm{s}$ (except in the electrolysis at $\mathrm{I}_{\mathrm{d}}=0.11 \mathrm{~mA} / \mathrm{cm}^{2}$ ). 


\section{A.1.2 Electrolysis at different temperatures}

The effect of temperature on the size of the palladium cluster was studied. In a typical series of experiments electrolyses were performed at $60^{\circ} \mathrm{C}, 40{ }^{\circ} \mathrm{C}, 22^{\circ} \mathrm{C}$ and $16^{\circ} \mathrm{C}$. The temperature was held constant using a thermostatic water bath. Table A. 2 summarises the parameters used in this study.

Table A. 2. electrolysis at different temperatures

\begin{tabular}{c|cc}
\hline$T\left[{ }^{\circ} \mathrm{C}\right]$ & Pd dissolved [mg] & Anode efficiency [\%] \\
\hline 60 & 276 & 70.0 \\
40 & 273 & 68.8 \\
22 & 349 & 87.9 \\
16 & 289 & 72.8 \\
\hline
\end{tabular}

Reaction conditions: $\overline{\mathrm{D}_{\mathrm{E}}=3 \mathrm{~mm}, \mathrm{I}_{\mathrm{d}}=2.86 \mathrm{~mA} / \mathrm{cm}^{2} \text {, electrolyte: }(90 \mathrm{ml}) 0.1 \mathrm{M} \mathrm{Oc}_{4}} \mathrm{~N}^{+} \mathrm{Br}^{-}$in $\mathrm{CH}_{3} \mathrm{CN}, \mathrm{Q}=$ $720 \mathrm{~A} \cdot \mathrm{s}$

\section{A.1.3 The distance between electrodes $\left(D_{E}\right)$}

In four series of experiments the anode and the cathode, both $\mathrm{Pd}$, were placed $10 \mathrm{~mm}, 5 \mathrm{~mm}$, $3 \mathrm{~mm}$ and $1 \mathrm{~mm}$ from one another, Table A.3. Applying a current density of $2.86 \mathrm{~mA} / \mathrm{cm}^{2}$ while keeping the other electrolyses parameter constant the effect of $\mathrm{D}_{\mathrm{E}}$ on the cluster size was studied.

Table A. 3. Electrolyses at different electrode distance.

\begin{tabular}{c|cc}
\hline$D_{E}[\mathrm{~mm}]$ & Pd dissolved [mg] & Anode efficiency [\%] \\
\hline 10 & 279 & 70.3 \\
5 & 257 & 64.5 \\
3 & 349 & 87.9 \\
1 & 263 & 66.7
\end{tabular}

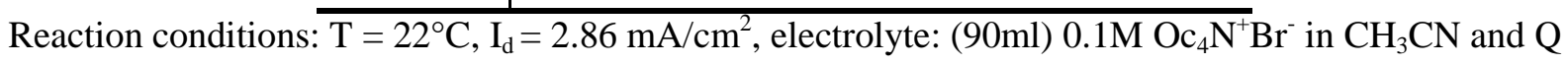
$=720 \mathrm{~A} \cdot \mathrm{s}$ 


\section{A.1.4 Electrolysis in different solvent}

A separate study to probe the influence of the solvent polarity on the cluster size was conducted using $\mathrm{CH}_{3} \mathrm{CN} / \mathrm{THF}$ mixtures of precisely defined composition in which the percentage of $\mathrm{CH}_{3} \mathrm{CN}$ varied from $100 \%$ to $0 \%$. All other parameters were kept constant, Table A.4.

Table A. 4. Electrolyses in different solvent polarity

\begin{tabular}{c|cc}
\hline $\begin{array}{c}\text { solvent } \\
\mathrm{CH}_{3} \text { CN:THF }\end{array}$ & Pd dissolved [mg] & Anode efficiency [\%] \\
\hline $1: 0$ & 349 & 87.9 \\
$1: 1$ & 310 & 78.1 \\
$1: 2$ & 298 & 75.1 \\
$1: 3$ & 296 & 74.6 \\
$1: 4$ & 274 & 69.0 \\
$0: 1$ & 209 & 52.6 \\
\hline
\end{tabular}

Reaction conditions: $\mathrm{T}=22^{\circ} \mathrm{C}, \mathrm{D}_{\mathrm{E}}=3 \mathrm{~mm}, \mathrm{I}_{\mathrm{d}}=2.86 \mathrm{~mA} / \mathrm{cm}^{2}$, electrolyte: $(90 \mathrm{ml}) 0.1 \mathrm{M} \mathrm{Oc}_{4} \mathrm{~N}^{+} \mathrm{Br}^{-}$in one of the above solvents, $\mathrm{Q}=720 \mathrm{~A} \cdot \mathrm{s}$ 


\section{References}

[1] http://www.psc.edu/science/Wolf/Wolf.html

[2] T. Graham, Phil. Trans. Roy. Soc. London, 156 (1866) 399

[3] S. Sugano, H. Koizumi, in: Microcluster Physics, Springer, Berlin, Heidelberg, 1998

[4] T. Mütschele and R. Kirchheim, Scripta Metallurgica, 21(1987)1101

[5] H. Natter, B. Wettmann, B. Heisel, R. Hempelmann, J. Alloys Comp., 253 (1997) 84

[6] U. Stuhr, H. Wipf, T. J. Udovic, J. Weissmüller, H. Gleiter, J. Phys. Cond. Matter, 7 (1995) 219

[7] A. Pundt, C. Sachs, M. Winter, M. T. Reetz, D. Fritsch, R. Kirchheim, J. Alloys Comp., 293-295 (1999) 480

[8] C. Sachs, A. Pundt, R. Kirchheim, M. T. Reetz, D. Fritsch, Phys. Rev. B, 64 (2001) 75408

[9] C. Nützenadel, A. Züttel, D. Chartouni, G. Schmid, L. Schlapbach, Eur. Phys. J., D 8 (2000) 245

[10] A. Züttel, Ch. Nützenadel, G. Schmid, Ch. Emmenegger, P. Sudan, L. Schlapbach, Applied Surface Science, 162-163 (2000) 571

[11] A. Züttel, Ch. Nützenadel, G. Schmid, D. Chartouni, L. Schlapbach, J. Alloy. Comp., 293-295 (1999) 472

[12] G. Schmid (Ed.), Clusters and Colloids, VCH Weinheim 1994.

[13] N.A. Shipway, E. Katz and Willner, CHEMPHYSCHEM, 1 (2000) 18

[14] S. Chen, R- S. Ingram, M. J. Hostetler, J. J. Pietron, R. W. Murray, T. G. Schaaff, J. T. Khoury, M. M. Alvarez, R. L. Whetten, SCIENCE, 280 (1998).2098

[15] D. G: Kurth, P. Lehmann and C. Lesser, Chem. Commun.,949-950, 2000

[16] R. Elghanian, J. J. Storhoff, R. C. Mucic, R. L. Letsinger, C. A. Mirkin, SCIENCE, 277 (1997) 1078

[17] L. N: Lewis, Chem. Rev., 93 (1993) 2693

[18] Z. Xiaobin, L. Hanfan and L. Manhong, Tetrahedron Letters, 34(14) (1998) 1941

[19] G. Schmid, S. Emde, V. Maihack, W. Meyer-Zaika, St. Peschel, J. Molecular Catalysis

A: Chemical 107 (1996) 95

[20] K. Kurihara, J.Kizling, P. Stenius, J. H. Fendler, J. Am. Chem. Soc., 105 (1983) 2574

[21] E. Salomoms, R. Griessen, D.G: de Groot, A. Magerl, Europhys. Lett., 5 (1988) 449 
[22] L. D. Marks, Rep. Prog. Phys., 57 (1994) 603

[23] U. Laudahn, A. Pundt, M. Bicker . U. Hülsen, U. Geyer, R. Kirchheim, J. Alloys Comp., 490 (1999) 293

[24] A. Sieverts, Z. Physik. Chem., 88 (1914) 451

[25] H. Frieske und E. Wicke, Ber. Bunsenges. Physik. Chem., 77 (1973) 48

[26] F. A. Lewis, The Palladium Hydrogen System, Academic Press, 1967

[27] T. Fliessbach, Statistische Physik, Spektrum Akademischer Verlag GmbH, Heidelberg Berlin Oxford (1995)

[28] Y. Fukai, The Metal-Hydrogen System, Springer-Verlag1993

[29] C. Wagner, Z. Physik. Chem. A., 193(386) (1994) 407

[30] H. Brodowsky, Z. Physik. Chem. NF, 44 (1965) 129

[31] G. Alefeld, Phys. Stat. Sol., 32 (1969) 67

[32] P. Haasen, Physical Metallurgy, Cambridge University Press, third edition (1996)

[33] E. Wicke and Brodowsky, Hydrogen in Metals II, volume 29 Topics in Applied physics, Chapter 3 Springer-Verlag, Berlin Heidelberg (1978), Eds. G. Alefeld and J. Völkl.

[34] E. Wicke and G. H. Nenst, Ber. Bunsenges. Phys. Chem., 68 (1964) 224

[35] T. Graham, Phil. Trans. Roy. Soc. London, 156 (1866) 399

[36] T. Mütschele and R. Kirchheim, Scripta Metallurgica, 21 (1987) 135

[37] T. B. Flanagan, B. S. Bowerman, G. E. Biehl, Scripta Met., 14 (1980) 443

[38] B. J. Makenas, H. K. Birnbaum, Acta Met., 28 (1980) 979

[39] G. Sauthoff, Z. Metallkde, 67 (1976) 25

[40] J. W. Cahn, F. Larche', Acta Met., 32 (1984) 1915

[41] R. B. Schwarz, A. G. Khachaturyan, Phys. Rev. Lett., 72: (1995) 2523

[42] A. G. Khachaturyan, The Theory of Structural Transformation in Solids, Wiely \& Sons, New York, 1983

[43] L. Schlapbach in: Hydrogen in Intermetallic Compopunds II, Editor L. Schlapbach, Springer-Verlag, 1992

[44] K. Christmann, Molecular Phys., 66 (1989) 1

[45] M. Strongin, J. Colbert, G. J. Dienes D. O. Welch, Phys. Rev. B.,26 (1982) 2715

[46] M. A. Pick, A. Hanson, K. W. Jones, A. N. Goland, Phys. Rev. B, 26 (1982) 2900

[47] E. Wicke, Z. Phys. Chem. NF, 143 (1985) 1

[48] R. Kirchheim. Physica Scripta., T94 (2001) 58 
[49] H. Haberland (Ed.), Clusters of atoms and molecules, Springer-Verlag Berlin Heidelberg New York 1994

[50] J. S. Bradly, in: Clusters and Colloids, (Ed.: G. Schmid) VCH Weinheim, 1994, p477f.

[51] T. Graham, Phil. Trans. Roy. Soc. (London) 154 (1899) 415

[52] S. Sugano and H. Koizumi, Microcluster physics-p7]

[53] M. M. Kappes, Chem. Rev. 88 (1988) 369

[54] R. L. Johnston, Phli. Trans R. Soc. Lond A. 356 (1735) (1998) 211

[55] A. L. Mackay Acta Cryst., 15 (1962) 916

[56] T. P. Martin, Physics Reports, 273 (1996) 199

[57] D. G. Duff, A. C. Curtis, P. P. Edwards. D. A. Jefferson, B. F. G. Johanson and D. E. Logan, J. chem. Soc. Chem. Commun. 1264, 1987

[58] M. E. Libab. Colloids and surfaces 29 (1988) 293

[59] T. Teranishi, M. Hosoe, T. Tanaka, M. Miyake, J. Phys. Chem., 103 (1999) 3818

[60] T. S. Ahmai, Z. L. Wang, T. C. Henglein, M. A. El-Sayed, Science, 272 (1996) 1924

[61] J. Turkevich, P. C. Stevenson, J. Hiller, Discuss. Farady Soc., 11 (1951) 55

[62] Y. Wang, J. Ren, K. Deng, L. Gui and Y. Tang, Chem. Mater, 12 (2000) 1622

[63] T. Teranishi and M. Miyake, Chem. Mater, 10 (1998) 594

[64] M. T: Reetz, W. Helbig, J. Am. Chem. Soc., 116 (1994) 7401

[65] M. Faraday, Philo. Trans. R. Soc. London, 147 (1857) 145

[66] J. D. AikenIII, R. G. Finke, J. Mol. Catal. A, 145 (1999) 1

[67] H. Bönnemann and R. M. Richards, Eur. J. Inorg. Chem. 2455, 2001

[68] B. G. Ershov, E. Janata, M: Michaelis, A. Henglein, J. Phys. Chem., 95 (1991) 8996

[69] J. S.,Bradly, E.W. Hill, S. Behal, C. Klein, B. Chaudert, A. Duteil, Chem. Mater, 4 (1992) 1234

[70] K. J. Klabunde, G. Youngers, E. J. Zuckerman, B. J. Tan, S. Antrim, P. M. Sherwood, Eur. J. Solid State Inorg. Chem., 29 (1992) 227

[71] N. Ibl, Chem. Ing. Tech., 36 (1964) 601-612

[72] S. C. Davis, K. J. Kalbunde, Chem. Rev. 82 (2) (1982) 153

[73] Southhampton Electrochemistry Group, Instrumental Methods in Electrochemistry, Ellis Horwood Ltd., 1990 chapter 9

[74] W. Helbig, Dissertation Ruhr-University Bochum 1994

[75] M. T. Reetz, M. Winter, R. Breinbauer, T. Th. Albrecht, W. Vogel, Chem. Eur. J. 7(5) (2001) 1084 
[76] C Reichardt, Solvents and Solvents effects in Organic Chemistry, 2nd ed., VCH, Weinheim, 1988

[77] M. T. Reetz, W. Helbig, S.A. Quaiser, U. Stimming, N. Breuer, R. Vogel, Science, 267 (1995) 367

[78] B. D. Hall, D. Zanchet, D. Ugarte, J. Appl. Cryst., 33 (2000) 1335

[79] C. W. B. Grigson and E. Barton, Brit. J. Appl. Phys., 18 (1967) 175-183

[80] B. D. Hall, J. Appl. Phys., 87 (2000) 1666-1675

[81] A. Züttel, Ch. Nützenadel, G. Schmid, D. Chartouni, L. Schlapbach, J. Alloys Comp., 293-295 (1999) 472-475

[82] J. W. Lee, G. D. Stein, J. Chem. Phys., 91 (1987) 2450

[83] N. M. Jisrawi, A. Pundt, M. Guerdane, H. Teichler, submitted to J. Phys. Chem.

[84] A. Pundt, M. Suleiman, R. Kirchheim, submitted to Materials Science and Engineering B

[85] M. Suleiman, N. M: Jisrawi, O. Dankert, M. T. Reetz, C. Bähtz, R. Kirchheim, A. pundt, J. Alloy. Comp., Article in press

[86] H. Okuyama, W. Siga, N. Takagi, M. Nishijima, T. Aruga, Surf. Sci., 401 (1998) 344

[87] V. Srivastava and R. Balasubramaniam, Material Science and engineering, A 304-306 (2001) 897

[88] E. Salomoms, R. Griessen, D.G: de Groot, A. Magerl, Europhys. Lett., 5 (1988) 449

[89] A. Pundt Postdoctoral Thesis University of Göttingen 2002

[90] X. Fu, Y Wang, N. Wu, L. Gui and Y. Tang, J. of Colloid and Interface Science, 243 (2001) 326 


\section{Danksagung}

Herrn Prof. Dr. R. Kirchheim danke ich, dass er es mir ermöglicht hat, meine Doktorarbeit am Institut für Materialphysik durchzuführen. Vielen Dank für die vielseitige und fruchtbare Themenstellung, und auch für die vielen anregenden und konstruktiven Diskussionen.

Besonderer Dank gilt meiner Betreuerin Astrid Pundt, die durch ihr beständiges Interesse und wertvolle Diskussionen immer wieder zum Gelingen dieser Arbeit beitrug. Vielen Dank auch für ihr andauerndes Bemühen und die ausgezeichnete Arbeitsatmosphäre.

Den weiteren Mitgliedern unserer Arbeitsgruppe, auch den ehemaligen: Olof Dankert, Martin Dornheim, Joachim Bankmann, Eugen Nikitin, Kai Nörthemann und Diana Marcano danke ich für viele anregende und spannende Gruppensitzungen und Messreisen.

Mein besonderer Dank gilt Christine Borchers für die Zusammenarbeit und die Durchführung von TEM Messungen.

Herrn Prof. Dr. H. S. Hilal danke ich für die Unterstützung wahrend meiner Doktorarbeit.

Herrn Prof. Dr. M. Reetz vom Max-Plank-Institut für Kohlenforschung danke ich für die fortlaufende Kooperation auf dem Gebiet der Cluster und die Gastfreundschaft am Institut.

Für ihre wertvolle Hilfe beim Korrekturlesen dieser Arbeit bedanke ich mich sehr herzlich bei N. Jisrawi, T. Al Kassab und J. Bankmann.

Meinem treuen Freund Hamid Guerdane möchte ich für seine Freundschaft und sein Verständnis sehr danken. Mit ihm konnte ich immer über das Leben und die Welt philosophieren. Imad Ladadwa möchte ich für die Freundschaft und die gemeinsame Zeit danken.

Allen Helfern in der Feinmechanik, der E- Werkstatt und den Physiklaboranten gilt mein Dank. Besonders sei Karin Ahlborn und Matthias Hahn gedankt.

Dem Deutschen Akademischen Austauschdienst (DAAD) danke ich für das mir gewährte Promotionsstipendium.

Meinen Eltern möchte ich für die vorbehaltlose Unterstützung in allen Lebenslangen danken. Mein besonderer Dank geht an meine Frau und meinen Sohn, die mit ihrem Verständnis, außergewöhnlicher Geduld und ihrer Liebe dies alles erst ermöglicht haben. 


\section{Lebenslauf}

\section{Persönliche Daten}

Name:

Mohammed A. M. Shtaya-Suleiman

Geburtsdatum:

Geburtsort:

15.11.1966

Familienstand:

Nationalität:

Salfeet West Bank

verheiratet, $1 \mathrm{Kind}$

Palästinenser.

\section{Ausbildung}

1973-1979

Grundschule: Salfeet Elementary school

1979-1986

Gymnasium: Salfeet high school

\section{Studium}

1986-1992 Chemiestudium an An-Najah N. University Nablus.

1992

BSc in Chemie.

1995

MSc in Chemie

seit 08/1999 Wissenschaftlicher Mitarbeiter am Institut für Materialphysik an der GeorgAugust-Universität Göttingen

\section{Berufstätigkeit}

1992-1993 Palestine Pharmaceutical Co. quality control manager

1993-1994 Wissenschaftlicher Mitarbeiter an der An-Najeh N. University

1995-1997 Chemielehrer: Salfeet Secondary High School.

1997-1998 Curriculum Center PNA

Coordinator of science team. 